\title{
THE COMPONENTS OF THE AUTOMORPHISM GROUP OF A JORDAN ALGEBRA $\left({ }^{1}\right)$
}

\author{
BY

\section{S. ROBERT GORDON}

\begin{abstract}
Let $\Im$ be a semisimple Jordan algebra over an algebraically closed field $\Phi$ of characteristic zero. Let $G$ be the automorphism group of $\mathfrak{\Im}$ and $\Gamma$ the structure groups of $\Im$. General results on $G$ and $\Gamma$ are given, the proofs of which do not involve the use of the classification theory of simple Jordan algebras over $\Phi$. Specifically, the algebraic components of the linear algebraic groups $G$ and $\Gamma$ are determined, and a formula for the number of components in each case is given. In the course of this investigation, certain Lie algebras and root spaces associated with $\Im$ are studied.

For each component $G_{i}$ of $G$, the index of $G$ is defined to be the minimum dimension of the 1-eigenspace of the automorphisms belonging to $G_{i}$. It is shown that the index of $G_{i}$ is also the minimum dimension of the fixed-point spaces of automorphisms in $G_{i}$. An element of $G$ is called regular if the dimension of its 1-eigenspace is equal to the index of the component to which it belongs. It is proven that an automorphism is regular if and only if its 1-eigenspace is an associative subalgebra of $\mathfrak{~}$. A formula for the index of each component $G_{i}$ is given.

In the Appendix, a new proof is given of the fact that the set of primitive idempotents of a simple Jordan algebra over $\Phi$ is an irreducible algebraic set.
\end{abstract}

Introduction. Let $\mathfrak{\Im}$ be a semisimple Jordan algebra over an algebraically closed

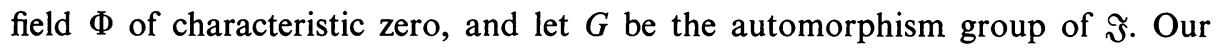
purpose in the following is to present general results on $G$ which, are analogous to certain well-known results on Lie algebras, the proofs of which do not involve the use of the classification theory of simple Jordan algebras over $\Phi$. Specifically, we wish to determine the algebraic components of the linear algebraic group $G$; that is, if $G_{0}$ is the component of the identity of $G$, we wish to find the cosets $G_{0}, G_{1}$, $G_{2}, \ldots$ of $G_{0}$. For each component $G_{i}$ of $G$ we will investigate the following question: For $\eta \in G_{i}$, how small can the dimension of the fixed-point space of $\eta$ be ? Finally, we will apply the results of this investigation to each of the simple Jordan algebras over $\Phi$.

Received by the editors December 1, 1969.

AMS 1969 subject classifications. Primary 1740, 1730; Secondary 2070, 1450.

Key words and phrases. Semisimple Jordan algebra, characteristic zero, automorphism group, algebraic components, fixed points, derivation algebra, Lie algebra, Koecher-Tits algebra, root space.

( ${ }^{1}$ The results in this paper are contained in the author's doctoral thesis, written at Yale University under the direction of Professor N. Jacobson. They were previously announced in [3]. The author would like to express his gratitude to Professor Jacobson and also to Professor G. B. Seligman for their assistance. The research was supported in part by an NSF Graduate Fellowship and in part by NSF Grant GP-8023.

Copyright (C) 1971, American Mathematical Society 
The corresponding questions for Lie algebras are answered in Chapter 9 of [8] and in [9], the contents of which we summarize here. Let $\mathfrak{L}$ be a semisimple Lie algebra over an algebraically closed field of characteristic zero, and let $G=$ Aut $\mathfrak{L}$, the automorphism group of $\mathfrak{L}$. Then there is a natural isomorphism between the group $G / G_{0}$ of algebraic components of $G$ and the group of automorphisms of the Dynkin diagram of $\mathfrak{R}$, which is described as follows: Let $\mathfrak{H}$ be a Cartan subalgebra of $\mathfrak{L}$, and let $\eta \in G$. Then there exists an automorphism $\tau \in G_{0}$, the component of the identity of $G$, such that $\eta \tau$ stabilizes $\mathfrak{S}$. The dual transformation $(\eta \tau)^{*}$ of $\eta \tau \mid \mathfrak{S}$ permutes the roots of $\mathfrak{Q}$ and so is of the form $w_{1} w_{2}$, where $w_{1}$ is in the Weyl group of $\mathfrak{L}$ and $w_{2}$ stabilizes a simple system of roots of $\mathfrak{L}$. Thus $w_{2}$ induces an automorphism $\pi$ of the diagram of $\mathfrak{L} ; \pi^{-1}$ is the automorphism corresponding to the component $\eta G_{0}$ of $\eta$. If $G_{i}$ is a component of $G$ we define the index of $G_{i}$ to be the minimum dimension of the 1-eigenspaces of automorphisms belonging to $G_{i}$; we call an element $\eta$ of $G_{i}$ regular if the dimension of its 1-eigenspace is the index of $G_{i}$. In [9] it is shown that the 1-eigenspace of any automorphism $\eta$ of $\mathfrak{Q}$ is a reductive subalgebra of $\mathfrak{Q}$ which is abelian if and only if $\eta$ is regular, and that the index of a component $G_{i}$ is the number of orbits of the corresponding automorphism of the Dynkin diagram of $\mathfrak{L}$ (regarded as a permutation of the points of the diagram). It is also shown that the index of $G_{i}$ is the minimum dimension of the fixed-point spaces of automorphisms belonging to $G_{i}$.

Our method in studying the Jordan algebra $\mathfrak{\Im}$ and its automorphism group will be to transfer the problems to certain Lie algebras associated with $\mathfrak{\Im}$. Three Lie

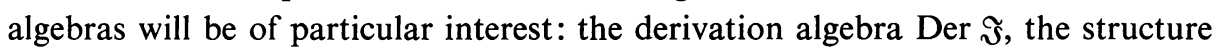
Lie algebra $\mathfrak{L}(\mathfrak{\Im})$, and the Koecher-Tits algebra $\mathfrak{I}(\mathfrak{\Im})$. The reader is referred to Chapter 8 of [10] for the definitions and elementary properties of these algebras.

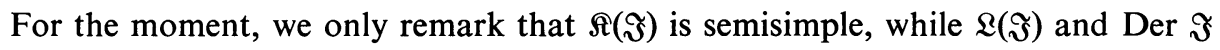
are completely reducible, so that their derived algebras $\mathfrak{Q}(\mathfrak{\Im})^{\prime}$ and Der $\mathfrak{S}^{\prime}$ are semisimple. We shall study in particular the root spaces and Weyl groups of Der $\mathfrak{\Im}^{\prime}$, $\mathfrak{L}(\Im)^{\prime}$, and $\mathfrak{I}(\Im)$, so that we may make use of the results on automorphisms of Lie algebras summarized above. Finally, we shall occasionally need to use facts about linear algebraic groups, for which we offer [2] as a suitable reference.

1. Preliminaries. In the following, except when explicitly stated otherwise, $\mathfrak{\Im}$ will be a finite-dimensional semisimple Jordan algebra over an algebraically closed field $\Phi$ of characteristic zero. The product of two elements $x, y \in \Im$ will be written

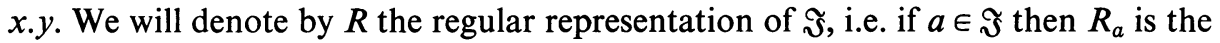
mapping $x \mapsto x . a$ of $\Im$ into itself. We will denote by $U$ the quadratic representation

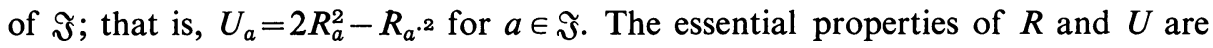
given in Chapter 1 of [10]; for the moment we mention only the so-called "Fundamental Formula" of Jordan algebras, namely

$$
U_{a} U_{b} U_{a}=U_{b U_{a}}
$$

for all $a, b \in \Im$, and the fact that $a \in \mathfrak{\Im}$ is invertible if and only if $U_{a}$ is invertible. 
Let $G$ be the automorphism group and $\Gamma$ the structure group of $\Im$. Here $\Gamma$ is defined to be the set of nonsingular linear transformations $\eta$ of $\mathfrak{\Im}$ into itself such that there exists a nonsingular linear transformation $\eta^{\#}$ of $\Im$ satisfying $\eta^{\#} U_{a} \eta=U_{a \eta}$ for every $a \in \mathfrak{~}$. Here $\eta^{\#}$ is uniquely determined, for if we take $a=1$, we find that $\eta^{\#}=U_{1 \eta} \eta^{-1}$. It is clear that $G \subseteq \Gamma$, since if $\eta \in G$ then $\eta^{-1} U_{a} \eta=U_{a \eta}$ for all $a \in \mathfrak{\Im}$. The Fundamental Formula implies that $U_{a} \in \Gamma$ for all invertible elements $a$ of $\Im$. On page 59 of [10] it is shown that $G=\{\eta \in \Gamma \mid 1 \eta=1\}$. Both $G$ and $\Gamma$ are linear algebraic groups; this fact is well known for $G$, and follows for $\Gamma$ from the observation that the conditions $U_{1 \eta} \eta^{-1} U_{a} \eta=U_{a \eta}$ for all $a \in \Im$ are expressible as a set of polynomial equations on $\operatorname{Hom}_{\Phi}(\Im, \Im)$. We will denote by $G_{0}$ and $\Gamma_{0}$ the algebraic components of the identity of the groups $G$ and $\Gamma$, respectively, and in general for any linear group $L, L_{0}$ will denote the component of the identity of $L$.

We will be concerned with several Lie algebras associated with $\mathfrak{\Im}$. $\mathfrak{D}=$ Der $\mathfrak{T}$ will be the derivation algebra of $\mathfrak{\Im}$. We recall that linear transformations of $\mathfrak{\Im}$ of the form $\left[R_{a}, R_{b}\right], a, b \in \mathfrak{\Im}$, are derivations of $\mathfrak{\Im}$; sums of such derivations are called inner derivations [10, p. 35]. Since $\Im$ is semisimple and the characteristic of $\Phi$ is zero, every derivation of $\mathfrak{I}$ is inner, i.e. $\mathfrak{D}=\left[R_{\mathfrak{S}}, R_{\mathfrak{J}}\right]$. Also $\mathfrak{D}$ is a completely reducible Lie algebra of linear transformations of $\mathfrak{F}$, so that $\mathfrak{D}=\mathfrak{E} \oplus \mathfrak{D}^{\prime}$, where $\mathfrak{E}$ is the center of $\mathfrak{D}$, and $\mathfrak{D}^{\prime}$, the derived algebra of $\mathfrak{D}$, is semisimple (for proofs of these facts about $\mathfrak{D}$, see [10, pp. 323-324]). If $\mathfrak{\Im}=\mathfrak{I}_{1} \oplus \mathfrak{\Im}_{2}$, where $\mathfrak{\Im}_{1}$ and $\mathfrak{\Im}_{2}$ are ideals of $\mathfrak{T}$, then $\mathfrak{D} \cong \operatorname{Der} \mathfrak{\Im}_{1} \oplus$ Der $\mathfrak{\Im}_{2}$, where the isomorphism identifies Der $\mathfrak{\Im}_{1}$ with $\left\{D \in \mathfrak{D} \mid \Im_{2} D=0\right\}$ and Der $\Im_{2}$ with $\left\{D \in \mathfrak{D} \mid \Im_{1} D=0\right\}$.

By $\mathfrak{L}=\mathfrak{L}(\mathfrak{)})$ we will mean the structure Lie algebra $R_{\mathfrak{S}} \oplus \mathfrak{D}$ of $\mathfrak{\Im}$. This is a Lie algebra since $\left[R_{\mathfrak{S}}, R_{\mathfrak{S}}\right] \subseteq \mathfrak{D}$ and since for $a \in \mathfrak{F}, D \in \mathscr{D}$, we have $\left[R_{a}, D\right]=R_{a D}$. It is also a completely reducible algebra of linear transformations of $\mathfrak{\Im}$; in fact, the simple ideals of $\mathfrak{\Im}$ are the irreducible $\mathfrak{L}$-submodules of $\mathfrak{\Im}$. Hence $\mathfrak{L}=\mathfrak{C} \oplus \mathfrak{Q}^{\prime}$, where $\mathfrak{C}$ is the center of $\mathfrak{L}$ and $\mathfrak{L}^{\prime}$, the derived algebra of $\mathfrak{L}$, is semisimple. For $x, y$, $z \in \mathfrak{F}$ we define the associator of $x, y$, and $z$ to be $[x, y, z]=(x . y) . z-x .(y . z)$; by $\mathfrak{I}^{\prime}$ we mean the subspace of $\mathfrak{\Im}$ spanned by all associators. Then $\mathfrak{L}^{\prime}=R_{\mathfrak{Y}} \oplus \mathfrak{D}[10$, proof of Theorem 3, p. 314]. It is also clear that $\mathfrak{L}\left(\mathfrak{J}_{1} \oplus \mathfrak{\Im}_{2}\right) \cong \mathfrak{L}\left(\mathfrak{\Im}_{1}\right) \oplus \mathfrak{L}\left(\mathfrak{\Im}_{2}\right)$ in a natural way, since $\Im_{1} \cdot \Im_{2}=0$.

By $\mathfrak{R}=\mathfrak{\Re}(\Im)$ we will mean the Koecher-Tits algebra of $\mathfrak{~}$. This algebra has as the underlying vector space $\Im \oplus \Im \oplus \mathfrak{Q}$, the direct sum of $\mathfrak{L}$ and two copies of $\Im$. The product in $\mathfrak{R}$ is given by anticommutativity and the following:

$\mathfrak{Q}$ is a subalgebra of $\mathfrak{R}$,

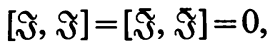

$\left[x, R_{a}+D\right]=x\left(R_{a}+D\right)$ and $\left[\bar{x}, R_{a}+D\right]=\left(x\left(-R_{a}+D\right)\right)^{-}$if $x, y \in \mathfrak{J}, D \in \mathscr{D}$, $[x, \bar{y}]=2\left(R_{x . y}-\left[R_{x}, R_{y}\right]\right)$ if $x, y \in \Im$.

(Typographical convenience has throughout this paper required the occasional use of the awkward notations $(A)^{-},(A)^{\sim},(A)^{\wedge}$ in place of $\bar{A}, \tilde{A}, \hat{A}$, respectively, whenever $A$ is a sequence of two or more symbols.) In this definition we follow Koecher [11] rather than Jacobson [10], who defines $[x, \bar{y}]$ to be $R_{x, y}-\left[R_{x}, R_{y}\right]$. 
It is easy to check that the two definitions yield isomorphic algebras. That $\Re$ is a Lie algebra with this product is proven in [10, pp. 325-326], or in [11, p. 799]. $\Omega$ is semisimple if and only if $\mathfrak{\Im}$ is semisimple, and is simple if and only if $\mathfrak{\Im}$ is simple [10, pp. 332-333] (or [11, pp. 794 and 805]). As before, $\mathfrak{A}\left(\Im_{1} \oplus \mathfrak{\Im}_{2}\right) \cong \Re\left(\Im_{1}\right)$ $\oplus \mathfrak{I}\left(\Im_{2}\right)$. By $\varepsilon$ we will mean the automorphism of $\mathfrak{\Re}$ which sends $a+\bar{b}+R_{c}+D$ $\rightarrow b+\bar{a}-R_{c}+D$ for all $a, b, c \in \Im$ and $D \in \mathscr{D}$. We will also use the same letter $\varepsilon$ to denote the restriction of this mapping to $\mathfrak{R}$, i.e. the automorphism of $\mathfrak{L}$ given by $R_{a}+D \mapsto-R_{a}+D$ for all $a \in \Im, D \in \mathfrak{D}$.

We will be interested in Cartan subalgebras of these Lie algebras. In this connection we have the following general

Lemma. Let $\mathfrak{Q}$ be a Lie algebra and $\mathfrak{\mathfrak { C }}$ an abelian Lie algebra. If $\mathfrak{S}_{1}$ is a Cartan subalgebra of $\mathfrak{L}$, then $\mathfrak{S}=\mathfrak{C} \oplus \mathfrak{F}_{1}$ is a Cartan subalgebra of $\mathbb{C} \oplus \mathfrak{L}$. Conversely, if $\mathfrak{S}$ is a Cartan subalgebra of $\mathfrak{S} \oplus \mathfrak{Q}$, then $\mathfrak{S}_{2}=\mathfrak{C} \oplus \mathfrak{S}_{1}$, where $\mathfrak{S}_{1}=\mathfrak{S}_{\mathcal{L}} \cap \mathfrak{L}$ is a Cartan subalgebra of $\mathbf{Q}$.

Proof. Since $\mathfrak{S}_{1}$ is nilpotent and $\left[\mathfrak{S}, \mathfrak{S}_{\mathfrak{Q}}\right] \subseteq[\mathfrak{C}, \mathfrak{C}]+\left[\mathfrak{C}, \mathfrak{S}_{1}\right]+\left[\mathfrak{S}_{1}, \mathfrak{S}_{1}\right]=0+0$ $+\left[\mathfrak{S}_{1}, \mathfrak{S}_{1}\right], \mathfrak{S}$ is also nilpotent. If $c \in \mathfrak{V}, x \in \mathfrak{L}$ are such that $\operatorname{ad} \mathfrak{s} \oplus \mathfrak{Q}(c+x)$ normalizes $\mathfrak{S}_{2}$, then ad $x$ normalizes $\mathfrak{S}_{1}$. I.e., $x \in \mathfrak{S}_{1}$. Hence $\mathfrak{C} \oplus \mathfrak{S}_{1}$ is a Cartan subalgebra of (5) $\oplus \mathfrak{R}$.

Let $\mathfrak{S}$ be a Cartan subalgebra of $\mathfrak{E} \oplus \mathfrak{L}$. Since $[\mathfrak{E}, \mathfrak{L}]=0$, ad $\mathfrak{\complement}$ normalizes $\mathfrak{S}$ and thus $\mathfrak{S} \subseteq \mathfrak{S}_{\text {. }}$. Hence if $\mathfrak{S}_{1}$ is the projection of $\mathfrak{F}$ on $\mathfrak{Q}$, then $\mathfrak{F}_{1} \subseteq \mathfrak{F}_{2}$. This means that $\mathfrak{H}_{1}=\mathfrak{F} \cap \mathfrak{L}$ and $\mathfrak{S}=\mathfrak{C} \oplus \mathfrak{S}_{1} . \mathfrak{S}_{1}$ is a homomorphic image of $\mathfrak{S}_{\mathfrak{Z}}$ and so is nilpotent. If $x \in \mathfrak{L}$ is such that $\left[\mathfrak{S}_{1}, x\right] \subseteq \mathfrak{S}_{1}$, then $\left[\mathfrak{S}_{2}, x\right]=[\mathfrak{\complement}, x]+\left[\mathfrak{S}_{1}, x\right] \subseteq 0 \oplus \mathfrak{S}_{1}$. Thus $x \in \mathfrak{F}$, i.e. $x \in \mathfrak{F}_{\mathcal{C}} \cap \mathfrak{Q}=\mathfrak{S}_{1}$. This shows that $\mathfrak{S}_{1}$ is a Cartan subalgebra of $\mathfrak{R}$.

Proposition 1. Let $\mathfrak{W}$ be a Cartan subalgebra of $\mathfrak{D}=$ Der $\mathfrak{\Im}$ and let $\mathfrak{A}$ $=\{x \in \mathfrak{I} \mid x \mathfrak{W}=0\}$. Then $\mathfrak{S}=R_{\mathfrak{A}}+\mathfrak{W}$ is the unique Cartan subalgebra of $\mathfrak{Q}$ containing $\mathfrak{i 3}$.

Proof. We first show that $\operatorname{ad}_{\mathfrak{L}} \mathfrak{W}$ is a completely reducible algebra of linear transformations of $\mathfrak{L}$. Indeed, $\mathfrak{D}=\mathfrak{E} \oplus \mathfrak{D}^{\prime}$ and so by the lemma $\mathfrak{W}=\mathbb{E} \oplus \mathfrak{W}_{1}$, where $\mathfrak{W}_{1}=\mathfrak{W} \cap \mathfrak{D}^{\prime}$ is a Cartan subalgebra of $\mathfrak{D}^{\prime}$. Now $\mathbb{E}$ is an abelian Lie algebra of semisimple transformations [8, p. 47]; standard results of the representation theory of semisimple Lie algebras [1, Exposé 17] show that the same is true of $\mathfrak{W}_{1}$. Since $\left[\mathfrak{E}, \mathfrak{W}_{1}\right]=0, \mathfrak{W}$ is also a commutative Lie algebra of semisimple transformations of $\mathfrak{\Im}$ (the sum of commuting semisimple transformations is semisimple). Hence $\mathfrak{W}$ is completely reducible $[8$, p. 100]. Now the adjoint action of $\mathfrak{B}$ in $\operatorname{Hom}_{\Phi}(\Im, \Im)$ is completely reducible [8, pp. 22 and 83]. The restriction of this action to $\mathfrak{L}$ is $\operatorname{ad}_{\mathfrak{L}} \mathfrak{W}$, which is thus completely reducible.

$\mathfrak{W} \subseteq \mathscr{D} \subseteq \mathfrak{R}^{\prime}$ and $\mathfrak{R}^{\prime}$ is semisimple. Since ad $\mathfrak{E} \mathfrak{W}$ is completely reducible and stabilizes $\mathfrak{R}^{\prime}, \operatorname{ad}_{\mathfrak{R}^{\prime}} \mathfrak{W}$ is also completely reducible. But $\mathfrak{W}$ is abelian (since $\mathfrak{W}_{1}$ is); thus we can apply $[8$, p. 105 , exercise 21$]$ to conclude that $\mathfrak{W}$ is contained in some Cartan subalgebra $\mathfrak{H}_{1}$ of $\mathfrak{L}^{\prime}$. Using the lemma again, we conclude that $\mathfrak{W}$ is contained in some Cartan subalgebra of $\mathfrak{L}$, namely $\mathfrak{C} \oplus \mathfrak{S}_{1}$. 
Let $\mathfrak{S}$ be any Cartan subalgebra of $\mathfrak{L}$ containing $\mathfrak{W}$. Then $\mathfrak{F}=\mathfrak{C} \oplus \mathfrak{S}_{1}$, where $\mathfrak{S}_{1}$, a Cartan subalgebra of $\mathfrak{L}^{\prime}$, is abelian. This means that $\mathfrak{S}$ is abelian. Let $R_{x}$ $+D \in \mathfrak{S}$ and $E \in \mathfrak{W}$. Then $0=\left[R_{x}+D, E\right]=R_{x E}+[D, E]$. I.e., $x \mathfrak{W}=0$ and $[D, \mathfrak{W}]$ $=0$; in particular $D$ normalizes $\mathfrak{W}$. Hence $x \in \mathfrak{A}$ and $D \in \mathfrak{W}$. This means that $\mathfrak{H} \subseteq R_{\mathfrak{A}}+\mathfrak{W}$.

$R_{\mathfrak{A}}+\mathfrak{W}$ is a subalgebra of $\mathfrak{L}$. In fact $\left[R_{\mathfrak{U}}, \mathfrak{W}\right]=R_{\mathfrak{U}}=0$; and if $x, y \in \mathfrak{A}$ and $D \in \mathfrak{W}$ then $\left[\left[R_{x}, R_{y}\right], D\right]=\left[\left[R_{x}, D\right], R_{y}\right]+\left[R_{x},\left[R_{y}, D\right]\right]=0+0$ so that $\left[R_{x}, R_{y}\right]$ normalizes $\mathfrak{W}$ and hence is in $\mathfrak{W}$. Thus $\left[R_{\mathfrak{A}}, R_{\mathfrak{U}}\right] \subseteq \mathfrak{W}$.

$R_{\mathfrak{U}}+\mathfrak{W}$ is nilpotent: $\left[R_{\mathfrak{U}}+\mathfrak{W}, \quad R_{\mathfrak{U}}+\mathfrak{W}\right] \subseteq\left[R_{\mathfrak{U}}, R_{\mathfrak{U}}\right]+\left[R_{\mathfrak{U}}, \mathfrak{W}\right]+[\mathfrak{W}, \mathfrak{W}]=\left[R_{\mathfrak{U}}\right.$, $\left.R_{\mathfrak{U}}\right]$ and $\left[\left[R_{\mathfrak{U}}, R_{\mathfrak{A}}\right], R_{\mathfrak{U}}+\mathfrak{W}\right] \subseteq\left[\mathfrak{W}, R_{\mathfrak{A}}+\mathfrak{W}\right]=0$. Since a Cartan subalgebra is a maximal nilpotent subalgebra [8, p. 103, exercise 1], we have $\mathfrak{E}=R_{\mathfrak{A}}+\mathfrak{W}$.

PROPOSITION 2. Let $\mathfrak{\Im}$ be any Jordan algebra with $1, \mathfrak{2}$ the structure Lie algebra of $\mathfrak{\Im}$ and $\mathfrak{R}$ the Koecher-Tits algebra of $\mathfrak{\Im}$. If $\mathfrak{S}$ is a Cartan subalgebra of $\mathfrak{L}$, then $\mathfrak{\mathcal { B }}$ is also a Cartan subalgebra of $\mathfrak{\Re}$.

Proof. $\mathfrak{S}$ is certainly a nilpotent subalgebra of $\mathfrak{R}$. $\left[R_{1}, \mathfrak{S}\right]=0$ since $R_{1}$ is the identity map of $\Im$; so $R_{1}$ normalizes $\mathfrak{S}$-i.e. $R_{1} \in \mathfrak{S}$. Now suppose $a+\bar{b}+L \in \mathfrak{A}$ normalizes $\mathfrak{S}$. Then $a-\bar{b}=a .1-(b .1)^{-}+0=a R_{1}+\left(b\left(-R_{1}\right)\right)^{-}+\left[L, R_{1}\right]=\left[a+\bar{b}+L, R_{1}\right]$ $\in \mathfrak{S} \subseteq \mathfrak{R}$. Hence $a=b=0$, so that $L$ normalizes $\mathfrak{H}$. We conclude that $a+\bar{b}+L$ $=L \in \mathfrak{S}$.

Henceforth $\mathfrak{W}$ will be a fixed, but arbitrary, Cartan subalgebra of $\mathfrak{D}$. We let $\mathfrak{W}_{1}=\mathfrak{W} \cap \mathfrak{D}^{\prime}, \mathfrak{A}=\{x \in \mathfrak{I} \mid x \mathfrak{W}=0\}, \mathfrak{H}=R_{\mathfrak{U}}+\mathfrak{W}, \mathfrak{H}_{1}=\mathfrak{S} \cap \mathfrak{L}^{\prime}$. Propositions 1 and 2 show that $\mathfrak{S}$ is a Cartan subalgebra of $\mathfrak{A}$ and is therefore abelian. Let $\mathfrak{H}^{\prime}=\mathfrak{A} \cap \mathfrak{J}^{\prime}$; then $\mathfrak{S}_{1}=\left(R_{\mathfrak{U}} \oplus \mathfrak{W}\right) \cap\left(R_{\mathfrak{S}^{\prime}} \oplus \mathfrak{D}\right)=R_{\mathfrak{U}^{\prime}} \oplus \mathfrak{W}$.

By ( , ) we will mean the Killing form of $\mathfrak{\Omega}$ and by $\langle$,$\rangle the Killing form of \mathfrak{Q}^{\prime}$. The form ( , ) is nondegenerate on $\mathfrak{K}$, the Cartan subalgebra of $\AA$ constructed above. Associated with (, ) in a natural way is a symmetric bilinear form on $\mathfrak{S}^{*}$, the dual space of $\mathfrak{F}$. The same symbol will be used for this form, which is defined as follows: If $\alpha, \beta \in \mathfrak{S}^{*}$, we choose $H_{\alpha}, H_{\beta} \in \mathfrak{F}$ so that for all $H \in \mathfrak{S},\left(H_{\alpha}, H\right)=\alpha(H)$ and $\left(H_{\beta}, H\right)=\beta(H)$; then $(\alpha, \beta)=\left(H_{\alpha}, H_{\beta}\right)$. Similarly, $\langle$,$\rangle is a nondegenerate$ bilinear form on $\mathfrak{S}_{1}$ and gives rise to a nondegenerate form $\langle$,$\rangle on \mathfrak{H}_{1}^{*}$.

Proposition 3. $\mathfrak{L}^{\prime}=R_{\mathfrak{Y}^{\prime}} \oplus \mathfrak{E} \oplus \mathfrak{D}^{\prime}$, where the three spaces $R_{\mathfrak{Y}^{\prime}}$, $\mathfrak{E}$, and $\mathfrak{D}^{\prime}$ are mutually orthogonal with respect to $\langle$,$\rangle .$

Proof. We first show that $R_{\mathfrak{J}}$ and $\mathfrak{D}$ are orthogonal. If $a \in \mathfrak{I}^{\prime}$ and $D \in \mathfrak{D}$ then $R_{\mathfrak{Y}^{\prime}}$ ad $R_{a}$ ad $D=\left[\left[R_{\mathfrak{Y}^{\prime}}, R_{a}\right], D\right] \subseteq[\mathfrak{D}, D] \subseteq \mathscr{D}$ and $\mathfrak{D}$ ad $R_{a}$ ad $D=\left[\left[\mathscr{D}, R_{a}\right], D\right]$ $\subseteq\left[R_{a} \mathfrak{D}, D\right] \subseteq\left[R_{\mathfrak{J}^{\prime}}, D\right] \subseteq R_{\mathfrak{J}^{\prime}} \subseteq R_{\mathfrak{J}^{\prime}}$. Thus ad $R_{a}$ ad $D$ interchanges the spaces $R_{\mathfrak{J}^{\prime}}$ and $\mathfrak{D}$. It follows that $\left\langle R_{a}, D\right\rangle=\operatorname{tr}$ ad $R_{a}$ ad $D=0$.

Since $\mathfrak{D}^{\prime}$ is spanned by all products $[D, F], D, F \in \mathfrak{D}$, to show that $\left\langle\mathfrak{E}, \mathfrak{D}^{\prime}\right\rangle=0$ we need only to show that $\langle E,[D, F]\rangle=0$ for every $E \in \&, D, F \in \mathcal{D}$. Now $0=\mathfrak{D}$ ad $E$ ad $[D, F]$. Also $E[D, F]=E(D F-F D)=E D F-E F D=E D F-F E D$ $=[E D, F]$. Thus if $a \in \Im^{\prime}, R_{a}$ ad $E$ ad $[D, F]=\left[\left[R_{a}, E\right],[D, F]\right]=R_{a[E D, F]}$. This means that $\langle E,[D, F]\rangle=\operatorname{tr}_{R_{\mathfrak{Y}}}$ ad $E$ ad $[D, F]=\operatorname{tr}_{\mathfrak{I}}[E D, F]=0$. 
COROLLARY. $\mathfrak{S}_{1}=R_{\mathfrak{U}^{\prime}} \oplus \Subset \oplus \mathfrak{W}_{1}$, where $R_{\mathfrak{U}^{\prime}}$, $E$, and $\mathfrak{W}_{1}$ are mutually orthogonal with respect to $\langle$,$\rangle .$

We define the linear transformation $\alpha \mapsto \hat{\alpha}$ from $\mathfrak{S}_{1}^{*}$ to $\mathfrak{S}^{*}$ to be the dual of the natural projection of $\mathfrak{S}_{\mathrm{S}}=\mathfrak{C} \oplus \mathfrak{S}_{1}$ onto $\mathfrak{S}_{1}$. Thus if $\alpha \in \mathfrak{S}_{1}^{*}, C \in \mathfrak{C}$ and $H \in \mathfrak{S}_{1}$ then $\hat{\alpha}(C+H)=\alpha(H)$. Note that $\hat{\alpha}(\mathfrak{C})=0$ and $\hat{\alpha} \mid \mathfrak{S}_{1}=\alpha$. Thus the mapping $\alpha \mapsto \hat{\alpha}$ is injective. The image $\left(\mathfrak{S}_{1}^{*}\right)^{\wedge}$ consists of all $\rho \in \mathfrak{S}^{*}$ such that $\rho(\mathbb{E})=0$. For if $\rho$ has this property then $\left(\rho \mid \mathfrak{S}_{1}\right)^{\wedge}=\rho$.

In a similar way we define the linear transformation $\omega \mapsto \bar{\omega}$ from $\mathfrak{W}_{1}^{*}$ to $\mathfrak{S}_{1}^{*}$ to be the dual of the natural projection of $\mathfrak{S}_{1}=R_{\mathfrak{U}^{\prime}} \oplus \mathfrak{E} \oplus \mathfrak{W}_{1}$ onto $\mathfrak{W}_{1}$. Thus if $\omega \in \mathfrak{W}_{1}^{*}, a \in \mathfrak{A}^{\prime}, E \in \mathfrak{E}$ and $H \in \mathfrak{W}_{1}$ then $\bar{\omega}\left(R_{a}+E+H\right)=\omega(H)$. The mapping $\omega$ $\mapsto \bar{\omega}$ is injective and the image $\left(\mathfrak{W}_{1}^{*}\right)^{-}$consists of all $\alpha \in \mathfrak{S}_{1}^{*}$ such that $\alpha\left(R_{\mathfrak{U}}\right)=\alpha(\mathbb{E})$ $=0$. We let $\mathfrak{F}^{*}=\left\{\alpha \in \mathfrak{S}_{1}^{*} \mid \alpha\left(R_{\mathfrak{U}^{\prime}}\right)=\alpha\left(\mathfrak{W}_{1}\right)=0\right\}$ and $\mathfrak{A}^{*}=\left\{\alpha \in \mathfrak{S}_{1}^{*} \mid \alpha(\mathfrak{F})=\alpha\left(\mathfrak{W}_{1}\right)=0\right\}$. With these definitions $\mathfrak{S}_{1}^{*}=\mathfrak{H}^{*} \oplus \mathfrak{F}^{*} \oplus\left(\mathfrak{W}_{1}^{*}\right)^{-}$, as is easily verified (the inconsistency in notation here is deliberate: although $\mathfrak{A}^{*}$ and $\mathfrak{F}^{*}$ can be naturally identified with the dual spaces of $R_{\mathfrak{A}}$ and $\mathfrak{E}$, respectively, we shall not need to use this fact; we will, however, make explicit use of the identification of $\left(\mathfrak{W}_{1}^{*}\right)^{-}$with the dual space of $\left.\mathfrak{W}_{1}\right)$. The Corollary above implies that the three spaces $\mathfrak{A}^{*}$, $\mathfrak{F}^{*}$, and $\left(\mathfrak{W}_{1}^{*}\right)^{-}$are mutually orthogonal with respect to $\langle$,$\rangle . Indeed, let \alpha \in \mathfrak{A}^{*}, \beta \in \mathfrak{F}^{*}$, $\gamma \in\left(\mathfrak{W}_{1}^{*}\right)^{-}$and choose $H_{\alpha}, H_{\beta}, H_{\gamma}$ in $\mathfrak{S}_{1}$ so that for all $H \in \mathfrak{S}_{1},\left\langle H_{\alpha}, H\right\rangle=\alpha(H)$, $\left\langle H_{\beta}, H\right\rangle=\beta(H)$, and $\left\langle H_{\gamma}, H\right\rangle=\gamma(H)$. Since $\left\langle H_{\alpha}, \mathfrak{E}+\mathfrak{W}_{1}\right\rangle=0$ and since the spaces ⿷匚 and $\mathfrak{W}_{1}$ are nonisotropic with respect to $\langle$,$\rangle , it follows that H_{\alpha} \in R_{\mathfrak{A}^{\prime}}$. Similarly $H_{\beta} \in \mathbb{E}$ and $H_{\gamma} \in \mathfrak{W B}_{1}$. But then $\langle\alpha, \beta\rangle=\left\langle H_{\alpha}, H_{\beta}\right\rangle=0,\langle\alpha, \gamma\rangle=\left\langle H_{\alpha}, H_{\gamma}\right\rangle=0$ and $\langle\beta, \gamma\rangle=\left\langle H_{\beta}, H_{\gamma}\right\rangle=0$. For brevity we let $\mathfrak{W}^{*}=\mathfrak{F}^{*} \oplus\left(\mathfrak{W}_{1}^{*}\right)^{-}$in the sequel.

The automorphism $\varepsilon$ of $\mathfrak{\Re}$ has period two and stabilizes $\mathfrak{S}$ and $\mathfrak{S}_{1}$. We denote by $\varepsilon^{*}$ both the dual of $\varepsilon \mid \mathfrak{S}$ (acting in $\mathfrak{S}^{*}$ ) and the dual of $\varepsilon \mid \mathfrak{S}_{1}$ (acting in $\mathfrak{H}_{1}^{*}$ ). These two transformations agree in the following sense: if $\alpha \in \mathfrak{S}_{1}^{*}$ then $\left(\alpha \varepsilon^{*}\right)^{\wedge}=\hat{\alpha} \varepsilon^{*}$. Indeed, if $R_{a}+D \in \mathfrak{H}_{1}$ then $\left(\hat{\alpha} \varepsilon^{*}\right)\left(R_{a}+D\right)=\hat{\alpha}\left(\left(R_{a}+D\right) \varepsilon\right)=\hat{\alpha}\left(-R_{a}+D\right)=\alpha\left(-R_{a}+D\right)=$ $\alpha \varepsilon^{*}\left(R_{a}+D\right)=\left(\alpha \varepsilon^{*}\right)^{\wedge}\left(R_{a}+D\right)$. Now if $R_{a}+D$ is in the center of $\mathfrak{L}$ then $D=0$, since for all $x \in \Im, 0=\left[R_{x}, R_{a}+D\right]=R_{x D}+\left[R_{x}, R_{a}\right]$, i.e. $x D=0$. This means that if $C \in \mathfrak{C}$ then $C \varepsilon=-C$ so that $\hat{\alpha} \varepsilon^{*}(C)=\hat{\alpha}(-C)=0=\left(\alpha \varepsilon^{*}\right)^{\wedge}(C)$.

If $\alpha \in \mathfrak{Y}^{*}$ and $\omega \in \mathfrak{W}^{*}$ then $\alpha \varepsilon^{*}=-\alpha$ and $\omega \varepsilon^{*}=\omega$. To see this, one merely observes that for all $R_{a}+D \in \mathfrak{H}_{1}$ one has $\left(\alpha \varepsilon^{*}\right)\left(R_{a}+D\right)=\alpha\left(-R_{a}+D\right)=\alpha\left(-R_{a}\right)=$ $-\alpha\left(R_{a}\right)=(-\alpha)\left(R_{a}+D\right)$ and $\left(\omega \varepsilon^{*}\right)\left(R_{a}+D\right)=\omega\left(-R_{a}+D\right)=\omega(D)=\omega\left(R_{a}+D\right)$. This means that $\mathfrak{U}^{*}=\left\{\alpha \in \mathfrak{S}_{1}^{*} \mid \alpha \varepsilon^{*}=-\alpha\right\}$ and $\mathfrak{Y B}^{*}=\left\{\alpha \in \mathfrak{S}_{1}^{*} \mid \alpha \varepsilon^{*}=\alpha\right\}$. As a consequence, we see that for $\alpha \in \mathfrak{S}_{1}^{*}$, the projection of $\alpha$ on $\mathfrak{W}^{*}$ is $\alpha_{+}=\frac{1}{2}\left(\alpha+\alpha \varepsilon^{*}\right)$, whereas the projection of $\alpha$ on $\mathfrak{A}^{*}$ is $\alpha_{-}=\frac{1}{2}\left(\alpha-\alpha \varepsilon^{*}\right)$. In fact, $\varepsilon^{*}$ fixes $\frac{1}{2}\left(\alpha+\alpha \varepsilon^{*}\right)$ and sends $\frac{1}{2}\left(\alpha-\alpha \varepsilon^{*}\right)$ to $-\frac{1}{2}\left(\alpha-\alpha \varepsilon^{*}\right)$, so that $\frac{1}{2}\left(\alpha+\alpha \varepsilon^{*}\right) \in \mathfrak{W}^{*}$ and $\frac{1}{2}\left(\alpha-\alpha \varepsilon^{*}\right) \in \mathfrak{H}^{*}$; and certainly $\alpha=\frac{1}{2}\left(\alpha+\alpha \varepsilon^{*}\right)+\frac{1}{2}\left(\alpha-\alpha \varepsilon^{*}\right)$.

We now turn our attention to the relationship between the groups $G$ and $\Gamma$ and these algebras. If $\eta \in \Gamma$, then the linear transformation $\tilde{\eta}: a+\vec{b}+L \rightarrow a+\left(b \eta^{\#-1}\right)^{-}$ $+\eta^{-1} L \eta$ of $\AA$ into itself is an automorphism of $\Re$. If $\eta, \zeta \in \Gamma$ then $\tilde{\eta} \tilde{\zeta}=(\eta \zeta)^{\sim}$ (see, 
for example, p. 33 of [12]). The mapping $\eta \mapsto \tilde{\eta}$ of $\Gamma$ into Aut $\Re$ is clearly a rational homomorphism, since $\eta^{\#-1}=\eta U_{1 \eta}^{-1}$. Since $\tilde{\eta} \mid \Im=\eta$, the mapping $\eta \mapsto \tilde{\eta}$ is in fact a birational isomorphism between $\Gamma$ and a subgroup $\tilde{\Gamma}$ of Aut $\Re$. The fact that the mapping is birational implies that it makes the algebraic components of $\Gamma$ and $\tilde{\Gamma}$ correspond. In particular, $(\tilde{\Gamma})_{0}=\left(\Gamma_{0}\right)^{\sim}$. The same considerations apply to the restriction of this automorphism to $G$; thus the components of $G$ and $\tilde{G}$ correspond and $(\widetilde{G})_{0}=\left(G_{0}\right)^{\sim}$. We can characterize the group $\tilde{\Gamma}$ as follows.

PROPOSITION 4. The following are equivalent:

(i) $\chi \in \tilde{\Gamma}$;

(ii) $\chi$ is an automorphism of $\Re$ fixing $R_{1}$;

(iii) $\chi$ is an automorphism of $\mathfrak{\Re}$ stabilizing $\mathfrak{\Im}$ and $\mathfrak{\Im}$.

Proof. (i) implies (ii): If $\eta \in \Gamma$ is such that $\tilde{\eta}=\chi$, then $R_{1} \chi=\eta^{-1} R_{1} \eta=\eta^{-1} I \eta=I$ $=R_{1}$.

(ii) implies (iii): Let $a, b \in \mathfrak{s}$. Then $a \chi=\left[a, R_{1}\right] \chi=\left[a \chi, R_{1} \chi\right]=\left[a \chi, R_{1}\right]$ and $\bar{b} \chi$ $=\left((-b)\left(-R_{1}\right)\right)^{-} \chi=\left[-\bar{b}, R_{1}\right] \chi=\left[-\bar{b} \chi, R_{1}\right]=-\left[\bar{b} \chi, R_{1}\right]$. Now in general, if $c, d \in \Im$ and $L \in \mathfrak{Q}$ then $\left[c+\bar{d}+L, R_{1}\right]=c-\bar{d}+0$ so that $c+\bar{d}+L=\left[c+\bar{d}+L, R_{1}\right]$ means $d=L=0$ and $c+\bar{d}+L=-\left[c+\bar{d}+L, R_{1}\right]$ means $c=L=0$. Hence $a \chi \in \Im$ and $\bar{b} \chi \in \varsubsetneqq$.

(iii) implies (i): For any $a \in \mathfrak{\Im}, R_{a \chi}=\left(R_{a .1}-\left(R_{a}, R_{1}\right]\right) \chi=\frac{1}{2}[a, \overline{1}] \chi=\frac{1}{2}[a \chi, \overline{1} \chi] \in \mathfrak{R}$, since $a_{\chi} \in \mathfrak{\Im}$ and $\overline{1} \chi \in \bar{\Im}$. Since $R_{\mathfrak{I}}$ generates $\mathfrak{Q}$ we have $\mathfrak{Q} \chi \subseteq \mathfrak{Q}$.

Let $\eta=\chi \mid \Im$. Then if $L \in \mathfrak{R}, a \in \Im$ it follows that $a \eta^{-1} L \eta=\left[a \chi^{-1}, L\right] \chi=\left[a \chi^{-1} \chi, L \chi\right]$ $=\left[a, L_{\chi}\right]=a\left(L_{\chi}\right)$. Thus $\eta^{-1} L_{\eta}=L_{\chi}$.

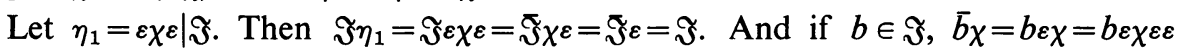
$=b \eta_{1} \varepsilon=\left(b \eta_{1}\right)^{-}$. Now $\eta^{-1}\left(R_{a, b}-\left[R_{a}, R_{b}\right]\right) \eta=\frac{1}{2}[a, \bar{b}] \chi=\frac{1}{2}\left[a \eta,\left(b \eta_{1}\right)^{-}\right]=R_{a \eta, b \eta_{1}}-$ $\left[R_{a \eta}, R_{b \eta_{1}}\right]$. We let both sides of this equation operate on $a \eta$, and use the fact $[10, \mathrm{p}$. 325] that for $a, b, c \in \Im$ one has $c\left(R_{a . b}-\left[R_{a}, R_{b}\right]\right)=\{a b c\} \equiv b U_{a, c}$ (here $U_{a, c}$ means $\left.\frac{1}{2}\left(U_{a+c}-U_{a}-U_{c}\right)\right)$. We conclude that $\{a, b, a\} \eta=\left\{a \eta, b \eta_{1}, a \eta\right\}$, i.e. that $b U_{a} \eta$ $=b \eta_{1} U_{a \eta}$. Therefore $U_{a} \eta=\eta_{1} U_{a \eta}$ and it follows that $\eta \in \Gamma$ and $\eta_{1}=\eta^{\#-1}$. This proves that $\chi=\tilde{\eta}: a+\bar{b}+L \rightarrow a \eta+\left(b \eta^{\#-1}\right)^{-}+\eta^{-1} L \eta$.

COROLlaRY. If $\chi$ is an automorphism of $\mathfrak{\AA}$, then $\chi \in \tilde{G}$ if and only if $R_{1} \chi=R_{1}$ and $1 \chi=1$.

We note that $\tilde{G}_{0} \subseteq \tilde{\Gamma}_{0} \subseteq$ Aut $_{0} \mathfrak{R}$, the component of the identity of Aut $\mathfrak{R}$. This follows from the fact that $\widetilde{G}_{0}$ and $\tilde{\Gamma}_{0}$ are irreducible and contain 1 , together with the following general observation: If $A$ is a linear group and $S$ an irreducible subset of $A$, then $S$ is entirely contained in some algebraic component of $A$. This observation also shows that if $G_{i}$ is any component of $G$, then $G_{i}$ lies entirely in some component $\Gamma_{j}$ of $\Gamma$, and $\tilde{\Gamma}_{j}$ lies entirely in some component of Aut $\Re$.

If $\eta \in G$, then $\eta^{-1} R_{a} \eta=R_{a \eta}$. This implies that $\tilde{\eta}$ stabilizes $R_{\mathfrak{I}}$ and also that $\tilde{\eta}$ stabilizes $\mathfrak{D}=\left[R_{\mathfrak{J}}, R_{\mathfrak{I}}\right]$. Hence $\tilde{\eta}$ must also stabilize $\&$ and $\mathfrak{D}^{\prime}$. We let $\check{\eta}=\tilde{\eta} \mid \mathfrak{D}^{\prime}$. Then $\eta \mapsto \check{\eta}$ is a rational homomorphism of $G$ into Aut $\mathfrak{D}^{\prime}$. 
Proposition 5. If $\eta \in G_{0}$ then $\tilde{\eta}$ fixes $\mathbb{E}$ pointwise. $\breve{G}_{0}$ is the component of the identity of Aut $\mathfrak{D}^{\prime}$.

Proof. Since the field has characteristic zero, $D$ is the Lie algebra of $G_{0}$ and the subalgebras of $\operatorname{Hom}_{\Phi}(\mathfrak{\Im}, \mathfrak{\Im})$ generated by $\mathfrak{D}$ and by $G_{0}$ are the same [2, Vol. II, Théorème 16, p. 179 and Corollaire 2, p. 157]. Certainly \& is in the center of this subalgebra. Hence every element of $G_{0}$ commutes with every element of $₹$. This proves the first assertion.

Since the field is algebraically closed, $\breve{G}_{0}$ is an algebraic group acting on $\mathfrak{D}^{\prime}[2$, Vol. II, Corollaire 1, p. 122]. Previous considerations have established that $\breve{G}_{0}$ $\subseteq$ Aut $_{0} \mathfrak{D}^{\prime}$. So to show equality, we need only show that $\operatorname{dim}_{\Phi} \breve{G}_{0} \geqq \operatorname{dim}_{\Phi}$ Aut $_{0} \mathfrak{D}^{\prime}$. This can be accomplished by showing that the Lie algebra of $\breve{G}_{0}$ contains the Lie algebra of $\operatorname{Aut}_{0} \mathfrak{D}^{\prime}$, i.e. contains Der $\mathfrak{D}^{\prime}=\operatorname{ad}_{\mathfrak{D}^{\prime}} \mathfrak{D}^{\prime}$ (since $\mathfrak{D}^{\prime}$ is semisimple). We do this as follows:

We consider the adjoint representation of $G_{0}$, under which each $\eta \in G_{0}$ is mapped to the linear transformation ad $\eta$ of $\mathscr{D}$ given by $D \mapsto \eta^{-1} D \eta$ for all $D \in \mathscr{D}$. Now ad $G_{0}$ is an algebraic group whose Lie algebra contains $\operatorname{ad}_{\mathfrak{D}} \mathfrak{D}[2$, Vol. II, Théorème 6, p. 137; Définition 1, p. 138; Proposition 7, p. 142]. $G_{0}$ is the restriction of ad $G_{0}$ to $\mathfrak{D}^{\prime}$. Let $f$ be the mapping from $\operatorname{Hom}_{\Phi}(\mathfrak{D}, \mathfrak{D})$ to $\operatorname{Hom}_{\Phi}\left(\mathfrak{D}^{\prime}, \mathfrak{D}^{\prime}\right)$ which sends $\zeta \in \operatorname{Hom}(\mathfrak{D}, \mathfrak{D})$ to the linear transformation $\zeta f$ such that for $D \in \mathfrak{D}^{\prime}, D(\zeta f)$ is the projection of $D \zeta$ onto $\mathfrak{D}^{\prime}$ (with respect to the decomposition $\mathfrak{D}=\mathfrak{E} \oplus \mathfrak{D}^{\prime}$ ). Then $f$ gives the rational representation of ad $G_{0}$ such that $\left(\operatorname{ad} G_{0}\right) f=\breve{G}_{0}$. So the Lie algebra of $\check{G}_{0}$ contains the image of that of ad $G_{0}$ under the differential of $f$. Since $f$ is linear, it is its own differential. In particular, the Lie algebra of $\breve{G}_{0}$ contains $(\operatorname{ad} \mathfrak{D} \mathfrak{D}) f=\operatorname{ad}_{\mathfrak{D}} \mathfrak{D}^{\prime}$.

2. Roots. We will be interested in several root systems: the root systems associated with the semisimple Lie algebras $\mathfrak{R}, \mathfrak{L}^{\prime}$, and $\mathfrak{D}^{\prime}$, and one other abstract root system which will be defined presently. The definition of root system we shall use is that of Serre [13, p. V-3]. Thus by a root system we mean a finite subset $\Sigma$ of a rational vector space $\mathfrak{B}$ equipped with a positive definite symmetric bilinear form $($,$) , such that the following conditions are satisfied:$

(i) $\Sigma$ spans $\mathfrak{B}$ and does not contain 0 ;

(ii) for every $\alpha \in \Sigma$, the symmetry $w_{\alpha}$ of $\mathfrak{B}$ in the direction of $\alpha$ stabilizes $\Sigma$ (here $w_{\alpha}: x \rightarrow x-(2(x, \alpha) /(\alpha, \alpha)) \alpha$ for all $\left.x \in \mathfrak{B}\right)$;

(iii) for every $\alpha, \beta \in \Sigma, 2(\alpha, \beta) /(\alpha, \alpha)$ is an integer.

The elements of $\Sigma$ are called roots; $\Sigma$ is reduced if for each $\alpha \in \Sigma$ and $\lambda \in \Phi$, $\lambda \alpha \in \Sigma$ implies $\lambda=+1$ or -1 . It is proven in [13, Chapitre VI] that the reduced root systems are exactly those corresponding to semisimple Lie algebras.

We first consider the relationship between the roots of $\mathfrak{L}^{\prime}$ (which are elements of $\mathfrak{S}_{1}^{*}$ ) and those of $\mathfrak{\Re}$ (which belong to $\mathfrak{S}^{*}$ ). Note that $R_{1} \in \mathfrak{C} \subseteq \mathfrak{H}$.

Proposition 6. (a) If $\rho$ is a root of $\AA$ then the root space $\mathfrak{\Omega}_{\rho}$ of $\rho$ is contained

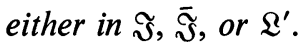


(b) If $\rho$ is a root of $\mathfrak{R}$, then $\rho\left(R_{1}\right)=+1,-1$, or 0 according as $\mathfrak{R}_{\rho}$ is contained in $\mathfrak{\Im}, \bar{\Im}$, or $\mathfrak{L}^{\prime}$ respectively.

(c) If $\alpha$ is a root of $\mathfrak{L}^{\prime}$ then $\hat{\alpha}$ is a root of $\mathfrak{R}$ satisfying $\hat{\alpha}\left(R_{1}\right)=0$. If $\rho$ is a root of $\mathfrak{\Re}$ satisfying $\rho\left(R_{1}\right)=0$, then $\alpha=\rho \mid \mathfrak{F}_{1}$ is the unique root of $\mathfrak{R}^{\prime}$ such that $\hat{\alpha}=\rho$.

Proof. (a) Let $0 \neq K \in \mathfrak{\Re}_{\rho}$, say $K=a+\bar{b}+L$, where $a, b \in \mathfrak{\Im}, L \in \mathfrak{L}$. Then for any $H \in \mathfrak{H}, \quad \rho(H) a+\rho(H) \bar{b}+\rho(H) L=[K, H]=[a, H]+[\bar{b}, H]+[L, H] . \quad$ Since $\quad \operatorname{ad}_{\mathfrak{A}} \mathfrak{S}$ stabilizes $\mathfrak{\jmath}, \bar{\Im}$, and $\mathfrak{Q}$ we have $\rho(H) a=[a, H], \rho(H) \bar{b}=[\bar{b}, H]$, and $\rho(H) L=[L, H]$. But $\mathfrak{A}_{\rho}$ is 1-dimensional [8, p. 111], so at most one of $a, \bar{b}, L$ can be nonzero. Thus $\mathfrak{\Re}_{\rho}=\Phi a, \Phi \bar{b}$, or $\Phi L$. If $L \neq 0$, choose $H$ so that $\rho(H) \neq 0$. Then $\left[\rho(H)^{-1} L, H\right]=L$, i.e. $L \in \mathfrak{Q}^{\prime}$.

(b) Let $0 \neq K \in \mathfrak{\Re}_{\rho}$. Then if $K=a \in \Im,\left[a, R_{1}\right]=a$. If $K=\bar{b} \in \bar{\Im}$, then $\left[\bar{b}, R_{1}\right]$ $=\left(b\left(-R_{1}\right)\right)^{-}=-\bar{b}$. If $K=L \in \mathbb{L}^{\prime}$, then $\left[L, R_{1}\right]=0$.

(c) Let $L \in \mathfrak{R}_{\alpha}^{\prime}$, the root space of $\alpha$. Then $L$ is a root vector for $\hat{\alpha}$ since $[L, H]$ $=\alpha(H) L$ whenever $H \in \mathfrak{S}_{1}$, and $[L, C]=0=0 \cdot L$ whenever $C \in \mathfrak{C}$. Certainly $\hat{\alpha}\left(R_{1}\right)$ $=0$, since $R_{1} \in \mathfrak{C}$. Conversely, if $\rho\left(R_{1}\right)=0$ then $\mathfrak{R} \subseteq \mathfrak{L}^{\prime}$ by (b). Let $0 \neq L \in \mathfrak{\Re}_{\rho}$. Then for any $H \in \mathfrak{S}_{1},[L, H]=\rho(H) L=\left(\rho \mid \mathfrak{S}_{1}\right)(H) L$. Thus $L$ is a root vector for $\alpha=\rho \mid \mathfrak{S}_{1}$. Moreover, for any $C \in \mathfrak{E}, 0=[L, C]=\rho(C) L$, i.e. $\rho(\mathfrak{E})=0$. This means that $\rho=\hat{\alpha}$.

Proposition 7. Let $\alpha_{1}, \ldots, \alpha_{l}$ be a simple system of roots of $\mathfrak{L}^{\prime}$. Then $\hat{\alpha}_{1}, \ldots, \hat{\alpha}_{l}$ can be embedded in a simple system of roots of $\AA$.

Proof. Since $\alpha \mapsto \hat{\alpha}$ is injective, $\hat{\alpha}_{1}, \ldots, \hat{\alpha}_{l}$ are linearly independent in $\mathfrak{S}^{*}$. Choose roots $\rho_{1}, \ldots, \rho_{r}$ of $\Re$ so that $\rho_{1}, \ldots, \rho_{r}, \hat{\alpha}_{1}, \ldots, \hat{\alpha}_{l}$ is a basis for $\mathfrak{S}^{*}$ and hence also for $\mathfrak{H}_{0}^{*}$, the rational vector space spanned by the roots of $\mathfrak{R}$. Let $\leqq$ be the lexicographic ordering on $\mathfrak{S}_{0}^{*}$ associated with this basis of roots (in this order) $\left[8\right.$, p. 119]. Then with this ordering all $\hat{\alpha}_{i}$ are simple. To prove this we suppose that for some $k, \hat{\alpha}_{k}=\rho+\sigma$, where $\rho>0, \sigma>0$ are roots of $\Re$. Now $\rho$ and $\sigma$ must both be linear combinations of $\hat{\alpha}_{1}, \ldots, \hat{\alpha}_{l}$. For otherwise there would exist a $j$ such that $\rho=\lambda_{j} \rho_{j}+\cdots+\lambda_{r} \rho_{r}+\sum_{i} \mu_{i} \hat{\alpha}_{i}$, where $\lambda_{i}, \mu_{i}$ are rational numbers and $\lambda_{j}>0$. But this would mean that $\sigma=\hat{\alpha}_{k}-\rho=-\lambda_{j} \rho_{j}+\cdots<0$, a contradiction.

So suppose $\rho=\sum \lambda_{i} \hat{\alpha}_{i}=\left(\sum \lambda_{i} \alpha_{i}\right)^{\wedge}$. This means that $\rho\left(R_{1}\right)=0$, i.e. (by Proposition 6(c)) that $\sum \lambda_{i} \alpha_{i}=\rho \mid \mathfrak{S}_{1}$ is a root of $\mathfrak{Q}^{\prime}$. Similarly $\sigma=\sum \mu_{i} \hat{\alpha}_{i}$ and $\sum \mu_{i} \alpha_{i}$ is a root of $\mathfrak{R}^{\prime}$. At least one $\lambda_{i}$ and one $\mu_{i}$ are positive since $\rho, \sigma>0$. So $\rho \mid \mathscr{S}_{1}$ and $\sigma \mid \mathscr{F}_{1}$ are positive roots of $\mathfrak{L}^{\prime}$ with respect to the simple system $\alpha_{1}, \ldots, \alpha_{l}$. But $\alpha_{k}=\rho\left|\mathfrak{S}_{1}+\sigma\right| \mathfrak{S}_{1}$, a contradition to the simplicity of $\alpha_{k}$.

Proposition 8. Let $\alpha_{1}, \ldots, \alpha_{l}$ be a simple system of roots of $\mathfrak{L}^{\prime}$. Let $\rho_{1}, \ldots, \rho_{r}$, $\hat{\alpha}_{1}, \ldots, \hat{\alpha}_{l}$ be a simple system of roots of $\Re$. Then if $\rho$ is any root of $\Re$, say

$$
\rho=\sum_{i=1}^{r} n_{i} \rho_{i}+\sum_{i=1}^{l} m_{i} \hat{\alpha}_{i},
$$

it follows that at most one $n_{i}$ is nonzero, and that a nonzero $n_{i}$ is either +1 or -1 .

Proof. Note that $\rho_{i}\left(R_{1}\right)= \pm 1$ for each $i$. Otherwise by Proposition 6(c) we would have $\rho_{i}=\hat{\alpha}$ for some $\alpha \in \mathfrak{S}_{1}^{*}$, i.e. $\rho_{i}$ would be a linear combination of the $\hat{\alpha}_{i}$ 's. By 
replacing $\rho$ by $-\rho$ if necessary, we can assume all $n_{i}, m_{i} \geqq 0$. We apply [8, XVII, p. 123] to this representation of $\rho$. Thus there is a sequence of roots, the last of which is $\rho$, such that each root in the sequence is obtained by adding some simple root to the preceding. If the proposition is false we consider the first root in this sequence of partial sums which contains exactly two of the roots $\rho_{i}$. Thus there exist $\sigma=\sum q_{i} \hat{\alpha}_{i}\left(q_{i} \geqq 0\right)$ and roots $\rho_{i}, \rho_{j}$ so that $\rho_{i}+\sigma, \rho_{i}+\rho_{j}+\sigma$ are roots of $\Re$. Note that $\sigma$ need not be a root.

Case 1. $\rho_{i}\left(R_{1}\right)=\rho_{j}\left(R_{1}\right)=1$. Then $\left(\rho_{i}+\rho_{j}+\sigma\right)\left(R_{1}\right)=2$, in contradiction to Proposition 6(b).

Case 2. $\rho_{i}\left(R_{1}\right)=\rho_{j}\left(R_{1}\right)=-1$. Then $\left(\rho_{i}+\rho_{j}+\sigma\right)\left(R_{1}\right)=-2$, again a contradiction.

Case 3. $\rho_{i}\left(R_{1}\right)=1, \rho_{j}\left(R_{1}\right)=-1$. Then $\left(\rho_{i}+\rho_{j}+\sigma\right)\left(R_{1}\right)=0$. But this would mean that $\rho_{i}+\rho_{j}+\sigma=\hat{\alpha}$ for some root $\alpha$ of $\mathfrak{L}^{\prime}$ by Proposition 6(c). I.e., $\rho_{i}+\rho_{j}$ is a linear combination of $\hat{\alpha}_{1}, \ldots, \hat{\alpha}_{l}$, a contradiction.

Case 4. $\rho_{i}\left(R_{1}\right)=-1, \rho_{j}\left(R_{1}\right)=1$. Then again $\left(\rho_{i}+\rho_{j}+\sigma\right)\left(R_{1}\right)=0$, a contradiction.

Proposition 9. Let $\alpha_{1}, \ldots, \alpha_{l}$ be a simple system of roots of $\mathfrak{L}^{\prime}$. Then there is a unique set $\left\{\rho_{1}, \ldots, \rho_{r}\right\}$ of roots of $\Re$ such that $\rho_{1}\left(R_{1}\right)=\cdots=\rho_{r}\left(R_{1}\right)=1$ and $\left\{\rho_{1}, \ldots\right.$, $\left.\rho_{r}, \hat{\alpha}_{1}, \ldots, \hat{\alpha}_{l}\right\}$ is a simple system of roots of $\Re$.

Proof. By Proposition 7, we can choose roots $\rho_{1}^{\prime}, \ldots, \rho_{r}^{\prime}$ so that $\rho_{1}^{\prime}, \ldots, \rho_{r}^{\prime}$, $\hat{\alpha}_{1}, \ldots, \hat{\alpha}_{l}$ is a simple system. In any event, $\rho_{i}^{\prime}\left(R_{1}\right)= \pm 1$ for $i=1, \ldots, r$. Thus $\rho_{1}^{\prime}\left(R_{1}\right) \rho_{1}^{\prime}, \ldots, \rho_{r}^{\prime}\left(R_{1}\right) \rho_{r}^{\prime}, \hat{\alpha}_{1}, \ldots, \hat{\alpha}_{l}$ is a basis for $\mathfrak{S}_{0}^{*}$ consisting of roots. Let $\leqq$ be the lexicographic ordering on $\mathfrak{S}_{0}^{*}$ associated with this basis of roots (in this order). As in the proof of Proposition 7, $\hat{\alpha}_{1}, \ldots, \hat{\alpha}_{l}$ are simple roots with respect to this ordering. We show that if $\rho$ is any other simple root, then $\rho\left(R_{1}\right)=1$. Now by Proposition $8, \rho=\varepsilon \rho_{j}^{\prime}+\sum_{i} m_{i} \hat{\alpha}_{i}$ for some $j$ and $\varepsilon= \pm 1$, i.e. $\rho=\varepsilon \rho_{j}^{\prime}\left(R_{1}\right) \rho_{j}^{\prime}\left(R_{1}\right) \rho_{j}^{\prime}+\sum_{i} m_{i} \hat{\alpha}_{i}$. Now since $\rho>0$ in this ordering we must have $\varepsilon \rho_{j}^{\prime}\left(R_{1}\right)=1$, i.e. $\varepsilon=\rho_{j}^{\prime}\left(R_{1}\right)$. So $\rho\left(R_{1}\right)=\varepsilon \rho_{j}^{\prime}\left(R_{1}\right)+0=\rho_{j}^{\prime}\left(R_{1}\right) \rho_{j}^{\prime}\left(R_{1}\right)=1$.

Let $\left\{\sigma_{1}, \ldots, \sigma_{r}, \hat{\alpha}_{1}, \ldots, \hat{\alpha}_{l}\right\}$ be a second simple system of roots such that $\sigma_{i}\left(R_{1}\right)$ $=1, i=1, \ldots, r$. Then by Proposition 8 , for each $i, \sigma_{i}=\varepsilon_{i} \rho_{i \pi}+\sum_{j} m_{i j} \hat{\alpha}_{j}$, where $i \pi$ is $1,2, \ldots$, or $r$ and $\varepsilon_{i}= \pm 1$. Since $\left\{\sigma_{1}, \ldots, \sigma_{r}, \hat{\alpha}_{1}, \ldots, \hat{\alpha}_{l}\right\}$ is linearly independent, all the $i \pi$ are distinct-i.e. $\pi$ is a permutation of $\{1, \ldots, r\}$. Now $\varepsilon_{i}=\varepsilon_{i} \rho_{i \pi}\left(R_{1}\right)$ $=\sigma_{i}\left(R_{1}\right)=1$ for all $i$. Thus also all $m_{i j}$ are nonnegative integers. Furthermore, $\rho_{i \pi}=\sigma_{i}-\sum_{j} m_{i j} \hat{\alpha}_{j}$. And since $\left\{\sigma_{1}, \ldots, \sigma_{r}, \hat{\alpha}_{1}, \ldots, \hat{\alpha}_{l}\right\}$ is a simple system, all $m_{i j}$ are nonpositive integers. Hence all $m_{i j}$ are zero and $\sigma_{i}=\rho_{i \pi}$.

Corollary. Let $\rho_{1}, \ldots, \rho_{r}, \alpha_{1}, \ldots, \alpha_{l}$ be as in Proposition 9. Let $\eta$ be a linear transformation of $\mathfrak{S}^{*}$ (or $\mathfrak{S}_{0}^{*}$ ) which permutes the roots of $\AA$, permutes the roots $\rho$ of $\mathfrak{\Re}$ such that $\rho\left(R_{1}\right)=1$, and permutes $\hat{\alpha}_{1}, \ldots, \hat{\alpha}_{l}$. Then $\eta$ also permutes $\rho_{1}, \ldots, \rho_{r}$.

Proof. $\left\{\rho_{1}, \ldots, \rho_{r}, \hat{\alpha}_{1}, \ldots, \hat{\alpha}_{l}\right\} \eta$ is also a simple system of roots of $\mathfrak{R}$. It is equal to $\left\{\rho_{1} \eta, \ldots, \rho_{r} \eta, \hat{\alpha}_{1}, \ldots, \hat{\alpha}_{l}\right\}$, where $\left(\rho_{i} \eta\right)\left(R_{1}\right)=1$ for all $i$. Thus by the uniqueness assertion of Propostion 9, $\left\{\rho_{1} \eta, \ldots, \rho_{r} \eta\right\}=\left\{\rho_{1}, \ldots, \rho_{r}\right\}$.

Proposition 10. Let $\alpha, \beta$ be roots of $\mathfrak{L}^{\prime}$. Then $2\langle\alpha, \beta\rangle /\langle\alpha, \alpha\rangle=2(\hat{\alpha}, \hat{\beta}) /(\hat{\alpha}, \hat{\alpha})$. 
Proof. Let $\hat{\beta}-r \hat{\alpha}, \ldots, \hat{\beta}+q \hat{\alpha}$ be the $\hat{\alpha}$-string of roots through $\hat{\beta}$. Now if $m \alpha+n \beta$ is a root, so is $m \hat{\alpha}+n \hat{\beta}$. Conversely, if $m \hat{\alpha}+n \hat{\beta}=(m \alpha+n \beta)^{\wedge}$ is a root then since it annihilates $R_{1}, m \alpha+n \beta=(m \alpha+n \beta)^{\wedge} \mid \mathfrak{S}_{1}$ is a root of $\mathfrak{Q}^{\prime}$ by Proposition 6(c). So the $\alpha$-string of roots through $\beta$ is $\beta-r \alpha, \ldots, \beta+q \alpha$. And it is well known [8, p. 116] that $2(\hat{\alpha}, \hat{\beta}) /(\hat{\alpha}, \hat{\alpha})=r-q=2\langle\alpha, \beta\rangle /\langle\alpha, \alpha\rangle$.

We remark that Proposition 10 means that the Cartan matrix of $\mathfrak{Q}^{\prime}$, which has the integer $2\left\langle\alpha_{i}, \alpha_{j}\right\rangle\left\langle\left\langle\alpha_{i}, \alpha_{i}\right\rangle\right.$ in the $i j$-position, is contained in the Cartan matrix of $\mathfrak{R}$ in a natural way. It also means that the Dynkin diagram of $\mathfrak{R}^{\prime}$ is embedded in the diagram of $\Re$. Indeed, the diagram of $\Re$ has vertices $\rho_{1}, \ldots, \rho_{r}, \hat{\alpha}_{1}, \ldots, \hat{\alpha}_{l}$; if one deletes the points $\rho_{1}, \ldots, \rho_{r}$ and the lines to these points, one is left with the diagram of $\mathfrak{L}^{\prime}$. As an example, we consider $\mathfrak{S}=\mathfrak{S}\left(\mathfrak{O}_{3}\right)$, the exceptional simple Jordan algebra over $\Phi$. Now if $\Im$ is any simple algebra, dim $\mathfrak{E}=1$. For by $[10$, proof of Theorem 3, p. 314], $\mathfrak{\complement}=\left\{R_{a} \mid a\right.$ is in the center of $\left.\mathfrak{\Im}\right\}$; and the fact that $\Im$ is simple over an algebraically closed field implies that the center of $\mathfrak{\Im}$ is 1-dimensional. For $\Im=S_{\mathcal{C}}\left(\mathfrak{O}_{3}\right), \mathfrak{Q}^{\prime}$ is of type $E_{6}[8$, p. 145] (or [7, p. 37]). Thus $\mathfrak{A}$ is a simple Lie algebra of rank 7 (rank $\mathfrak{\Re}=\operatorname{dim} \mathfrak{C}+\operatorname{dim} \mathfrak{S}_{1}=1+6$ ) whose diagram contains the diagram of $E_{6}$. The only possibility is that $\Re \cong E_{7}$.

We introduce a further piece of notation. If $w \in \operatorname{Hom}_{\Phi}\left(\mathfrak{S}_{1}^{*}, \mathfrak{S}_{1}^{*}\right)$, define $\hat{w}$ to be the unique linear transformation of $\left(\mathfrak{S}_{1}^{*}\right)^{\wedge}$ such that for any $\alpha \in \mathfrak{S}_{1}^{*},(\alpha w)^{\wedge}=\hat{\alpha} \hat{w}$. Clearly $\hat{w}_{1} \hat{w}_{2}=\left(w_{1} w_{2}\right)^{\wedge}$ and $\hat{w}_{1}+\hat{w}_{2}=\left(w_{1}+w_{2}\right)^{\wedge}$.

Proposition 11. If $v$ is in the Weyl group of $\mathfrak{L}^{\prime}$, then $\hat{v}$ can be extended to a unique element $w$ of the Weyl group of $\Re$ having the property that $w$ permutes the roots $\rho$ of $\mathfrak{\AA}$ such that $\rho\left(R_{1}\right)=1$. Conversely, if $w$ is in the Weyl group of $\AA$ and permutes the roots $\rho$ of $\AA$ such that $\rho\left(R_{1}\right)=1$, then $w$ stabilizes $\left(\mathfrak{H}_{1}^{*}\right)^{\wedge}$ and $w \mid\left(\mathfrak{H}_{1}^{*}\right)^{\wedge}=\hat{v}$ for some element $v$ of the Weyl group of $\mathfrak{L}^{\prime}$.

Proof. If $\alpha$ is a root of $\mathfrak{Q}^{\prime}$ and $w_{\alpha}$ is the symmetry of $\mathfrak{S}_{1}^{*}$ in $\alpha$, we show that $\hat{w}_{\alpha}$ $=w_{\alpha} \mid\left(\mathfrak{S}_{1}^{*}\right)^{\wedge}$ and that $w_{\alpha}$ permutes the roots $\rho$ of $\Re$ such that $\rho\left(R_{1}\right)=1$. This will prove the existence of the element $w$ asserted in the first sentence of the proposition; for $v$ is a product of such symmetries. Now for every $\beta \in \mathfrak{S}_{1}^{*}$,

$$
\begin{aligned}
\left(\beta w_{\alpha}\right)^{\wedge} & =\left(\beta-\frac{2\langle\beta, \alpha\rangle}{\langle\alpha, \alpha\rangle} \alpha\right)^{\hat{}}=\hat{\beta}-\frac{2\langle\beta, \alpha\rangle}{\langle\alpha, \alpha\rangle} \hat{\alpha} \\
& =\hat{\beta}-\frac{2(\hat{\beta}, \hat{\alpha})}{(\hat{\alpha}, \hat{\alpha})} \hat{\alpha}=\hat{\beta} w_{\alpha},
\end{aligned}
$$

using Proposition 10. This shows that $w_{\hat{\alpha}} \mid\left(\mathfrak{S}_{1}^{*}\right)^{\wedge}=\left(w_{\alpha}\right)^{\wedge}$. And if $\rho\left(R_{1}\right)=1$, then $\left(\rho w_{\hat{\alpha}}\right)\left(R_{1}\right)=\rho\left(R_{1}\right)-(2(\rho, \hat{\alpha}) /(\hat{\alpha}, \hat{\alpha})) \hat{\alpha}\left(R_{1}\right)=\rho\left(R_{1}\right)-0=1$.

Let $\alpha_{1}, \ldots, \alpha_{l}$ be a simple system of roots of $\mathfrak{R}^{\prime}$ and let $\rho_{1}, \ldots, \rho_{r}, \hat{\alpha}_{1}, \ldots, \hat{\alpha}_{l}$ be the simple system of roots of $\AA$ whose existence was shown in Proposition 9. We note that if $w$ is an element of the Weyl group of $\Re$ which permutes the roots $\rho$ such that $\rho\left(R_{1}\right)=1$ and also permutes $\hat{\alpha}_{1}, \ldots, \hat{\alpha}_{l}$, then $w=1$. For by the Corollary to Proposition 9, $w$ stabilizes the simple system of roots $\left\{\rho_{1}, \ldots, \rho_{r}, \hat{\alpha}_{1}, \ldots, \hat{\alpha}_{l}\right\}$. Now if $w_{1}$ and $w_{2}$ are two extensions of $\hat{v}$ permuting the roots $\rho$ such that $\rho\left(R_{1}\right)=1$, 
then $w_{1} w_{2}^{-1}$ fixes each of the roots $\hat{\alpha}_{1}, \ldots, \hat{\alpha}_{l}$, is in the Weyl group of $\Re$, and permutes the roots $\rho$ such that $\rho\left(R_{1}\right)=1$. Hence $w_{1} w_{2}^{-1}=1$. This proves the uniqueness of the extension of $\hat{v}$.

The same device can be used to prove the converse. First we note that if $w$ is in the Weyl group of $\mathscr{\Omega}$ and permutes the roots $\rho$ such that $\rho\left(R_{1}\right)=1$, then $w$ permutes the roots $\rho$ such that $\rho\left(R_{1}\right)=-1$; indeed, $\rho\left(R_{1}\right)=-1$ implies that $(-\rho)\left(R_{1}\right)=+1$, so that $(\rho w)\left(R_{1}\right)=-((-\rho) w)\left(R_{1}\right)=-1$. But then by Proposition $6(\mathrm{~b}) w$ must also permute the roots $\rho$ such that $\rho\left(R_{1}\right)=0$. These roots span $\left(\mathfrak{S}_{1}^{*}\right)^{\wedge}$, so that $w$ stabilizes $\left(\mathfrak{S}_{1}^{*}\right)^{\wedge}$. To prove the converse, we may then for each $i$ let $\beta_{i}$ be the root of $\mathfrak{Q}^{\prime}$ such that $\hat{\beta}_{i}=\hat{\alpha}_{i} w$; this is possible since $\hat{\alpha}_{i} w$ is a root of $\Re$ and $\left(\hat{\alpha}_{i} w\right)\left(R_{1}\right)=0$-see Proposition $6(\mathrm{c})$. Then $\left\{\beta_{1}, \ldots, \beta_{l}\right\}$ is a simple system of roots of $\mathfrak{Q}^{\prime}$ : it is certainly linearly independent; also if $\beta$ is any root of $\mathfrak{L}^{\prime}$, if $\alpha$ is the root of $\mathfrak{L}^{\prime}$ such that $\hat{\alpha}=\hat{\beta} w^{-1}$, and if $\alpha=\sum n_{i} \alpha_{i}$ (all $n_{i}$ having the same sign), then $\beta=\sum n_{i} \beta_{l}$. Choose $v$ in the Weyl group of $\mathfrak{R}^{\prime}$ so that $\left\{\alpha_{1}, \ldots, \alpha_{l}\right\} v=\left\{\beta_{1}, \ldots, \beta_{l}\right\}$, and let $w_{1}$ be the unique extension of $\hat{v}$ to an element of the Weyl group of $\AA$ permuting the roots $\rho$ such that $\rho\left(R_{1}\right)=1$. Then $w w_{1}^{-1}$ is an element of the Weyl group of $\AA$ permuting the roots $\rho$ such that $\rho\left(R_{1}\right)=1$ and stabilizing the set $\left\{\hat{\alpha}_{1}, \ldots, \hat{\alpha}_{l}\right\}$. Hence $w w_{1}^{-1}=1$ and $w \mid\left(\mathfrak{S}_{1}^{*}\right)^{\wedge}$ $=w_{1} \mid\left(\mathfrak{S}_{1}^{*}\right)^{\wedge}=\hat{v}$.

Since $\varepsilon$ stabilizes both $\mathfrak{S}_{1}$ and $\mathfrak{S}_{2}, \varepsilon^{*}$ permutes the roots of $\mathfrak{L}^{\prime}$ (in its action on $\mathfrak{F}_{1}^{*}$ ) and also permutes the roots of $\mathfrak{R}$ (in its action on $\mathfrak{T}^{*}$ ). In fact, if $\alpha$ is a root of $\mathfrak{R}^{\prime}$ then $\left(\mathfrak{R}_{\alpha}^{\prime}\right) \varepsilon=\mathfrak{L}_{\alpha \varepsilon^{*}-1}^{\prime}=\mathfrak{\Omega}_{\alpha \varepsilon^{*}}^{\prime}$ and similarly if $\rho$ is a root of $\Re_{\text {then }} \mathfrak{R}_{\rho} \varepsilon=\mathfrak{\Re}_{\rho \varepsilon^{*}}$. If $\rho\left(R_{1}\right)=1$, then $\left(\rho \varepsilon^{*}\right)\left(R_{1}\right)=\rho\left(R_{1} \varepsilon\right)=\rho\left(-R_{1}\right)=-1$. Similarly, if $\rho\left(R_{1}\right)=-1$ then $\left(\rho \varepsilon^{*}\right)\left(R_{1}\right)=1$ and if $\rho\left(R_{1}\right)=0$ then $\left(\rho \varepsilon^{*}\right)\left(R_{1}\right)=0$.

Proposition 12. Suppose $v$ is in the Weyl group of $\mathfrak{L}^{\prime}$ and $w$ extends $\hat{v}$ as in Proposition 11. If $v$ commutes with $\varepsilon^{*}$, so does $w$.

Proof. $\varepsilon^{*} w \varepsilon^{*}$ is also in the Weyl group of $\Re$. In fact, if $\rho$ is a root of $\Re$ then $\varepsilon^{*} w_{\rho} \varepsilon^{*}=w_{\rho \varepsilon}$. If $\rho$ is a root of $\Re$ such that $\rho\left(R_{1}\right)=1$, then $-\rho$ is a root and $(-\rho)\left(R_{1}\right)$ $=-1$. So $\left(-\rho \varepsilon^{*}\right)\left(R_{1}\right)=1, \quad\left(-\rho \varepsilon^{*} w\right)\left(R_{1}\right)=1$ and $\left(-\rho \varepsilon^{*} w \varepsilon^{*}\right)\left(R_{1}\right)=-1$. Thus $\varepsilon^{*} w \varepsilon^{*}$ permutes the roots $\rho$ such that $\rho\left(R_{1}\right)=1$. Finally, $\varepsilon^{*} w \varepsilon^{*}$ extends $\hat{v}$ (since $\varepsilon^{*} \mid\left(\mathfrak{S}_{1}^{*}\right)^{\wedge}=\left(\varepsilon^{*}\right)^{\wedge}$, and thus $\left.\varepsilon^{*} w \varepsilon^{*} \mid\left(\mathfrak{S}_{1}^{*}\right)^{\wedge}=\left(\varepsilon^{*}\right)^{\wedge} \hat{v}\left(\varepsilon^{*}\right)^{\wedge}=\left(\varepsilon^{*} v \varepsilon^{*}\right)^{\wedge}=\hat{v}\right)$. The uniqueness assertion of Proposition 11 therefore says that $\varepsilon^{*} w \varepsilon^{*}=w$.

We now investigate the structure of $\mathfrak{A}=\{x \in \mathfrak{S} \mid x \mathfrak{W}=0\}$. Let $P$ be the set of roots $\rho$ of $\Re$ which satisfy $\rho\left(R_{1}\right)=1, \rho(\mathfrak{W})=0$. The first condition means that the root space $\Re_{\rho}$ is contained in $\mathfrak{~}$. If $x \in \mathfrak{\Re}_{\rho}, D \in \mathfrak{W}$ the second condition means that $x D=[x, D]=\rho(D) x=0$. Thus $\mathfrak{R}_{\rho} \subseteq \mathfrak{A}$. In fact, we claim that $\mathfrak{A}=\bigoplus \sum_{\rho \in P} \mathfrak{R}_{\rho}$. First we note that $\mathfrak{A}$ is a subalgebra of $\mathfrak{~}$ : indeed, if $a, b \in \mathfrak{A}$ and $D \in \mathfrak{W}$ then $(a . b) D=$ $a D \cdot b+a \cdot b D=0+0=0$. This means that $\mathfrak{A} \operatorname{ad}_{\mathfrak{A}} \mathfrak{S}=\mathfrak{A} \mathfrak{S}=(\mathfrak{U})\left(R_{\mathfrak{U}}+\mathfrak{W}\right)=\mathfrak{A} \cdot \mathfrak{A}+0 \subseteq \mathfrak{A}$. Since $\mathfrak{A} \cap \mathfrak{Z}=0$ (recall that $\mathfrak{A}$ is a subspace of $\mathfrak{\Im}$, which is embedded in $\mathfrak{T}$ ), we conclude that $\mathfrak{A}$ is a sum of root spaces. If $\rho$ is a root such that $\mathfrak{\Re}_{\rho} \subseteq \mathfrak{A}$, then $\mathfrak{\Re}_{\rho} \mathfrak{W} \subseteq \mathfrak{A} \mathfrak{B}$ $=0$, which shows that $\rho(\mathfrak{W})=0$ and hence that $\rho \in P$. This together with the above means that $\mathfrak{U}=\oplus \sum_{\rho \in P} \mathfrak{R}_{\rho}$. 
Another characterization of $P$ is that $\rho \in P$ if and only if $\rho\left(R_{1}\right)=1, \rho \varepsilon^{*}=-\rho$. Certainly if $\rho(\mathfrak{W})=0$ then for all $R_{a}+D \in \mathfrak{H}, \rho \varepsilon^{*}\left(R_{a}+D\right)=\rho\left(-R_{a}+D\right)=$ $\rho\left(-R_{a}\right)=\rho\left(-R_{a}-D\right)=-\rho\left(R_{a}+D\right)$. Conversely, if $\rho \varepsilon^{*}=-\rho$ then for $D \in \mathfrak{W}$, $\rho(D)=-\rho \varepsilon^{*}(D)=-\rho(D \varepsilon)=-\rho(D)$, so $\rho(D)=0$.

Suppose that $\rho, \rho^{\prime} \in P$ are distinct roots. We show that $\Re_{\rho} \Re_{\rho^{\prime}}=0$, using the fact that $\left[R_{\mathfrak{A}}, R_{\mathfrak{A}}\right]=0$. Let $R_{x}+D \in \mathfrak{S}$ be such that $\rho\left(R_{x}+D\right) \neq \rho^{\prime}\left(R_{x}+D\right)$. Since $\rho(D)$ $=\rho^{\prime}(D)=0$, we have for any $a \in \mathfrak{R}_{\rho}, b \in \mathfrak{R}_{\rho^{\prime}}$

$$
\begin{aligned}
\rho\left(R_{x}+D\right) a \cdot b & =\rho\left(R_{x}\right) a \cdot b=\left(a R_{x}\right) \cdot b=x R_{a} R_{b}=x R_{b} R_{a} \\
& =a \cdot\left(b R_{x}\right)=\rho^{\prime}\left(R_{x}\right) a \cdot b=\rho^{\prime}\left(R_{x}+D\right) a \cdot b,
\end{aligned}
$$

from which we conclude that $a \cdot b=0$.

Proposition 13. (a) $1=\sum_{\rho \in P} e_{\rho}$, where 1 is the identity of $\Im$, each $e_{\rho}$ is a (nonzero) root vector for the root $\rho$, and in addition the $e_{\rho}$ 's are orthogonal idempotents.

(b) Let $\left\{\rho_{1}, \ldots, \rho_{r}, \hat{\alpha}_{1}, \ldots, \hat{\alpha}_{l}\right\}$ be a simple system of roots of $\mathfrak{R}$ as in Proposition 9. Then for each $i$ there exists an element $\alpha$ of $\mathfrak{S}_{1}^{*}$ and $a \rho \in P$ such that $\rho=\rho_{i}+\hat{\alpha}$.

Proof. (a) 1 is certainly a sum $\sum_{\rho} e_{\rho}$ of root vectors, where all the roots $\rho$ appearing in the summation satisfy $\rho\left(R_{1}\right)=1$. If $D \in \mathfrak{B}$ then $0=1 D=\sum_{\rho} \rho(D) e_{\rho}$, i.e. $\rho(D)=0$ for all $\rho$ in the summation. This means that $\rho(\mathfrak{W})=0$ and hence that each $\rho$ in the summation belongs to $P$. The above remarks then show that $e_{\rho} \cdot e_{\rho^{\prime}}=0$ if $\rho \neq \rho^{\prime}$. Multiplying both sides of $1=\sum_{\rho} e_{\rho}$ by $e_{\sigma}$ we find that $e_{\sigma}=e_{\sigma} \cdot e_{\sigma}$, so that the $e_{\rho}$ 's are orthogonal idempotents. Moreover, suppose $\rho \in P$ is such that no root vector for $\rho$ appears in the summation. Let $x$ be a root vector for $\rho$; then $x=1 . x$ $=\sum_{\rho} e_{\rho} \cdot x=0$, a contradiction.

(b) If $1 \leqq i \leqq r$, then $-\rho_{i}\left(R_{1}\right)=-1$, so that $\Re_{-\rho_{1}} \subseteq \Im$. Choose $y \in \Im$ so that $\bar{y}$ is a root vector for $-\rho_{i}$. If $x$ is a root vector for a root $\rho_{j}+\hat{\alpha}, i \neq j, \alpha \in \mathfrak{S}_{1}^{*}$, then $[x, \bar{y}]=0$; this is clear, since $\rho_{j}-\rho_{i}+\hat{\alpha}$ is not a root, by Proposition 8. But every root $\rho$ of $\AA$ such that $\mathfrak{\mathbb { N }}_{\rho} \subseteq \mathfrak{J}$ is of the form $\rho_{j}+\hat{\alpha}$ for some $j$ and some $\alpha$. Thus if no $\rho \in P$ has the form $\rho_{i}+\hat{\alpha}$, we must have

$$
0=\left[\sum_{\rho \in P} e_{\rho}, \bar{y}\right]=[1, \bar{y}]=2\left(R_{1 . y}-\left[R_{1}, R_{y}\right]\right)=2 R_{y},
$$

i.e. $y=0$, a contradiction.

Proposition 14. Let $\left\{\rho_{1}, \ldots, \rho_{r}, \hat{\alpha}_{1}, \ldots, \hat{\alpha}_{l}\right\}$ be as in Proposition 9. Then for each $i$ there exists $\alpha \in \mathfrak{S}_{1}^{*}$ so that $\rho_{i} \varepsilon^{*}=-\rho_{i}-\hat{\alpha}$.

Proof. Choose $\rho \in P$ to have the form $\rho_{i}+\hat{\beta}$ for some $\beta \in \mathfrak{F}_{1}^{*}$; this is possible by Proposition 13(b). Then

$$
\rho_{i} \varepsilon^{*}=(\rho-\hat{\beta}) \varepsilon^{*}=\rho \varepsilon^{*}-\hat{\beta} \varepsilon^{*}=-\rho-\hat{\beta} \varepsilon^{*}=-\rho_{i}-\hat{\beta}-\hat{\beta} \varepsilon^{*} .
$$

Thus we have $\alpha=\beta+\beta \varepsilon^{*}$. 
We next study the connection between roots of $\mathfrak{L}^{\prime}$ and roots of $\mathfrak{D}^{\prime}$. As usual, we define $\mathfrak{S}_{10}^{*}$ to be the rational span in $\mathfrak{S}_{1}^{*}$ of the roots of $\mathfrak{R}^{\prime}$. Then $\operatorname{dim}_{\boldsymbol{Q}} \mathfrak{S}_{10}^{*}=\operatorname{dim}_{\Phi} \mathfrak{S}_{1}^{*}$ and on $\mathfrak{S}_{10}^{*}\langle$,$\rangle is a positive definite (rational) bilinear form. Let \mathfrak{U}_{0}^{*}=\mathfrak{A}^{*} \cap \mathfrak{S}_{10}^{*}$ and $\mathfrak{B}_{0}^{*}=\mathfrak{B}^{*} \cap \mathfrak{S}_{10}^{*}$. We claim that $\mathfrak{S}_{10}^{*}=\mathfrak{A}_{0}^{*}+\mathfrak{W}_{0}^{*}$. Suppose, then, that $\alpha$ is a root of $\mathfrak{I}^{\prime}$. The projection of $\alpha$ on $\mathfrak{A}^{*}$ is $\frac{1}{2}\left(\alpha-\alpha \varepsilon^{*}\right)$ and the projection of $\alpha$ on $\mathfrak{W}^{*}$ is $\frac{1}{2}\left(\alpha+\alpha \varepsilon^{*}\right)$. Since $\alpha \varepsilon^{*}$ is also a root, both projections are in $\mathfrak{S}_{10}^{*}$. Thus, taking rational linear combinations of roots, we see that every element of $\mathfrak{S}_{10}^{*}$ is the sum of an element of $\mathfrak{A}_{0}^{*}$ and one of $\mathfrak{W}_{0}^{*}$.

Note that the projection $\alpha_{-}$of an element $\alpha$ of $\mathfrak{S}_{1}^{*}$ on $\mathfrak{A}^{*}$ is the linear functional which annihilates $\mathfrak{Y}$ and agrees with $\alpha$ on $R_{\mathfrak{U}}$, whereas the projection $\alpha_{+}$of $\alpha$ on $\mathfrak{Y G}^{*}$ is the linear functional which annihilates $R_{\mathfrak{X}^{\prime}}$ and agrees with $\alpha$ on $\mathfrak{M}$. For these two linear functionals are in $\mathfrak{A}^{*}$ and $\mathfrak{W}^{*}$ respectively, and their sum is $\alpha$.

Proposition 15. If $\alpha$ is a root of $\mathfrak{L}^{\prime}$, then $\alpha_{+} \neq 0$. There exists a simple system of roots of $\mathfrak{R}^{\prime}$ which is stabilized by $\varepsilon^{*}$.

Proof. Suppose $\alpha$ is a root of $\mathfrak{Q}^{\prime}$ such that $\alpha(\mathfrak{W})=0$. Let $x \in \mathfrak{I}^{\prime}, D \in \mathscr{D}$ be chosen so that $R_{x}+D$ is a root vector for $\alpha$. By hypothesis, $\left[R_{x}+D, E\right]=0$ for every $E \in \mathfrak{W}$. But $\left[R_{x}+D, E\right]=R_{x E}+[D, E]$, so that $x E=0$ and $[D, E]=0$. This implies that $x \in \mathfrak{A}$ and $D \in \mathfrak{W}$, and hence that $R_{x}+D \in \mathfrak{S}_{1}$, a contradiction.

Thus the projections of all the roots of $\mathfrak{R}^{\prime}$ onto $\mathfrak{W}^{*}$ form a finite collection of nonzero elements of $\mathfrak{W}_{0}^{*}$. We can choose an element $\omega \in \mathfrak{W}_{0}^{*}$ which is not orthogonal to any of these projections. Note that for any $\alpha \in \mathfrak{F}_{1}^{*},\langle\alpha, \omega\rangle=\left\langle\alpha_{+}+\alpha_{-}, \omega\right\rangle$ $=\left\langle\alpha_{+}, \omega\right\rangle$ since $\left\langle\mathfrak{A}^{*}, \mathfrak{B}^{*}\right\rangle=0$. Following [13, p. V-11], we can define a positive system of roots by writing $\alpha>0$ if and only if $\langle\omega, \alpha\rangle>0$. Now if $\alpha>0$, then $\langle\omega, \alpha\rangle>0$, hence $\left\langle\omega \varepsilon^{*}, \alpha \varepsilon^{*}\right\rangle>0$ (since $\varepsilon$ is an automorphism of $\mathfrak{L}^{\prime}, \varepsilon^{*}$ is an orthogonal transformation). But $\omega \varepsilon^{*}=\omega$ and so $\left\langle\omega, \alpha \varepsilon^{*}\right\rangle>0$, i.e. $\left.\alpha \varepsilon^{*}\right\rangle 0$. Thus $\varepsilon^{*}$ maps the positive roots among themselves, and so maps the corresponding simple system into itself.

Proposition 15 is a key result; it allows us to apply a theorem of Steinberg given in [15, Theorem 32, p. 172]. We conclude that $\mathfrak{W}_{0}^{*}$ is a (not necessarily reduced) root space whose roots are the projections of the roots of $\mathfrak{Q}^{\prime}$ onto $\mathfrak{B Z}^{*}$. We call the set of such projections $\Sigma_{\varepsilon}$. The Weyl group of $\Sigma_{\varepsilon}$ is the set of restrictions to $\mathfrak{W}_{0}^{*}$ of elements of the Weyl group of $\mathfrak{Q}^{\prime}$ which commute with $\varepsilon^{*}$. If $w$ is in the Weyl group of $\mathfrak{R}^{\prime}$, commutes with $\varepsilon^{*}$, and satisfies $w \mid \mathfrak{B}_{0}^{*}=1$, then $w=1$. Finally, if $\alpha_{1}, \ldots, \alpha_{l}$ is a simple system of roots of $\mathfrak{R}^{\prime}$ stabilized by $\varepsilon^{*}$, then the set of projections $\alpha_{1+}, \ldots, \alpha_{l+}$ (with duplicates deleted) is a simple system of roots of $\Sigma_{\varepsilon}$.

This is a convenient place to give some well-known facts about three-dimensional simple Jordan algebras.

LEMMA. If $\mathfrak{~ i s ~ a ~ t h r e e - d i m e n s i o n a l ~ s i m p l e ~ J o r d a n ~ a l g e b r a , ~ t h e n ~} \mathfrak{~ i s ~ i s o m o r p h i c ~ t o ~}$ $\Phi 1 \oplus \mathfrak{B}$, the Jordan algebra of a two-dimensional vector space with a nondegenerate bilinear form ( , ). If $v_{1}, v_{2}$ is a basis of $\mathfrak{B}$ with respect to which the form has matrix 
$\left(\begin{array}{ll}0 & 1 \\ 1 & 0\end{array}\right)$, then Der $\Im$ consists of all linear transformations of $\Im$ which annihilate 1 and on $\mathfrak{B}$ have matrix $\left(\begin{array}{ll}\lambda & 0 \\ 0 & -\lambda\end{array}\right), \lambda \in \Phi$.

Proof. The degree of $\mathfrak{\Im}$ (the number of idempotents in a complete set of orthogonal idempotents) must be 2 . If it were 1 then we would have $\mathfrak{\Im}=\Phi 1$. If there were three mutually orthogonal idempotents in $\mathfrak{f}$ we would have $\operatorname{dim} \mathfrak{\Im} \geqq 5$, since the Pierce spaces $\mathfrak{\Im}_{11}, \mathfrak{I}_{22}, \mathfrak{\Im}_{33}, \mathfrak{J}_{12}, \mathfrak{I}_{23}$ would all be nonzero by the simplicity of $\mathfrak{~}$. Thus $\mathfrak{\Im}$ is indeed of the form $\Phi 1 \oplus \mathfrak{B}$, as claimed [10, p. 202].

It is not hard to see that $\Im^{\prime}=\mathfrak{B}$ [10, Exercise 5, p. 14]. Thus if $D$ is a derivation of $\Im, D$ stabilizes $\mathfrak{B}$. If $v_{1} D=\lambda v_{1}+\mu v_{2}$ and $v_{2} D=\lambda^{\prime} v_{1}+\mu^{\prime} v_{2}$, then applying $D$ to both sides of the equations $v_{1} \cdot v_{2}=1$ and $v_{i}^{2}=0(i=1,2)$, we find that $v_{1} D \cdot v_{2}+v_{2} D \cdot v_{1}$ $=0=v_{1} \cdot v_{1} D=v_{2} \cdot v_{2} D$, and hence that $\lambda+\mu^{\prime}=0=\mu=\lambda^{\prime}$. This shows that $v_{1} D$ $=\lambda v_{1}, v_{2} D=-\lambda v_{2}$ as desired. Conversely, such a linear transformation is indeed a derivation, since it satisfies $v_{1} D \cdot v_{2}+v_{2} D \cdot v_{1}=\left(v_{1} \cdot v_{2}\right) D$ and $2 v_{i} \cdot v_{i} D=v_{i}^{2} D(i=1,2)$.

Proposition 16. Let $\alpha$ be a root of $\mathfrak{L}^{\prime}$ such that $\alpha(\mathbb{E}) \neq 0$. Then a root vector of $\alpha$ has the form $R_{a}$ where a belongs to a three-dimensional simple ideal of $\Im$, and where $a \mathfrak{D}^{\prime}=0$. The only roots $\beta$ of $\mathfrak{L}^{\prime}$ such that $\langle\beta, \alpha\rangle \neq 0$ are $\pm \alpha$.

Proof. Let $\mathfrak{\Im}=\oplus \sum_{i} \mathfrak{\Im}_{i}$, where $\mathfrak{\Im}_{i}$ are simple ideals of $\mathfrak{\Im}$. Then $\mathfrak{L}=\oplus \sum_{i} \mathfrak{L}\left(\mathfrak{\Im}_{i}\right)$ and hence $\mathfrak{L}^{\prime}=\oplus \sum_{i} \mathfrak{L}\left(\mathfrak{\Im}_{i}\right)^{\prime}$. Each root space of $\mathfrak{L}^{\prime}$ is contained in some $\mathfrak{L}\left(\mathfrak{\Im}_{i}\right)^{\prime}$-say $\mathfrak{R}_{\alpha}^{\prime} \subseteq \mathfrak{Q}\left(\mathfrak{\Im}_{i}\right)^{\prime}$. Two roots whose root spaces are contained in different summands $\mathfrak{L}\left(\mathfrak{J}_{1}\right)^{\prime}$ are orthogonal. Now $\mathfrak{E}=\oplus \sum_{i} \mathfrak{E}\left(\mathfrak{\Im}_{i}\right)$, where $\mathfrak{E}\left(\mathfrak{\Im}_{i}\right)$ is the center of Der $\mathfrak{\Im}_{i}$. Since $\left[\mathfrak{R}_{\alpha}^{\prime}, \mathfrak{E}\right] \neq 0, \mathfrak{E}\left(\mathfrak{\Im}_{1}\right) \neq 0$. Now the derivation algebra of any simple Jordan algebra is semisimple unless the Jordan algebra is three-dimensional (see Helwig [4] for a proof which does not use the classification theory of simple Jordan algebras; in general, with a few low-dimensional exceptions, the derivation algebra of a simple Jordan algebra over $\Phi$ is in fact simple [6]). Thus $\Im_{1}$ is three-dimensional. Let $R_{a}+B$ be a root vector for $\alpha$ and let $E \in \mathbb{E}$ be chosen so that $\alpha(E) \neq 0$. Then $\alpha(E) R_{a}+\alpha(E) B=\alpha(E)\left(R_{a}+B\right)=\left[R_{a}+B, E\right]=R_{a E}+[B, E]=R_{\alpha E}$, which means that $B=0$. Thus $R_{a} \in \mathfrak{L}\left(\Im_{1}\right)^{\prime}$ and so $a \in \Im_{1}$.

$\mathfrak{W} \cap \operatorname{Der} \Im_{1}$ is a Cartan subalgebra of Der $\Im_{1}$, which is one-dimensional abelian. Hence $\mathfrak{W} \cap \operatorname{Der} \mathfrak{\Im}_{1}=$ Der $\mathfrak{\Im}_{1}$. The lemma on three-dimensional algebras shows that $\mathfrak{U} \cap \mathfrak{\Im}_{1}=\left\{x \in \mathfrak{\Im}_{1} \mid x\right.$ Der $\left.\mathfrak{\Im}_{1}=0\right\}=\Phi e_{1}$, where $e_{1}$ is the identity of $\mathfrak{\Im}_{1}$. Now $\operatorname{dim} \mathfrak{Q}\left(\mathfrak{\Im}_{1}\right)$ $=\operatorname{dim} \mathfrak{I}_{1}+\operatorname{dim} \operatorname{Der} \mathfrak{\Im}_{1}=3+1=4$. Also $\mathfrak{L}\left(\mathfrak{\Im}_{1}\right)$ is the direct sum of the root spaces of roots $\beta$ such that $\mathfrak{R}_{\beta}^{\prime} \subseteq \mathfrak{L}\left(\mathfrak{I}_{1}\right)^{\prime}$ and $\mathfrak{K} \cap \mathfrak{L}\left(\mathfrak{I}_{1}\right)=R_{\mathfrak{U} \cap \mathfrak{I}_{1}} \oplus\left(\mathfrak{W} \cap\right.$ Der $\left.\mathfrak{I}_{1}\right)=\Phi R_{e_{1}}$ $+\operatorname{Der} \Im_{1}$, which is two-dimensional. Hence there are exactly two such roots $\beta$, which must be $\pm \alpha$. Finally, since Der $\Im_{1}$ is abelian, $\mathfrak{D}^{\prime} \subseteq \bigoplus \sum_{i \neq 1} \operatorname{Der}\left(\Im_{1}\right)$. Hence $a \in \Im_{1}$ implies that $a \mathfrak{D}^{\prime}=0$.

COROLlaRY. For any root $\alpha$ of $\mathfrak{R}^{\prime}$, either $\alpha(\mathfrak{E})=0$ or $\alpha\left(\mathfrak{W}_{1}\right)=0$.

Proof. If $\alpha(\mathscr{E}) \neq 0$, then let $R_{a}$ be a root vector for $\alpha$ as above. $\left[R_{\alpha}, \mathfrak{W}_{1}\right]=R_{a \mathfrak{W}_{1}}$ $\subseteq R_{a \mathfrak{D}^{\prime}}=0$. 
This corollary shows that if $\alpha$ is a root of $\mathfrak{R}^{\prime}$, then the projection $\alpha_{+}$of $\alpha$ onto $\mathfrak{W}^{*}$ belongs either to $\mathfrak{F}^{*}$ or to $\left(\mathfrak{W}_{1}^{*}\right)^{-}$. Let $\mathfrak{F}_{0}^{*}=\mathfrak{W}_{0}^{*} \cap \mathfrak{F}^{*}$ and $\left(\mathfrak{W}_{1}^{*}\right)_{0}^{-}=\mathfrak{W}_{0}^{*} \cap\left(\mathfrak{W}_{1}^{*}\right)^{-}$. Since $\mathfrak{W}_{0}^{*}$ is the rational span of the projections onto $\mathfrak{W}^{*}$ of the roots of $\mathfrak{L}^{\prime}$, it follows that $\mathfrak{W}_{0}^{*}=\mathfrak{E}_{0}^{*}+\left(\mathfrak{W}_{1}^{*}\right)_{0}^{-}$.

Proposition 17. Let $\alpha$ be a root of $\mathfrak{R}^{\prime}$ and let $R_{a}+B$ be a root vector for $\alpha$. If $B \neq 0$, then $\alpha \mid \mathfrak{W}_{1}$ is a root of $\mathfrak{D}^{\prime}$ with root vector $B$.

Proof. If $D \in \mathfrak{W}_{1}$ then $\alpha(D) R_{a}+\alpha(D) B=\left[R_{a}+B, D\right]=R_{a D}+[B, D]$. Equating terms we see that $[B, D]=\alpha(D) B$. We need to show that $\alpha\left(\mathfrak{W}_{1}\right) \neq 0$, for this will show that $B \in \mathfrak{D}^{\prime}$ and that $\alpha$ is a root of $\mathfrak{D}^{\prime}$. Now if $\alpha\left(\mathfrak{W}_{1}\right)$ were zero, then since $\alpha(\mathfrak{W}) \neq 0$ (by Proposition 15) we would have $\alpha(\mathfrak{E}) \neq 0$. Choose $E \in \mathbb{E}$ so that $\alpha(E)$ $\neq 0$. Then $0=[B, E]=\alpha(E) B$ and hence $B=0$, a contradiction.

Proposition 18. (a) If $\alpha$ and $\omega$ are roots of $\mathfrak{R}^{\prime}$ with $\alpha_{+}=\omega$, then $\alpha=\omega$.

(b) If $\alpha$ and $\beta$ are roots of $\mathfrak{R}^{\prime}$ with $\alpha_{+}=\beta_{+}$, then either $\alpha=\beta$ or $\alpha=\beta \varepsilon^{*}$.

Proof. (a) If $\alpha \neq \omega$ then $\alpha_{-} \neq 0$. We show that this is impossible. Now

$$
\frac{2\langle\alpha, \omega\rangle}{\langle\omega, \omega\rangle}=\frac{2\left\langle\alpha_{-}+\omega, \omega\right\rangle}{\left\langle\alpha_{-}+\omega, \alpha_{-}+\omega\right\rangle}=\frac{2\langle\omega, \omega\rangle}{\left\langle\alpha_{-}, \alpha_{-}\right\rangle+\langle\omega, \omega\rangle}=\frac{2}{\left(\left\langle\alpha_{-}, \alpha_{-}\right\rangle /\langle\omega, \omega\rangle\right)+1} .
$$

Since $0 \neq \alpha_{-} \in \mathfrak{S}_{10}^{*}$, we have $\left\langle\alpha_{-}, \alpha_{-}\right\rangle>0$. This implies that $2\langle\alpha, \omega\rangle \mid\langle\omega, \omega\rangle$ is bigger than zero and less than two. But $2\langle\alpha, \omega\rangle /\langle\omega, \omega\rangle$ is an integer, and so must be equal to one. Now if $w_{\alpha}$ is the symmetry of $\mathfrak{H}_{1}^{*}$ in $\alpha$, then $\omega w_{\alpha}$ is a root of $\mathfrak{\Omega}^{\prime}$. And $\omega w_{\alpha}=\omega-(2\langle\alpha, \omega\rangle \mid\langle\omega, \omega\rangle) \alpha=\omega-\alpha=-\alpha_{-}$. But $-\alpha_{-} \in \mathfrak{Y}_{0}^{*}$, contradicting Proposition 15.

(b) If $\alpha \in \mathfrak{W}^{*}$, then $\alpha=\alpha_{+}$and $\alpha=\beta$ by part (a). Similarly if $\beta \in \mathfrak{W}^{*}$ then $\alpha=\beta$. So suppose $\alpha\left(R_{\mathfrak{A}^{\prime}}\right) \neq 0 \neq \beta\left(R_{\mathfrak{U}^{\prime}}\right)$. Choose $R_{x} \in R_{\mathfrak{U}^{\prime}}$ to be a common nonzero of $\alpha$ and $\beta$. Let $R_{a}+B$ be a root vector for $\alpha$ and $R_{c}+D$ a root vector for $\beta$. $B$ is not zero: otherwise $\alpha\left(R_{x}\right) R_{a}=\left[R_{a}, R_{x}\right]$ and hence $\alpha\left(R_{x}\right)=0$. Similarly $D \neq 0$. So by Proposition $17, B$ and $D$ are root vectors for the $\operatorname{root} \alpha\left|\mathfrak{W}_{1}=\alpha_{+}\right| \mathfrak{W}_{1}=\beta_{+}\left|\mathfrak{W}_{1}=\beta\right| \mathfrak{W}_{1}$. Since root spaces of $\mathfrak{D}^{\prime}$ are one-dimensional, we can multiply by a scalar and assume $B=D$. Then $\alpha\left(R_{x}\right)\left(R_{a}+B\right)=\left[R_{a}+B, R_{x}\right]=-R_{x B}+\left[R_{a}, R_{x}\right]$, i.e. $\alpha\left(R_{x}\right) R_{a}=-R_{x B}$. We conclude that $a=-\alpha\left(R_{x}\right)^{-1} x B$ and similarly that $c=-\beta\left(R_{x}\right)^{-1} x B$. If $x B=0$ then $a=c=0$ and $\alpha=\beta$. If $x B \neq 0$ then $c$ is a scalar multiple of $a$. So the root space $\mathfrak{R}_{\beta}^{\prime}$ is contained in $\Phi R_{a}+\Phi B=\Phi\left(R_{a}+B\right)+\Phi\left(-R_{a}+B\right)=\mathfrak{R}_{\alpha}^{\prime}+\mathfrak{R}_{\alpha \varepsilon}^{\prime}$. Hence $\beta=\alpha$ or $\beta=\alpha \varepsilon^{*}$.

Proposition 19. (a) Let $\omega$ be a root of $\mathfrak{D}^{\prime}$. Then there exists a root $\beta$ of $\mathfrak{L}^{\prime}$ with root vector $R_{a}+B, B \neq 0$, such that $\beta \mid \mathfrak{W}_{1}=\omega$.

(b) Let $\omega$ be a root of $\mathfrak{L}^{\prime}$ such that $\omega \mid \mathfrak{W}_{1}$ is a root of $\mathfrak{D}^{\prime}$. Then a root vector for $\omega$ has the form $R_{a}+B, B \neq 0$.

Proof. (a) Let $B$ be a root vector for $\omega$. Since $\mathfrak{L}^{\prime}$ is the direct sum of its root spaces and $\mathfrak{S}_{1}$, we have $B=H+\sum_{\alpha}\left(R_{a_{\alpha}}+B_{\alpha}\right)$, where $H$ is some element of $\mathfrak{S}_{1}$, 
where the sum is over certain roots $\alpha$ of $\mathfrak{L}^{\prime}$, and where $R_{a_{\alpha}}+B_{\alpha}$ is some (nonzero) root vector of $\alpha$. If $D \in \mathfrak{W}_{1}$, we have $\omega(D) B=[B, D]=0+\sum_{\alpha} \alpha(D)\left(R_{a_{\alpha}}+B_{\alpha}\right)$. Choosing $D$ so that $\omega(D) \neq 0$, we see that $B=\omega(D)^{-1} \sum_{\alpha} \alpha(D)\left(R_{a_{\alpha}}+B_{\alpha}\right)$ and hence that $H=0$. Also we see that $\alpha(D) / \omega(D)=1$ for every $\alpha$ appearing in the summation. This means that $\alpha$ and $\omega$ agree on the Zariski open subset of $\mathfrak{W}_{1}$ consisting of the nonzeroes of $\omega$, i.e. $\alpha \mid \mathfrak{W}_{1}=\omega$. And certainly by the Corollary to Proposition 16, $\alpha(\mathcal{E})=0$. So if $\alpha$ and $\beta$ are any two roots which occur in the summation, $\alpha|\mathfrak{W}=\beta| \mathfrak{W}$; by Proposition 18(b), $\alpha=\beta$ or $\alpha=\beta \varepsilon^{*}$. Thus there are at most two roots in the summation. If there is only one, say $B=R_{a_{\alpha}}+B_{\alpha}$, then $R_{a_{\alpha}}=0$ and $B_{\alpha} \neq 0$. If there are two, say $B=R_{a_{\alpha}}+B_{\alpha}+\lambda\left(-R_{a_{\alpha}}+B_{\alpha}\right), \lambda \in \Phi$, then we must have $\lambda=1$, $B=2 B_{\alpha} \neq 0$.

(b) Suppose the contrary. Then $\omega$ has a root vector $R_{a}$. If $x \in \mathfrak{A}^{\prime}$ then $\left[R_{a}, R_{x}\right]=$ $\omega\left(R_{x}\right) R_{a}$ and hence $\omega\left(R_{x}\right)=0$. So $\omega\left(R_{\mathfrak{A}}\right)=0$, i.e. $\omega \in \mathfrak{W}^{*}$. Now by part (a), there is a root $\beta$ of $\mathfrak{Q}^{\prime}$ with root vector $R_{b}+B, B \neq 0$, so that $\beta\left|\mathfrak{W}_{1}=\omega\right| \mathfrak{W}_{1}$. We claim that the projection of $\beta$ on $\mathfrak{W}^{*}$ is $\omega$. Indeed, since $\beta$ and $\omega$ agree on $\mathfrak{W}_{1}$, we only have to show they agree on $\mathbb{E}$. But since $\omega\left(\mathfrak{W}_{1}\right) \neq 0 \neq \beta\left(\mathfrak{W}_{1}\right)$, we have $\omega(\mathfrak{E})=\beta(\mathfrak{E})=0$ by the Corollary to Proposition 16. Thus $\beta_{+}=\omega_{+}$, and so by Proposition 18(b) $\omega=\beta$ or $\omega=\beta \varepsilon^{*}$. Hence a root vector for $\omega$ must be proportional either to $R_{b}+B$ or to $-R_{b}+B, B \neq 0$. Clearly $R_{a}$ does not have this property, a contradiction.

Suppose $\omega$ is a root of $\mathfrak{D}^{\prime}$. Then $\bar{\omega}$, the linear functional on $\mathfrak{S}_{1}$ which annihilates $R_{\mathfrak{A}^{\prime}}$ and $\mathfrak{E}$ and agrees with $\omega$ on $\mathfrak{W}_{1}$, is the projection on $\mathfrak{W}^{*}$ of some root $\alpha$ of $\mathfrak{L}^{\prime}$, by Proposition 19(a). In particular, $\bar{\omega} \in\left(\mathfrak{W}_{1}^{*}\right)_{0}^{-}$. Let $\mathfrak{W}_{10}^{*}$ be the rational span in $\mathfrak{W}_{1}^{*}$ of the roots of $\mathfrak{D}^{\prime}$. Then $\left(\mathfrak{B}_{10}^{*}\right)^{-} \subseteq\left(\mathfrak{W}_{1}^{*}\right)_{0}^{-}$. It is well known that $\operatorname{dim}_{\mathcal{Q}} \mathfrak{W}_{10}^{*}$ $=\operatorname{dim}_{\Phi} \mathfrak{W}_{1}^{*}$. Also $\operatorname{dim}_{\Phi} \mathfrak{A}^{*}+\operatorname{dim}_{\Phi} \mathfrak{F}^{*}+\operatorname{dim}_{\Phi}\left(\mathfrak{W}_{1}^{*}\right)^{-}=\operatorname{dim}_{\Phi} \mathfrak{H}_{1}^{*}=\operatorname{dim}_{Q} \mathfrak{H}_{10}^{*}=\operatorname{dim}_{Q} \mathfrak{A}_{0}^{*}$ $+\operatorname{dim}_{Q} \mathfrak{F}_{0}^{*}+\operatorname{dim}_{Q}\left(\mathfrak{W}_{1}^{*}\right)_{0}^{-}$. Since $\operatorname{dim}_{\Phi} \mathfrak{A}^{*} \leqq \operatorname{dim}_{\boldsymbol{Q}} \mathfrak{H}_{0}^{*}, \operatorname{dim}_{\Phi} \mathfrak{F}^{*} \leqq \operatorname{dim}_{\boldsymbol{Q}} \mathfrak{F}_{0}^{*}$ and $\operatorname{dim}_{\Phi}\left(\mathfrak{W}_{1}^{*}\right)^{-} \leqq \operatorname{dim}_{Q}\left(\mathfrak{W}_{1}^{*}\right)_{0}^{-}$, equality holds in these three inequalities. In particular, $\operatorname{dim}_{Q}\left(\mathfrak{W}_{1}^{*}\right)_{0}^{-}=\operatorname{dim}_{\Phi} \mathfrak{W}_{1}^{*}$. Since $\omega \mapsto \bar{\omega}$ is injective, we must have $\left(\mathfrak{W}_{10}^{*}\right)^{-}=\left(\mathfrak{W}_{1}^{*}\right)_{0}^{-}$.

3. Automorphisms. In this section we use the root systems defined above to study the groups $G$ and $\Gamma$. First, however, we recall some facts about automorphisms of Lie algebras which will be needed below. Let $\mathfrak{L}$ be a semisimple Lie algebra over $\Phi, \mathfrak{F}$ a Cartan subalgebra of $\mathfrak{L},\left\{\alpha_{1}, \ldots, \alpha_{l}\right\}$ a simple system of roots of $\mathfrak{L}$, and $\left\{e_{i}, f_{i}, h_{i} \mid i=1, \ldots, l\right\}$ a canonical system of generators for $\mathfrak{L}[8$, p. 275]. We wish to describe the group of automorphisms of $\mathfrak{L}$ fixing $\mathfrak{S}$ pointwise. Any automorphism of $\mathfrak{Q}$ is determined by its action on $e_{i}$ and $f_{i}, i=1, \ldots, l$. An automorphism $\eta$ of $\mathfrak{L}$ which fixes $\mathfrak{S}$ pointwise stabilizes the root spaces of $\mathfrak{L}$; in particular, there exist nonzero scalars $\theta_{1}, \ldots, \theta_{l}$ such that $e_{i} \eta=\theta_{i} e_{i}$ for all $i$. Since $\left[e_{i}, f_{i}\right]=h_{i}$ and $h_{i} \eta=1$, it follows that $f_{i} \eta=\theta_{i}^{-1} f_{i}$. An induction on the level of roots shows that on the root space of the root $\sum_{i} n_{i} \alpha_{i}, \eta$ is scalar multiplication by $\theta_{1}^{n_{1}} \cdots \theta_{l}^{n_{l}}$. Conversely, if $\theta_{1}, \ldots, \theta_{l}$ is any sequence of nonzero scalars, then there exists a unique automorphism $\eta$ of $\mathfrak{R}$ such that $e_{i} \eta=\theta_{i} e_{i}, f_{i} \eta=\theta_{i}^{-1} f_{i}$, and $h_{i} \eta=h_{i}(i=1, \ldots, l)$. Indeed, $\left\{\theta_{i} e_{i}, \theta_{i}^{-1} f_{i}, h_{i} \mid i=1, \ldots, l\right\}$ is also a canonical system of generators of $\mathfrak{L}$, and so $\eta$ 
exists by the standard isomorphism theorem [8, p. 127]. The mapping $\left(\theta_{1}, \ldots, \theta_{l}\right)$ $\mapsto \eta$ is thus a birational isomorphism of the $l$-dimensional torus (the direct product of the multiplicative group of $\Phi$ taken $l$ times) onto the group of automorphisms of $\mathfrak{L}$ fixing $\mathfrak{S}$ pointwise; we conclude that the latter group is an irreducible linear group.

We recall that an invariant automorphism of a Lie algebra $\mathfrak{L}$ is a product of automorphisms of the form $\exp \left(\operatorname{ad}_{\mathfrak{L}} L\right)$, where $L \in \mathfrak{Q}$ is such that $\operatorname{ad}_{\mathfrak{L}} L$ is nilpotent. We now return to the study of the Jordan algebra $\Im$ and the groups $G$ and $\Gamma$.

Proposition 20. Any invariant automorphism of $\mathfrak{L}^{\prime}$ can be extended to an element of $\tilde{\Gamma}_{0}$.

Proof. We need only show that automorphisms of the form $\exp \left(\operatorname{ad}_{\mathfrak{R}^{\prime}} L\right)$, where $L \in \mathfrak{R}^{\prime}$ and $\operatorname{ad}_{\mathfrak{R}^{\prime}} L$ is nilpotent, can be extended. Now by [8, p. 100], there exists $H \in \mathfrak{R}^{\prime}$ such that $\left[\operatorname{ad}_{\mathfrak{L}^{\prime}} L, \operatorname{ad}_{\mathfrak{L}^{\prime}} H\right]=2 \operatorname{ad} \mathfrak{Q}^{\prime} L$. Since $\mathfrak{L}^{\prime}$ is semisimple the adjoint representation is faithful and $[L, H]=2 L$. But then $\left[\operatorname{ad}_{\mathcal{R}} L, \operatorname{ad}_{\Re} H\right]=2 \operatorname{ad}_{\mathcal{R}} L$, which shows that $\operatorname{ad}_{\Re} L$ is nilpotent [8, p. 44]. Thus $\exp \left(\operatorname{ad}_{\Re} L\right)$ is an automorphism of $\Re$, which clearly extends $\exp \left(\operatorname{ad}_{\mathfrak{R}^{\prime}} L\right)$. It fixes $R_{1}$ since $\left[R_{1}, L\right]=0$, and hence is in $\tilde{\Gamma}$ by Proposition 4. Moreover, $\left\{\exp \operatorname{ad}_{\mathcal{A}} \lambda L \mid \lambda \in \Phi\right\}$ is an irreducible set of automorphisms of $\mathfrak{R}$; for $\lambda \mapsto \exp \operatorname{ad}_{\mathcal{R}} \lambda L$ is a polynomial map from $\Phi$, an irreducible set, to $\tilde{\Gamma}$. This set contains $\exp \operatorname{ad}_{\mathcal{R}} 0 \cdot L=1$ and so must be entirely contained in $\tilde{\Gamma}_{0}$. In particular, $\exp \operatorname{ad}_{\Re} L \in \tilde{\Gamma}_{0}$.

THEOREM 1. (a) If $\eta \in \tilde{\Gamma}$, there exists a $\tau \in \tilde{\Gamma}_{0}$ so that $\eta \tau$ stabilizes $\mathfrak{H}$.

(b) If $\eta \in \tilde{\Gamma}$ stabilizes $\mathfrak{S}$, then $\eta^{*}$ (acting on $\mathfrak{S}^{*}$ ) permutes the roots of $\mathfrak{\Omega}$ and permutes the roots $\rho$ such that $\rho\left(R_{1}\right)=1$. Conversely, if $\eta \in$ Aut $\mathfrak{\Re}$ stabilizes $\mathfrak{S}$ and if $\eta^{*}$ permutes the roots $\rho$ of $\Re$ such that $\rho\left(R_{1}\right)=1$, then $\eta \in \tilde{\Gamma}$.

(c) If $\eta \in \tilde{\Gamma}$ stabilizes $\mathfrak{H}$, then $\eta \in \tilde{\Gamma}_{0}$ if and only if $\eta^{*}$ is in the Weyl group of $\mathfrak{\Omega}$.

Proof. (a) Since $\eta \in \tilde{\Gamma}, \eta$ stabilizes $\mathfrak{L}$, and hence also stabilizes $\mathfrak{L}^{\prime}$ and $\mathfrak{E}$. Let $\mathfrak{S}_{2}=\mathfrak{S}_{1} \eta$; here $\mathfrak{S}_{2}$ is also a Cartan subalgebra of $\mathfrak{L}^{\prime}$. By [8, p. 273] there is an invariant automorphism $\tau_{1}$ of $\mathfrak{R}^{\prime}$ such that $\mathfrak{S}_{2} \tau_{1}=\mathfrak{S}_{1}$. Extend $\tau_{1}$ to $\tau \in \tilde{\Gamma}_{0}$ as in Proposition 20. Thus $\mathfrak{S}_{1} \eta \tau=\mathfrak{S}_{1}$. Certainly $\mathfrak{C}_{\eta \tau} \subseteq \mathfrak{S}$ (since $\eta \tau \in \tilde{\Gamma}$ ) and thus $\mathfrak{S} \eta \tau=\mathfrak{H}$.

(b) Since $\eta$ stabilizes $\mathfrak{S}$ then $\eta^{*}$ permutes the roots of $\mathfrak{A}$ : in fact, if $\rho$ is a root of $\mathfrak{\Re}$, then $\mathfrak{R}_{\rho} \eta^{-1}=\mathfrak{R}_{\rho \eta^{*}}$ [8, p. 276]. If $\rho\left(R_{1}\right)=1$, then by Proposition 6(b) $\mathfrak{R}_{\rho} \subseteq \Im$. This means that $\mathfrak{N}_{\rho \eta^{*}}=\mathfrak{R}_{\rho} \eta^{-1} \subseteq \mathfrak{J}$ and hence that $\left(\rho \eta^{*}\right)\left(R_{1}\right)=1$, again by Proposition 6(b).

To prove the converse, we note that if $\eta^{*}$ permutes the roots $\rho$ such that $\rho\left(R_{1}\right)=1$, it also permutes the roots $\rho$ such that $\rho\left(R_{1}\right)=-1$ and the roots $\rho$ such that $\rho\left(R_{1}\right)=0$ (the argument for this appears at the beginning of the third paragraph of the proof of Proposition 11). In summary, $\left(\rho \eta^{*}\right)\left(R_{1}\right)=\rho\left(R_{1}\right)$ for every root $\rho$ of $\Re$. Since the roots of $\mathfrak{R}$ span $\mathfrak{S}^{*}$, we see that $\rho\left(R_{1} \eta\right)=\left(\rho \eta^{*}\right)\left(R_{1}\right)=\rho\left(R_{1}\right)$ for every $\rho \in \mathfrak{S}^{*}$. This certainly means that $R_{1} \eta=R_{1}$, and hence by Proposition 4 that $\eta \in \tilde{\Gamma}$. 
(c) Suppose $\eta \in \tilde{\Gamma}_{0}$. Then in particular $\eta \in$ Aut $_{0} \Re$, which means (by the results on Lie algebras described in the Introduction) that $\eta^{*}$ is in the Weyl group of $\mathfrak{R}$.

Conversely, suppose that $\eta^{*}$ is in the Weyl group of $\Re$. Since $\rho\left(R_{1}\right)=1$ implies $\left(\rho \eta^{*}\right)\left(R_{1}\right)=1$, the second part of Proposition 11 implies that $\eta^{*}$ stabilizes $\left(\mathfrak{S}_{1}^{*}\right)^{\wedge}$ and that $\eta^{*} \mid\left(\mathfrak{S}_{1}^{*}\right)^{\wedge}=\hat{v}$, where $v$ is in the Weyl group of $\mathfrak{L}^{\prime}$. By [8, p. 276] there exists an invariant automorphism $\tau_{1}$ of $\mathfrak{L}^{\prime}$ which stabilizes $\mathfrak{H}_{1}$ and which satisfies $\tau_{1}^{*}=v$ (in its action on $\mathfrak{H}_{1}^{*}$ ). Extend $\tau_{1}$ to $\tau \in \tilde{\Gamma}_{0}$, using Proposition 20. Since $\mathfrak{L} \tau \subseteq \mathfrak{L}$ and hence $\mathfrak{夭} \tau \subseteq \mathfrak{C}, \tau$ stabilizes $\mathfrak{H}_{\mathrm{e}}$. Also $\tau^{*} \mid\left(\mathfrak{H}_{1}^{*}\right)^{\wedge}=\left(\tau_{1}^{*}\right)^{\wedge}$; for if $C \in \mathfrak{C}$, $H \in \mathfrak{S}_{1}$, and $\alpha \in \mathfrak{S}_{1}^{*}$ then $\hat{\alpha}\left(\tau_{1}^{*}\right)^{\wedge}(C+H)=\left(\alpha \tau_{1}^{*}\right)^{\wedge}(C+H)=\alpha \tau_{1}^{*}(H)=\alpha\left(H \tau_{1}\right)=\alpha(H \tau)$ $=\hat{\alpha}(C \tau+H \tau)=\hat{\alpha}((C+H) \tau)=\hat{\alpha} \tau^{*}(C+H)$. By the first part of the proof of $(\mathrm{c}), \tau^{*}$ is in the Weyl group of $\Re$. Certainly $\tau^{*}$ permutes the roots $\rho$ of $\Re$ such that $\rho\left(R_{1}\right)=1$. The uniqueness assertion in the first part of Proposition 11 then shows that $\tau^{*}=\eta^{*}$.

Consequently $\left(\eta \tau^{-1}\right)^{*}=1$, which means that $\eta \tau^{-1}$ fixes pointwise. At the beginning of this section we showed that the group of automorphisms of $\mathfrak{A}$ fixing $\mathfrak{F}$ pointwise is irreducible; this group is contained in $\tilde{\Gamma}$ since in particular $R_{1} \in \mathfrak{F}_{\mathfrak{E}}$ (using Proposition 4), and hence is contained in $\tilde{\Gamma}_{0}$. We conclude that $\eta \tau^{-1} \in \tilde{\Gamma}_{0}$, i.e. that $\eta \in \tilde{\Gamma}_{0} \tau=\tilde{\Gamma}_{0}\left(\right.$ since $\left.\tau \in \tilde{\Gamma}_{0}\right)$.

COROllaRY. Let $\rho_{1}, \ldots, \rho_{r}, \hat{\alpha}_{1}, \ldots, \hat{\alpha}_{l}$ be a simple system of roots of $\mathfrak{\AA}$ as in Proposition 9. If $\eta \in \tilde{\Gamma}$, then there exists $\tau \in \tilde{\Gamma}_{0}$ such that $\eta \tau$ stabilizes $\mathfrak{S}$ and $(\eta \tau)^{*}$ stabilizes the given simple system.

Proof. Choose $\tau_{1} \in \tilde{\Gamma}_{0}$ so that $\eta_{1}=\eta \tau_{1}$ stabilizes $\mathfrak{S}$ (using Theorem 1(a)). Then $\eta_{1} \in \tilde{\Gamma}$ and so $\eta_{1}^{*}$ permutes the roots $\hat{\alpha}$ of $\Re$ (where $\alpha$ is a root of $\mathfrak{Q}^{\prime}$ ); for they are the roots of $\mathfrak{\AA}$ which annihilate $R_{1}$. Thus $\hat{\alpha}_{i} \eta^{*-1}=\hat{\beta}_{i}$ for some root $\beta_{l}$ of $\mathfrak{L}^{\prime}$. Now $\left\{\beta_{1}, \ldots, \beta_{l}\right\}$ must be a simple system of roots of $\mathfrak{L}^{\prime}$. For if $\alpha$ is any root of $\mathfrak{L}^{\prime}, \hat{\alpha} \eta_{1}^{*}=\hat{\beta}$ ( $\beta$ some root of $\mathfrak{L}^{\prime}$ ). There exist integers $n_{1}, \ldots, n_{l}$ of the same sign such that $\beta=\sum_{i} n_{i} \alpha_{i}$; then $\hat{\alpha}=\hat{\beta} \eta_{1}^{*-1}=\sum_{i} n_{i} \hat{\alpha}_{i} \eta_{1}^{*-1}=\sum_{i} n_{i} \beta_{i}$.

Let $v$ be the element of the Weyl group of $\mathfrak{L}^{\prime}$ which maps $\left\{\alpha_{1}, \ldots, \alpha_{l}\right\}$ to $\left\{\beta_{1}, \ldots, \beta_{l}\right\}$. Let $w$ in the Weyl group of $\AA$ extend $\hat{v}$, as in Proposition 11. Then $w \eta_{1}^{*}$ permutes $\left\{\hat{\alpha}_{1}, \ldots, \hat{\alpha}_{l}\right\}$, hence by the Corollary to Proposition 9 permutes $\left\{\dot{\rho}_{1}, \ldots, \rho_{r^{\prime}}, \hat{\alpha}_{1}, \ldots, \hat{\alpha}_{l}\right\}$. Let $\tau_{2}$ be an automorphism of $\mathfrak{A}$ stabilizing $\mathfrak{H}$ such that $\tau_{2}^{*}=w\left(\tau_{2}\right.$ exists by [8, p. 276]). Theorem 1(b) says that $\tau_{2} \in \tilde{\Gamma}$; Theorem $1(\mathrm{c})$ then says that $\tau_{2} \in \tilde{\Gamma}_{0}$. Thus $\tau_{2}^{*} \eta_{1}^{*}=\left(\eta_{1} \tau_{2}\right)^{*}=\left(\eta \tau_{1} \tau_{2}\right)^{*}$ stabilizes the simple system.

This Corollary allows us to identify the components of $\Gamma$ by identifying the components of $\tilde{\Gamma}$. We recall from the Introduction that if $\eta \in$ Aut $\mathfrak{\AA}$, then there exists $\tau \in$ Aut $_{0} \mathfrak{R}$ such that $\eta \tau$ stabilizes $\mathfrak{S}$ and $(\eta \tau)^{*}$ permutes the roots of the given simple system. The inverse of this permutation of the simple roots, which can be identified with an automorphism of the Dynkin diagram of $\Re$, gives the component of Aut $\Omega$ in which $\eta$ lies. We now see that if $\eta \in \tilde{\Gamma}$ then we can choose $\tau \in \tilde{\Gamma}_{0}$. Thus the resulting graph automorphism must permute the roots $\rho_{1}, \ldots, \rho_{r}$ and also must permute the roots $\hat{\alpha}_{1}, \ldots, \hat{\alpha}_{l}$. Conversely, if $\eta$ is an automorphism of $\Re$ such that $\eta$ stabilizes $\mathfrak{S}$ and $\eta^{*}$ permutes $\rho_{1}, \ldots, \rho_{r}$ and also $\hat{\alpha}_{1}, \ldots, \hat{\alpha}_{l}$, then $\eta^{*}$ permutes the 
roots $\rho$ such that $\rho\left(R_{1}\right)=1$ (all such $\rho$ have the form $\rho_{i}+\hat{\alpha}, \alpha \in \mathfrak{S}_{1}^{*}$, by Proposition 8), and hence $\eta \in \tilde{\Gamma}$.

We conclude that the finite group $\Gamma / \Gamma_{0}$ is naturally isomorphic to the group of automorphisms of the Dynkin diagram of $\mathfrak{\Re}$ which stabilize $\rho_{1}, \ldots, \rho_{r}$. Here the vertices of the diagram are the simple roots $\rho_{1}, \ldots, \rho_{r}, \hat{\alpha}_{1}, \ldots, \hat{\alpha}_{l}$.

We now try to analyse $G$ in a manner analogous to the analysis of $\Gamma$ given above. We note first that if $\sigma$ is a root of $\Re$ such that $\sigma \varepsilon^{*}=\sigma$ and if $L$ is a root vector for $\sigma$, then $L \varepsilon= \pm L$. For $\mathfrak{\Omega}_{\sigma} \varepsilon=\mathfrak{\Omega}_{\sigma}$, i.e. $L \varepsilon=\lambda L$ for some $\lambda \in \Phi$; and the eigenvalues of $\varepsilon$ are \pm 1 . Since $\varepsilon$ interchanges $\Im$ and $\bar{\Im}$ and $L \in \Im$, $\Im$, or $\mathfrak{R}^{\prime}$, we must have $L \in \mathfrak{R}^{\prime}$. If $L \varepsilon=L$ then $L \in \mathfrak{D}$, whereas if $L \varepsilon=-L$ then $L \in R_{\mathfrak{J}^{\prime}}$. Conversely, if $L$ is in $R_{\mathfrak{I}}$ or $\mathfrak{D}$ and $L$ is a root vector for a root $\sigma$ of $\Re$, then $L \varepsilon= \pm L$ and hence $\sigma \varepsilon^{*}=\sigma$.

Proposition 21. Let $\chi$ be an element of $\tilde{\Gamma}$ which stabilizes $\mathfrak{S}$ and such that $\chi^{*}$ commutes with $\varepsilon^{*}$ (in their action on $\mathfrak{S}^{*}$ ). Let $R_{a}$ be a root vector for a root $\sigma$ of $\mathfrak{A}$. Then $R_{a} \chi=R_{b}$ for some $b \in \mathfrak{~}$.

Proof. In any event, $R_{a} \chi$ is a root vector for the root $\sigma \chi^{*-1}$. And $\sigma \chi^{*-1} \varepsilon^{*}$ $=\sigma \varepsilon^{*} \chi^{*-1}=\sigma \chi^{*-1}$. Thus a root vector for $\sigma \chi^{*-1}$ must belong either to $R_{\mathfrak{I}}$ or to $\mathfrak{D}$. We prove that it belongs to $R_{\mathfrak{J}}$.

In any event $\chi$ stabilizes $\mathfrak{W}$. For $\chi^{*}$ commutes with $\varepsilon^{*}$, so that $\chi \mid \mathfrak{S}$ and $\varepsilon \mid \mathfrak{F}$ commute. If $D \in \mathfrak{W}$, then $D_{\chi \varepsilon}=D_{\varepsilon \chi}=D_{\chi}$, which means that $D_{\chi} \in \mathfrak{W}$. This in turn implies that $\chi^{*}$ stabilizes $P$ (here $P$ is as in the discussion following Proposition 12); we know, of course, that $\chi^{*}$ permutes the roots $\rho$ such that $\rho\left(R_{1}\right)=1$; and if $\rho(\mathfrak{Z G})$ $=0$, then $\left(\rho \chi^{*}\right)(\mathfrak{W})=\rho(\mathfrak{W} \chi)=\rho(\mathfrak{W})=0$.

Let $\eta \in \Gamma$ be such that $\chi=\tilde{\eta}$. Let $1=\sum_{\rho \in P} e_{\rho}$ as in Proposition 13. If $\rho \in P$ then $e_{\rho} \eta^{-1}=e_{\rho} \chi^{-1} \in \mathfrak{R}_{\rho \chi^{*}} \subseteq \mathfrak{A}$. This means that $1 \eta^{-1}=\sum_{\rho \in P} e_{\rho} \eta^{-1} \in \mathfrak{A}$. Hence $R_{1 \eta}{ }^{-1} \in R_{\mathfrak{U}} \subseteq \mathfrak{F}_{\mathrm{g}}$. But if $x \in \mathfrak{A}$, then $\left[R_{a}, R_{x}\right]=\sigma\left(R_{x}\right) R_{a}=\left(\sigma \varepsilon^{*}\right)\left(R_{x}\right) R_{a}=\sigma\left(R_{x} \varepsilon\right) R_{a}$ $=\sigma\left(-R_{x}\right) R_{a}=-\sigma\left(R_{x}\right) R_{a}=-\left[R_{a}, R_{x}\right]$, from which we conclude that $\left[R_{a}, R_{1 \eta^{-1}}\right]=0$.

Now suppose $R_{a} \chi$ is a derivation. Then $0=(1)\left(R_{a} \chi\right)=1 \eta^{-1} R_{a} \eta=\left(1 \eta^{-1} \cdot a\right) \eta$ and, since $\eta$ is nonsingular, $1 \eta^{-1} \cdot a=0$. Also $a .\left(1 \eta^{-1}\right)^{.2}=\left(1 \eta^{-1}\right) R_{1 \eta^{-1}} R_{a}=\left(1 \eta^{-1}\right) R_{a} R_{1 \eta^{-1}}$ $=\left(1 \eta^{-1} \cdot a\right) R_{1 \eta^{-1}}=0$. Thus $a U_{1 \eta}-1=2\left(a .1 \eta^{-1}\right) \cdot 1 \eta^{-1}-a \cdot\left(1 \eta^{-1}\right)^{.2}=0$. This means that $a=0$ (since $1 \eta^{-1}$ is invertible)-a contradiction.

Proposition 22. Suppose $\eta \in \Gamma$ is such that $\tilde{\eta}$ commutes with $\varepsilon$. Then there exists $\zeta \in G$ such that for every $L \in \mathfrak{R}, \zeta^{-1} L \zeta=\eta^{-1} L \eta$.

Proof. Since $\tilde{\eta}$ commutes with $\varepsilon$, it stabilizes $R_{\mathfrak{J}}$. Indeed, if $L \in R_{\mathfrak{J}}$, then $L \tilde{\eta} \varepsilon$ $=L \varepsilon \tilde{\eta}=-L \tilde{\eta}$ and so $L \tilde{\eta} \in R_{\mathfrak{I}}$ (similarly, $\tilde{\eta}$ stabilizes $\mathfrak{D}$ ). Let $\zeta$ be the unique linear transformation of $\Im$ satisfying $R_{a \zeta}=R_{a} \tilde{\eta}$ for all $a \in \mathfrak{\Im}$. It is clearly sufficient to prove that $\zeta$ is an automorphism of $\Im$ : for then $R_{a \zeta}=\zeta^{-1} R_{a} \zeta$.

Let $x \in \mathfrak{\Im}$. Then $(x \eta)^{-}=x \eta \varepsilon=x \tilde{\eta} \varepsilon=x \varepsilon \tilde{\eta}=\bar{x} \tilde{\eta}=\left(x \eta^{\#-1}\right)^{-}$. Hence $\eta=\eta^{\#-1}$. Let $a$, $b \in \mathfrak{~}$. Then $2\left(R_{a \eta . b \eta}-\left[R_{a \eta}, R_{b \eta}\right]\right)=\left[a \eta,(b \eta)^{-}\right]=\left[a \eta,\left(b \eta^{\#-1}\right)^{-}\right]=[a \tilde{\eta}, \bar{b} \tilde{\eta}]=[a, \bar{b}] \tilde{\eta}=$ $2\left(R_{a . b} \tilde{\eta}-\left[R_{a}, R_{b}\right] \tilde{\eta}\right)$. Equating components in $R_{\mathfrak{J}}$ and $\mathfrak{D}$, we find that

(i) $R_{a . b} \tilde{\eta}=R_{a \eta . b \eta}$

(ii) $\left[R_{a}, R_{b}\right] \tilde{\eta}=\left[R_{a \eta}, R_{b \eta}\right]$. 
Taking $b=1$ in (ii) we see that $0=\left[R_{a \eta}, R_{1 \eta}\right]$, i.e. since $\eta$ is surjective, $\left[R_{\mathfrak{J}}, R_{1 \eta}\right]=0$. In other words, for all $x, y \in \Im$ the associator $[x, y, 1 \eta]$ is zero. Taking $a=b=1$ in (i), we get $R_{1}=R_{1} \tilde{\eta}=R_{1 \eta .1 \eta}$, i.e. $(1 \eta)^{2}=1$. In general, (i) is equivalent to $(a . b) \zeta$ $=a \eta \cdot b \eta$. Finally,

$$
\begin{aligned}
(a . b) \zeta & =(a . b) \zeta .(1 \eta)^{2}=(a \eta \cdot b \eta) \cdot(1 \eta \cdot 1 \eta) \\
& =[(a \eta . b \eta) .1 \eta] .1 \eta=[a \eta \cdot(b \eta .1 \eta)] .1 \eta \\
& =(a \eta . b \zeta) .1 \eta=(b \zeta . a \eta) .1 \eta=b \zeta .(a \eta .1 \eta) \\
& =b \zeta . a \zeta=a \zeta . b \zeta .
\end{aligned}
$$

$\zeta$ is nonsingular since $\tilde{\eta}$ is. Thus $\zeta$ is an automorphism.

We now prove an analogue to Theorem 1, (a) and (b).

THEOREM 2. (a) If $\eta \in G$, then there exists $\tau \in G_{0}$ so that $\tilde{\eta} \tilde{\tau}$ stabilizes $\mathfrak{H}$.

(b) If $\zeta \in G$ is such that $\tilde{\zeta}$ stabilizes $\mathfrak{H}_{2}$, then $\tilde{\zeta}^{*}$ (acting on $\mathfrak{S}^{*}$ ) permutes the roots of $\mathfrak{R}$, permutes the roots $\rho$ such that $\rho\left(R_{1}\right)=1$, and commutes with $\varepsilon^{*}$. Conversely, if $w$ is a linear transformation of $\mathfrak{S}^{*}$ which permutes the roots of $\AA$, permutes the roots $\rho$ such that $\rho\left(R_{1}\right)=1$, and commutes with $\varepsilon^{*}$, then there exists a $\zeta \in G$ such that $\tilde{\zeta}$ stabilizes $\mathfrak{S}$ and $\tilde{\zeta}^{*}=w$.

Proof. (a) $\mathfrak{W}_{1} \check{\eta}$ is a Cartan subalgebra of $\mathfrak{D}^{\prime}$. Thus there is an element of $\mathrm{Aut}_{0} \mathfrak{D}^{\prime}$ which maps $\mathfrak{W}_{1} \check{\eta}$ to $\mathfrak{W}_{1}$. By Proposition 5 , we can choose $\tau \in G_{0}$ so that $\mathfrak{W}_{1} \check{\eta} \check{\tau}=\mathfrak{W}_{1}$. Certainly $\mathfrak{E} \tilde{\eta} \tilde{\tau}=\mathfrak{E}$, which means that $\mathfrak{W} \tilde{\eta} \tilde{\tau}=\mathfrak{W}$. This implies that $\mathfrak{S} \tilde{\eta} \tilde{\tau}$ is a Cartan subalgebra of $\mathfrak{L}$ containing $\mathfrak{W}$. We conclude from Proposition 1 that $\mathfrak{S} \tilde{\eta} \tilde{\tau} \tilde{\tau}=\mathfrak{S}$.

(b) The first sentence is clear: $\tilde{\zeta}$ commutes with $\varepsilon$ on $\Re$-indeed,

$$
\begin{aligned}
\left(a+\bar{b}+R_{c}+D\right) \varepsilon \tilde{\zeta} & =\left(b+\bar{a}-R_{c}+D\right) \tilde{\zeta}=b \zeta+(a \zeta)^{-}-R_{c \zeta}+\zeta^{-1} D \zeta \\
& =\left(a \zeta+(b \zeta)^{-}+R_{c \zeta}+\zeta^{-1} D \zeta\right) \varepsilon \\
& =\left(a+\bar{b}+R_{c}+D\right) \tilde{\zeta} \varepsilon .
\end{aligned}
$$

In particular, $\tilde{\zeta}$ and $\varepsilon$ commute on $\mathfrak{H}$, which means that $\tilde{\zeta}^{*}$ and $\varepsilon^{*}$ commute.

We now prove the converse. We will find an $\eta \in \Gamma$ such that $\tilde{\eta}$ commutes with $\varepsilon$, stabilizes $\mathfrak{S}$, and satisfies $\tilde{\eta}^{*}=w$. This will be sufficient, in view of Proposition 22 . For if $\zeta$ is the automorphism given there, then $\tilde{\zeta}$ and $\tilde{\eta}$ agree on $\mathfrak{H} \subseteq \mathfrak{Q}$, and hence $\tilde{\zeta}^{*}=\tilde{\eta}^{*}$.

Choose an automorphism $\varphi$ of $\mathfrak{R}$ which stabilizes $\mathfrak{S}$ and such that $\varphi^{*}=w(\varphi$ exists by results in Chapter 9 of [8]). Let $\lambda=\varepsilon^{-1} \varphi^{-1} \varepsilon \varphi=\varepsilon \varphi^{-1} \varepsilon \varphi$. Now since $\varphi^{*}$ and $\varepsilon^{*}$ commute, $\varphi \mid \mathfrak{S}$ and $\varepsilon \mid \mathfrak{H}$ commute. This means that $\lambda$ fixes $\mathfrak{S}$ pointwise and hence that on each root space $\Re_{\rho}$ of $\Re, \lambda$ is multiplication by some scalar $\lambda_{\rho} \in \Phi$. Let $\rho$ be a root of $\mathfrak{\AA}$ and $0 \neq K \in \mathfrak{\Re}_{\rho}$. Then $K \varepsilon \in \mathfrak{\Re}_{\rho \varepsilon^{*}}$ and $K \varepsilon \lambda=K \varepsilon \varepsilon \varphi^{-1} \varepsilon \varphi=$ $K \varphi^{-1} \varepsilon \varphi \varepsilon \varepsilon=K \lambda^{-1} \varepsilon=\lambda_{\rho}^{-1} K \varepsilon$. Thus $\lambda_{\rho \varepsilon^{*}}=\lambda_{\rho}^{-1}$. Suppose $\rho$ is fixed by $\varepsilon^{*}$. We claim that $\lambda_{\rho}=1$. There are two cases to consider.

Case 1. $\rho$ has a root vector of the form $R_{a}, a \in \Im$. Then $R_{a} \lambda=R_{a} \varepsilon \varphi^{-1} \varepsilon \varphi$ $=-R_{a} \varphi^{-1} \varepsilon \varphi$. Theorem 1(b) says that $\varphi \in \tilde{\Gamma}$, and Proposition 21 then shows that $R_{a} \varphi^{-1} \in R_{\mathfrak{J}}$. Thus $R_{a} \lambda=-\left(-R_{a} \varphi^{-1}\right) \varphi=R_{a}$. 
Case 2. $\rho$ has a root vector of the form $D \in \mathscr{D}$. Then $D \varphi^{-1} \in \mathfrak{D}$. Indeed, since $\rho \varphi^{*}$ is fixed by $\varepsilon^{*}$, the only other possibility is $D \varphi^{-1}=R_{a}$ for some $a \in \Im$, i.e. $R_{a} \varphi$ $=D$, which contradicts Proposition 21. But then $D \lambda=D \varepsilon \varphi^{-1} \varepsilon \varphi=\left(D \varphi^{-1}\right) \varepsilon \varphi$ $=D \varphi^{-1} \varphi=D$.

Now let $\alpha_{1}, \ldots, \alpha_{l}$ be a simple system of roots of $\mathfrak{Q}^{\prime}$ stabilized by $\varepsilon^{*}$ (Proposition 15), and let $\rho_{1}, \ldots, \rho_{r}, \hat{\alpha}_{1}, \ldots, \hat{\alpha}_{l}$ be the corresponding simple system of roots of $\mathfrak{R}$ as in Proposition 9. As has been observed at the beginning of this section, for any nonzero $\theta_{\rho_{1}}, \ldots, \theta_{\alpha_{l}} \in \Phi$ (one scalar for each simple root) there is a unique automorphism $\theta$ of $\mathfrak{R}$ which fixes $\mathfrak{S}$ pointwise and on the root space $\mathfrak{R}_{\rho}$ of each simple root $\rho$ is scalar multiplication by $\theta_{\rho}$. We choose a set of scalars as follows:

(i) If $\hat{\alpha}_{i} \varepsilon^{*}=\hat{\alpha}_{i}$, let $\theta_{\hat{\alpha}_{i}}=1$.

(ii) If $\varepsilon^{*}$ interchanges $\hat{\alpha}_{i}$ and $\hat{\alpha}_{j}, i<j$, take $\theta_{\hat{\alpha}_{l}}=1$ and $\theta_{\alpha_{j}}=\lambda_{\alpha_{i}}$.

(iii) For each $\rho_{i}$ there exist nonnegative integers $n_{1}, \ldots, n_{l}$ (depending on $\rho_{i}$ ) such that $\rho_{i} \varepsilon^{*}=-\rho_{i}-\sum_{t=1}^{l} n_{t} \hat{\alpha}_{t}$. Here we use Proposition 14. Take $\theta_{\rho_{i}}$ to be a square root of $\left(\lambda_{\rho_{i}} \theta_{\hat{\alpha}_{1}}^{n_{1}} \ldots \theta_{\hat{\alpha}_{l}}^{n_{l}}\right)^{-1}$. Note that $\theta_{\hat{\alpha}_{1}}, \ldots, \theta_{\hat{\alpha}_{l}}$ have already been chosen.

Recall that on the root space of the root $\rho=\sum k_{i} \rho_{i}+\sum m_{i} \hat{\alpha}_{i}, \theta$ is scalar multiplication by $\theta_{\rho_{1}}^{k_{1}} \cdots \theta_{\alpha_{l}}^{m_{l}} \equiv \theta_{\rho}$. Similarly, $\lambda_{\rho}=\lambda_{\rho_{1}}^{k_{1}} \cdots \lambda_{\alpha_{l} l}^{m_{l}}$.

We claim that, for all roots $\rho$ of $\Re, \lambda_{\rho} \theta_{\rho}=\theta_{\rho \varepsilon^{*}}$. We first show this for $\rho$ a simple root. If $\hat{\alpha}_{i} \varepsilon^{*}=\hat{\alpha}_{i}$, then $\lambda_{\hat{\alpha}_{i}}=1=\theta_{\hat{\alpha}_{i}}=\theta_{\hat{\alpha}_{i} \varepsilon^{*}}$, and the result is clear. If $\varepsilon^{*}$ interchanges $\hat{\alpha}_{i}$ and $\hat{\alpha}_{j}, i<j$, then $\lambda_{\alpha_{i}} \theta_{\alpha_{i}}=\lambda_{\alpha_{i}} \cdot 1=\lambda_{\alpha_{i}}=\theta_{\alpha_{j}}$ and $\lambda_{\alpha_{j}} \theta_{\alpha_{j}}=\lambda_{\alpha_{i}}{ }^{-1} \lambda_{\alpha_{i}}=1=\theta_{\alpha_{i}}$. Finally, if $\rho_{i} \varepsilon^{*}=-\rho_{i}-\sum_{t=1}^{l} n_{t} \hat{\alpha}_{t}$ then

$$
\theta_{\rho_{i} \varepsilon^{*}}=\theta_{\rho_{i}}^{-1} \theta_{\hat{\alpha}_{1}}^{-n_{1}} \ldots \theta_{\alpha_{l}}^{-n_{l}}=\theta_{\rho_{i}} \theta_{\rho_{l}}^{-2} \theta_{\alpha_{1}}^{-n_{1}} \ldots \theta_{\dot{\alpha}_{l}}^{-n_{l}}=\theta_{\rho_{l}} \lambda_{\rho_{i}}
$$

by the way $\theta_{\rho_{i}}$ was chosen. Now let $\rho=\sum k_{i} \rho_{i}+\sum m_{i} \hat{\alpha}_{i}$ be any root. Then

$$
\begin{aligned}
\theta_{\rho} \lambda_{\rho} & =\prod_{i} \theta_{\rho_{i}}^{k_{i}} \prod_{i} \theta_{\hat{\alpha}_{i}}^{m_{i}} \prod_{i} \lambda_{\rho_{i}}^{k_{i}} \prod_{i} \lambda_{\alpha_{i}}^{m_{i}} \\
& =\prod_{i}\left(\theta_{\rho_{i}} \lambda_{\rho_{i}}\right)^{k_{i}} \prod_{i}\left(\theta_{\alpha_{i}} \lambda_{\alpha_{i}}\right)^{m_{i}} \\
& =\prod_{i} \theta_{\rho_{i} \varepsilon^{*}}^{k_{i}} \prod_{i} \theta_{\alpha_{i} \varepsilon^{*}}^{m_{i}}=\theta_{\rho \varepsilon^{*}}
\end{aligned}
$$

This last equality holds because $\rho_{1} \varepsilon^{*}, \ldots, \rho_{r} \varepsilon^{*}, \hat{\alpha}_{1} \varepsilon^{*}, \ldots, \hat{\alpha}_{l} \varepsilon^{*}$ is also a simple system of roots and $\rho \varepsilon^{*}=\sum k_{i} \rho_{i} \varepsilon^{*}+\sum m_{i} \hat{\alpha}_{i} \varepsilon^{*}$ (i.e. $\theta$ is determined by its action on the root spaces of the roots in this simple system).

We now conclude the proof. $\theta \in \tilde{\Gamma}$ since it fixes $R_{1} \in \mathfrak{H}$. Also $\varphi \in \tilde{\Gamma}$ by Theorem 1(b). Let $\eta=\varphi \theta \in \tilde{\Gamma}$. On $\mathfrak{S} \eta$ agrees with $\varphi$. Hence $\eta^{*}=\varphi^{*}=w$. Also $\varphi$ commutes with $\varepsilon$ on $\mathfrak{H}$, so $\eta$ does. Let $K$ be a root vector for a root $\rho$ of $\mathfrak{R}$. Then $K \varepsilon \eta^{-1} \varepsilon \eta$ $=(K \varepsilon) \theta^{-1} \varphi^{-1} \varepsilon \varphi \theta=\theta_{\rho \varepsilon^{*}}^{-1} K \varepsilon \varphi^{-1} \varepsilon \varphi \theta$ (since $K \varepsilon$ is a root vector for $\left.\rho \varepsilon^{*}\right)=\theta_{\rho \varepsilon^{*}}^{-1} \lambda_{\rho} K \theta$ $=\theta_{\rho \varepsilon}^{-1} \cdot \lambda_{\rho} \theta_{\rho} K=K$. Thus $\eta$ commutes with $\varepsilon$ on $\Re_{\rho}$. Hence $\eta$ commutes with $\varepsilon$ everywhere on $\mathfrak{R}$, as desired.

Henceforth $\left\{\alpha_{1}, \ldots, \alpha_{l}\right\}$ is a fixed simple system of roots of $\mathfrak{L}^{\prime}$ stabilized by $\varepsilon^{*}$, and $\rho_{1}, \ldots, \rho_{r}$ are as given in Proposition 9. 
PROPOSITION 23. Let $w$ be a linear transformation of $\mathfrak{S}^{*}$ which permutes the roots of $\Re$, permutes the roots $\rho_{1}, \ldots, \rho_{r}$ and also permutes the roots $\hat{\alpha}_{1}, \ldots, \hat{\alpha}_{l}$. Then $w$ commutes with $\varepsilon^{*}$.

Proof. Let $\eta \in \Gamma$ be such that $\tilde{\eta}$ stabilizes $\mathfrak{H}$ and $\tilde{\eta}^{*}=w$ (here we are using Theorem 1(b)). Choose $x \in \Im$ such that $x$ is invertible and $x^{2}=1 \eta$. This is possible since $1 \eta$ is invertible and the field is algebraically closed [10, p. 242]. Then $1 \eta U_{x}^{-1}$ $=x^{2} U_{x}^{-1}=1$, and hence $\eta_{1}=\eta U_{x}^{-1}$ is an automorphism of $\Im$. Also $U_{x}$ is in $\Gamma_{0}$ (the set of invertible elements of $\mathfrak{\Im}$ is a nonempty Zariski open subset of $\mathfrak{\Im}$; hence it is irreducible, so that its image under the polynomial map $x \mapsto U_{x}$ is also irreducible). Choose an automorphism $\tau \in G_{0}$ so that $\tilde{\eta}_{1} \tilde{\tau}$ stabilizes $\mathfrak{H}$ (here we appeal to Theorem 2(a)). Thus $\eta_{2}=\eta U_{x}^{-1} \tau$ is an automorphism of $\mathfrak{\Im}$ such that $\tilde{\eta}_{2}$ stabilizes $\mathfrak{F}$. Now $\eta_{2}^{-1} \eta=\tau^{-1} U_{x} \equiv \zeta$ is in $\Gamma_{0}$ and $\tilde{\zeta}$ stabilizes $\mathfrak{S}$. So by Theorem $1(\mathrm{c}),\left(\tilde{\eta}_{2}^{-1} \tilde{\eta}\right)^{*}=\tilde{\eta}^{*} \tilde{\eta}_{2}^{*-1}$ $=\tilde{\zeta}^{*}$ is in the Weyl group of $\Re$. Note that since $\eta_{2}$ is an automorphism of $\Im, \tilde{\eta}_{2}^{*-1}$ commutes with $\varepsilon^{*}$. For brevity let $\tilde{\eta}_{2}^{*-1}=v$. Then we have $w v=\tilde{\zeta}^{*}$, where $w$ stabilizes $\left\{\rho_{1}, \ldots, \rho_{r}\right\}$ and $\left\{\hat{\alpha}_{1}, \ldots, \hat{\alpha}_{l}\right\}, v$ permutes the roots $\rho$ such that $\rho\left(R_{1}\right)=1$ and com-

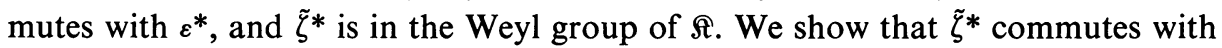
$\varepsilon^{*}$. Indeed, $\varepsilon^{*} \tilde{\zeta} \varepsilon^{*}$ is in the Weyl group, since if $w_{\rho}$ is the symmetry of $\mathfrak{S}^{*}$ in the direction of the root $\rho$, then $\varepsilon^{*} w_{\rho} \varepsilon^{*}=w_{\rho \varepsilon^{*}}$. Since $w$ and $\varepsilon^{*}$ stabilize $\left\{\hat{\alpha}_{1}, \ldots, \hat{\alpha}_{l}\right\}$, we have $\left\{\hat{\alpha}_{1}, \ldots, \hat{\alpha}_{l}\right\} \varepsilon^{*} \tilde{\zeta}^{*} \varepsilon^{*} \tilde{\zeta}^{*-1}=\left\{\hat{\alpha}_{1}, \ldots, \hat{\alpha}_{l}\right\} \varepsilon^{*} w v \varepsilon^{*} v^{-1} w^{-1}=\left\{\hat{\alpha}_{1}, \ldots, \hat{\alpha}_{l}\right\} \varepsilon^{*} w \varepsilon^{*} v v^{-1} w^{-1}$ $=\left\{\hat{\alpha}_{1}, \ldots, \hat{\alpha}_{l}\right\} \varepsilon^{*} w \varepsilon^{*} w^{-1}=\left\{\hat{\alpha}_{1}, \ldots, \hat{\alpha}_{l}\right\}$. I.e. $\varepsilon^{*} \tilde{\zeta}^{*} \varepsilon^{*} \tilde{\zeta}^{*-1}$ is an element of the Weyl group of $\AA$ which permutes the roots $\rho$ such that $\rho\left(R_{1}\right)=1$ and stabilizes $\left\{\hat{\alpha}_{1}, \ldots, \hat{\alpha}_{l}\right\}$. By the Corollary to Proposition 9 it also stabilizes $\left\{\rho_{1}, \ldots, \rho_{r}\right\}$. An element of the Weyl group which stabilizes a simple system of roots is equal to 1 . I.e., $\tilde{\zeta}^{*}=\varepsilon^{*} \tilde{\zeta}^{*} \varepsilon^{*}$. Thus $w=\tilde{\zeta}^{*} v^{-1}$ commutes with $\varepsilon^{*}$.

If $u$ is a linear transformation of $\mathfrak{W}_{1}^{*}$, we let $\bar{u}$ be the linear transformation of $\mathfrak{2} \mathfrak{F}^{*}$ which fixes the points of $\mathfrak{C}^{*}$ and which for $\omega \in \mathfrak{W}_{1}^{*}$ satisfies $\bar{\omega} \bar{u}=(\omega u)^{-}$.

Let $w$ be a linear transformation of $\mathfrak{S}^{*}$ which permutes the roots of $\mathfrak{R}$, permutes the roots $\rho$ such that $\rho\left(R_{1}\right)=1$, and commutes with $\varepsilon^{*}$. Then $w$ stabilizes $\left(\mathfrak{S}_{1}^{*}\right)^{\wedge}$ and $w \mid\left(\mathfrak{S}_{1}^{*}\right)^{\wedge}=\hat{v}$ for some $v \in \operatorname{Hom}_{\Phi}\left(\mathfrak{S}_{1}^{*}, \mathfrak{S}_{1}^{*}\right)$ which permutes the roots of $\mathfrak{R}^{\prime}$ and commutes with $\varepsilon^{*}$. So $v$ stabilizes $\mathfrak{U}^{*}$ and $\mathfrak{W}^{*}$, and $v \mid \mathfrak{W}^{*}$ permutes the roots of $\Sigma_{\varepsilon}$. Theorem 2(b) implies that there is a $\zeta \in G$ such that $\tilde{\zeta}$ stabilizes $\mathfrak{H}$ and $\tilde{\zeta}^{*}=w$. Now $\tilde{\zeta}$ also stabilizes $\mathfrak{S}_{1}=\mathfrak{S} \cap \mathfrak{R}^{\prime}$; we claim that $v=\tilde{\zeta}^{*}$ (acting on $\mathfrak{S}_{1}^{*}$ ). In fact if $H \in \mathfrak{S}_{1}$ and $\alpha \in \mathfrak{S}_{1}^{*}$ then $\left(\alpha \tilde{\zeta}^{*}\right)(H)=\alpha(H \tilde{\zeta})=\hat{\alpha}(H \tilde{\zeta})=\hat{\alpha} \tilde{\zeta}^{*}(H)=\hat{\alpha} \hat{v}(H)=(\alpha v)^{\wedge}(H)$ $=\alpha v(H)$. Now since $\zeta$ is an automorphism of $\mathfrak{\Im}, \tilde{\zeta}$ stabilizes $R_{\mathfrak{J}}$ and $\mathfrak{D}$, hence also $R_{\mathfrak{U}^{\prime}}$, $\mathfrak{E}$, and $\mathfrak{W}_{1}$. This means that $\tilde{\zeta}^{*}$ stabilizes $\mathfrak{U}^{*}, \mathfrak{C}^{*}$, and $\left(\mathfrak{W}_{1}^{*}\right)^{-}$: for example, if $\alpha \in \mathfrak{F}^{*}$, then $\left(\alpha \tilde{\zeta}^{*}\right)\left(R_{\mathfrak{U}^{\prime}}+\mathfrak{W}_{1}\right)=\alpha\left(\left(R_{\mathfrak{U}^{\prime}}+\mathfrak{W}_{1}\right) \tilde{\zeta}\right)=\alpha\left(R_{\mathfrak{U}^{\prime}}+\mathfrak{W}_{1}\right)=0$, so that $\alpha \tilde{\zeta}^{*} \in \mathfrak{F}^{*}$. We conclude that $v$ stabilizes $\mathfrak{A}^{*}$, $\mathfrak{F}^{*}$, and $\left(\mathfrak{W}_{1}^{*}\right)^{-}$.

Suppose now that $\omega$ is a root of $\mathfrak{D}^{\prime}$; choose a root vector $R_{a}+B, B \neq 0$, for a root $\beta$ of $\mathfrak{L}^{\prime}$ such that $\beta \mid \mathfrak{W}_{1}=\omega$ (here we are using Proposition $19(\mathrm{a})$ ). Now $\beta(\mathfrak{F})=0$ by the Corollary to Proposition 16. Hence $\beta_{+}=\bar{\omega}$. Now $\beta v=\beta \tilde{\zeta}^{*}$ has a root vector $\left(R_{a}+B\right) \tilde{\zeta}^{-1}=\zeta\left(R_{a}+B\right) \zeta^{-1}=R_{a \zeta^{-1}}+\zeta B \zeta^{-1}$. Here $0 \neq \zeta B \zeta^{-1} \in \mathfrak{D}$. We use Proposition 
17 to conclude that $(\beta v) \mid \mathfrak{W}_{1}$ is a $\operatorname{root} \psi$ of $\mathfrak{D}^{\prime}$. Now $\bar{\psi}=(\beta v)_{+}=\left(\left(\beta_{+}+\beta_{-}\right) v\right)_{+}$ $=\beta_{+} v=\omega v$. We see that we have proven the following:

Proposition 24. Let $w$ be a linear transformation of $\mathfrak{S}^{*}$ which permutes the roots of $\Re$, permutes the roots $\rho$ such that $\rho\left(R_{1}\right)=1$, and commutes with $\varepsilon^{*}$. Suppose $v$ is the linear transformation of $\mathfrak{S}_{1}^{*}$ such that $\hat{v}=w \mid\left(\mathfrak{S}_{1}^{*}\right)^{\wedge}$. Then $v$ stabilizes $\mathfrak{U}^{*}$, $\mathfrak{F}^{*}$, and $\left(\mathfrak{I B}_{1}^{*}\right)^{-}$, and also $v$ permutes among themselves the elements $\bar{\omega}$ of $\left(\mathfrak{W B}_{1}^{*}\right)^{-}$, where $\omega$ is a root of $\mathfrak{D}^{\prime}$.

Proposition 25. (a) If $u$ is in the Weyl group of $\Sigma_{\varepsilon}$, then $u$ stabilizes f** and $\left(\mathfrak{B}_{1}^{*}\right)^{-}$and permutes among themselves the roots of $\Sigma_{\varepsilon}$ of the form $\bar{\omega}, \omega$ a root of $\mathfrak{D}^{\prime}$.

(b) If $u$ is in the Weyl group of $\mathfrak{D}^{\prime}$, then $\bar{u}$ is in the Weyl group of $\Sigma_{\varepsilon}$.

Proof. (a) By the theorem of Steinberg cited following Proposition 15, there is a unique element $v$ of the Weyl group of $\mathfrak{Q}^{\prime}$ which commutes with $\varepsilon^{*}$ and satisfies $u=v \mid \mathfrak{B} *$. By Proposition 11, there is a unique element $w$ of the Weyl group of $\mathfrak{\Omega}$ which permutes the roots $\rho$ such that $\rho\left(R_{1}\right)=1$, and which satisfies $w \mid\left(\mathfrak{H}_{1}^{*}\right)^{\wedge}=\hat{v}$. By Proposition 12,w commutes with $\varepsilon^{*}$. Hence Proposition 24 applies to $w$ and $v$, and the desired conclusion follows.

(b) It is clear that we need only prove this for $u$ the symmetry $w_{\omega}$ of $\mathfrak{W}_{1}^{*}$ in the direction of a root $\omega$ of $\mathfrak{D}^{\prime}$-for these symmetries generate the Weyl group of $\mathfrak{D}^{\prime}$. We show in fact that $\left(w_{\omega}\right)^{-}=w_{\bar{\omega}}$. These two linear transformations of $\mathfrak{W}^{*}$ agree on (5*; in fact they both fix it pointwise, the first by definition and the second because $\left\langle\bar{\omega}, \mathfrak{F}^{*}\right\rangle=0$. We show they agree on $\left(\mathfrak{W}_{1}^{*}\right)^{-}$. Let $\{$,$\} denote the Killing form on$ $\mathfrak{D}^{\prime}$ and the associated bilinear form on $\mathfrak{B}_{1}^{*}$. If $\psi \in \mathfrak{W}_{1}^{*}$ then $\bar{\psi} \bar{w}_{\omega}=\left(\psi w_{\omega}\right)^{-}=$ $\bar{\psi}-(2\{\psi, \omega\} /\{\omega, \omega\}) \bar{\omega}$, whereas $\bar{\psi} w_{\bar{\omega}}=\bar{\psi}-(2\langle\bar{\psi}, \bar{\omega}\rangle /\langle\bar{\omega}, \bar{\omega}\rangle) \bar{\omega}$. Part (a) of this proposition shows that $w_{\bar{\omega}}$ permutes the roots $\bar{\sigma}$ of $\Sigma_{\varepsilon}\left(\sigma\right.$ a root of $\left.\mathfrak{D}^{\prime}\right)$. Thus $\left(w_{\omega}\right)^{-} \mid\left(\mathfrak{N B}_{10}^{*}\right)^{-}$ and $w_{\bar{\omega}} \mid\left(\mathfrak{W}_{10}^{*}\right)^{-}$are two symmetries in the direction of the same vector $\bar{\omega}$ which stabilize a finite set of vectors spanning $\left(\mathfrak{I S}_{10}^{*}\right)^{-}$. By a lemma of Serre [13, V-2, Lemme 1], we conclude that $\left(w_{\omega}\right)^{-}=w_{\bar{\omega}}$.

Since the mapping $u \mapsto \bar{u}$ of $\operatorname{Hom}_{\Phi}\left(\mathfrak{W}_{1}^{*}, \mathfrak{W}_{1}^{*}\right)$ to $\operatorname{Hom}_{\Phi}\left(\mathfrak{W}^{*}, \mathfrak{W}^{*}\right)$ is injective and an algebra homomorphism, the above proposition in effect embeds the Weyl group of $\mathfrak{D}^{\prime}$ in the Weyl group of $\Sigma_{\varepsilon}$.

We continue with $w, v$, and $\zeta$ as in the two paragraphs preceding the statement of Proposition 24. Let $u$ be the linear transformation of $\mathfrak{W}_{1}^{*}$ such that $\bar{u} \mid\left(\mathfrak{W}_{1}^{*}\right)^{-}$ $=v \mid\left(\mathfrak{B}_{1}^{*}\right)^{-}$. Proposition 24 says that $u$ permutes the roots of $\mathfrak{D}^{\prime}$. Now $\breve{\zeta}$ stabilizes $\mathfrak{W}_{1}$; we claim that $u=\check{\zeta}^{*}$ (acting on $\mathfrak{W}_{1}^{*}$ ). Indeed, let $\omega \in \mathfrak{W}_{1}^{*}$ and $x \in \mathfrak{X}^{\prime}, E \in \mathfrak{E}$, $D \in \mathfrak{W}_{1}$. Then $\left(\bar{\omega}\left(\check{\zeta}^{*}\right)^{-}\right)\left(R_{x}+E+D\right)=\left(\omega \check{\zeta}^{*}\right)^{-}\left(R_{x}+E+D\right)=\left(\omega \check{\zeta}^{*}\right)(D)=\omega(D \check{\zeta})=$ $\omega\left(\zeta^{-1} D \zeta\right)=\omega(D \tilde{\zeta})=\bar{\omega}\left(R_{x \zeta}+E \tilde{\zeta}+D \tilde{\zeta}\right)=\left(\bar{\omega} \tilde{\zeta}^{*}\right)\left(R_{x}+E+D\right)=(\bar{\omega} v)\left(R_{x}+E+D\right)$. Thus $\bar{\omega} v=\bar{\omega}\left(\check{\zeta}^{*}\right)^{-}$for all $\omega \in \mathfrak{B}_{1}^{*}$, and $\check{\zeta}^{*}=u$.

Recall that the projection onto $\mathfrak{S}^{*}$ of our fixed simple system $\alpha_{1}, \ldots, \alpha_{l}$ of roots of $\mathfrak{R}^{\prime}$ gives a simple system, and hence an ordering, of $\Sigma_{\varepsilon}$. In this ordering, positive roots of $\mathfrak{R}^{\prime}$ project onto positive roots of $\Sigma_{\varepsilon}$. We can define an ordering of the roots of $\mathfrak{D}^{\prime}$ by the following rule: if $\omega$ is a root of $\mathfrak{D}^{\prime}$ then $\omega>0$ if and only if $\bar{\omega}>0$. The 
simple system of roots of $\mathfrak{D}^{\prime}$ associated with this ordering is the unique simple system consisting entirely of roots $\omega$ such that $\bar{\omega}>0$. Let $w$ be a linear transformation of $\mathfrak{S}^{*}$ which permutes the roots $\rho_{1}, \ldots, \rho_{r}$ and the roots $\hat{\alpha}_{1}, \ldots, \hat{\alpha}_{l}$. Then $w$ commutes with $\varepsilon^{*}$ by Proposition 23, and permutes the roots $\rho$ such that $\rho\left(R_{1}\right)=1$. Proposition 24 then applies to $w$ : if $w \mid\left(\mathfrak{S}_{1}^{*}\right)^{\wedge}=\hat{v}$, then $v$ permutes the roots $\bar{\omega}, \omega$ a root of $\mathfrak{D}^{\prime}$. Since $v$ permutes $\alpha_{1}, \ldots, \alpha_{l}$, it permutes the positive roots of $\mathfrak{Q}^{\prime}$. This means that $v \mid \mathfrak{W}^{*}$ permutes the positive roots of $\Sigma_{\varepsilon}$, i.e. stabilizes the standard simple system of $\Sigma_{\varepsilon}$. Finally if $v\left|\left(\mathfrak{W}_{1}^{*}\right)^{-}=\bar{u}\right|\left(\mathfrak{W}_{1}^{*}\right)^{-}$for $u \in \operatorname{Hom}_{\Phi}\left(\mathfrak{W}_{1}^{*}, \mathfrak{W} \mathfrak{3}_{1}^{*}\right)$, then $u$ permutes the positive roots of $\mathfrak{D}^{\prime}$; that is, if $\bar{\omega}>0$, then $(\omega u)^{-}=\bar{\omega} \bar{u}=\bar{\omega} v>0$. We conclude that $\bar{u}$ stabilizes the standard simple system of roots of $\mathfrak{D}^{\prime}$.

Let $w, v, \zeta$ be as in the two paragraphs preceding the statement of Proposition 24. By the Corollary to Theorem 1 , there is a $\tau \in \tilde{\Gamma}_{0}$ such that $\tilde{\zeta} \tau$ stabilizes $\mathfrak{S}^{*}$ and $(\tilde{\zeta} \tau)^{*}$ stabilizes $\rho_{1}, \ldots, \rho_{r}, \hat{\alpha}_{1}, \ldots, \hat{\alpha}_{l}$. Now $\tilde{\zeta}$ stabilizes $\mathfrak{S}^{*}$ so $\tau$ also stabilizes $\mathfrak{S}^{*}$. Hence Theorem 1(c) implies that $\tau^{*}$ is in the Weyl group of $\Re$. Since $\tilde{\zeta}^{*}$ and $\tau^{*} \tilde{\zeta}^{*}$ commute with $\varepsilon^{*}$ (the latter by Proposition 23), $\tau^{*}$ commutes with $\varepsilon^{*}$. These remarks allow us to prove the following:

Proposition 26. Let $w$ be a linear transformation in $\mathfrak{S}^{*}$ which permutes the roots of $\AA$, permutes the roots $\rho$ such that $\rho\left(R_{1}\right)=1$, and commutes with $\varepsilon^{*}$. Then there are elements $v_{1}, v_{2}$ of the Weyl group of $\Re$ which commute with $\varepsilon^{*}$ and permute the roots $\rho$ of $\Re$ such that $\rho\left(R_{1}\right)=1$, and such that $v_{1} w=w v_{2}$ permutes the roots $\rho_{1}, \ldots, \rho_{r}$ and the roots $\hat{\alpha}_{1}, \ldots, \hat{\alpha}_{l}$.

Proof. Choose $v, \zeta, \tau$ so that $w, v, \zeta, \tau$ are as above. Let $v_{1}=\tau^{*}$ and $v_{2}$ $=\tilde{\zeta}^{*-1} \tau^{*} \tilde{\zeta}^{*}$. Now $v_{1}$ is in the Weyl group and, since $\tilde{\zeta}$ is an automorphism of $\mathfrak{R}$, $\tilde{\zeta}^{*}$ is an orthogonal transformation of $\mathfrak{S}^{*}$ permuting the roots of $\Re$. Hence $v_{2}$ is in the Weyl group. The rest of the proposition then follows.

We now prove the following analogue of Theorem 1(c).

THEOREM 3. Let $\eta \in G$. Necessary and sufficient conditions for $\eta$ to be in $G_{0}$ are that

(i) $\eta$ commutes with every $E \in \mathbb{E}$,

(ii) $\check{\eta} \in$ Aut $_{0} \mathfrak{D}^{\prime}$, and

(iii) $\tilde{\eta} \in \mathrm{Aut}_{0} \Re$.

Proof. The necessity of these conditions was shown in Proposition 5 and in the remarks preceding it. So suppose $\eta$ satisfies these conditions. By Proposition 5, there exists $\theta \in G_{0}$ so that $\check{\theta}=\check{\eta}$. The automorphism $\eta \theta^{-1}$ of $\Im$ satisfies (i), (ii), and (iii) since both $\eta$ and $\theta$ do, and in addition commutes with all elements of $\mathfrak{D}^{\prime}$. Since it commutes with all elements of $\&$ it in fact commutes with all derivations of $\Im$. Also it belongs to the same component of $G$ as $\eta$. Thus we may assume, replacing $\eta$ by $\eta \theta^{-1}$, that for every $D \in \mathcal{D}, \eta^{-1} D \eta=D$.

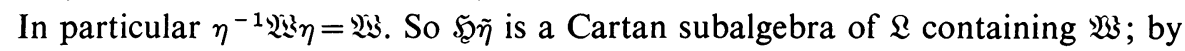
Proposition $1, \mathfrak{S} \tilde{\eta}=\mathfrak{S}$. Since $\tilde{\eta} \in \mathrm{Aut}_{0} \mathfrak{R}, \tilde{\eta}^{*}$ is in the Weyl group of $\Re$. Also $\tilde{\eta}$ permutes the roots $\rho$ such that $\rho\left(R_{1}\right)=1$; we apply Proposition 11 to conclude that $\tilde{\eta}^{*} \mid\left(\mathfrak{S}_{1}^{*}\right)^{\wedge}=\hat{v}$ where $v$ is in the Weyl group of $\mathfrak{L}^{\prime}$. 
Now $\tilde{\eta}^{*}$ commutes with $\varepsilon^{*}$ (since $\eta \in G$ ). Also $v=\tilde{\eta}^{*}$ (acting on $\mathfrak{S}_{1}^{*}$ ), as in the remarks preceding the statement of Proposition 24. So $v$ commutes with $\varepsilon^{*}$. We claim that $v$ fixes $\mathfrak{W}^{*}$ pointwise. Indeed, if $\omega \in \mathfrak{W}^{*}, x \in \mathfrak{H}^{\prime}$ and $D \in \mathfrak{W}$ then $(\omega v)\left(R_{x}+D\right)$ $=\omega\left(\left(R_{x}+D\right) \tilde{\eta}\right)=\omega\left(R_{v \eta}+D\right)=\omega(D)=\omega\left(R_{x}+D\right)$ and so $\omega v=\omega$. The theorem of Steinberg previously cited then implies that $v=1$. Proposition 11 in turn implies that $\tilde{\eta}^{*}=1$ : for 1 and $\tilde{\eta}^{*}$ are both extensions of $\hat{v}$ to an element of the Weyl group of $\mathfrak{K}$ permuting the roots $\rho$ such that $\rho\left(R_{1}\right)=1$. This means that if $\rho$ is any root of $\mathfrak{R}$ then $\mathfrak{N}_{\rho} \tilde{\eta}=\mathfrak{R}_{\rho}$, and that $\tilde{\eta}$ fixes $\mathfrak{S}$ pointwise. Our proof will then be complete if we prove the following:

LEMma. Let $\eta$ be an automorphism of $\Im$ which commutes with every derivation of $\Im$ and such that $\tilde{\eta}$ fixes a Cartan subalgebra of $\mathfrak{L}$ pointwise. Then $\eta \in G_{0}$.

Proof. We first show that it is sufficient to assume $\mathfrak{\Im}$ simple; to do this, we use induction on the number of simple summands of $\mathfrak{\Im}$. Indeed, suppose $\mathfrak{\Im}=\mathfrak{\Im}_{1} \oplus \mathfrak{\Im}_{2}$, where the $\mathfrak{I}_{i}$ are nonzero ideals of $\mathfrak{\Im}$. Then $\mathfrak{L}=\mathfrak{L}_{1} \oplus \mathfrak{L}_{2}$ and $\mathfrak{R}=\mathfrak{\Omega}_{1} \oplus \mathfrak{I}_{2}$; here $\mathfrak{L}_{i}=\mathfrak{L}\left(\mathfrak{\Im}_{i}\right)$ and $\mathfrak{\Re}_{i}=\mathfrak{\Re}\left(\mathfrak{\Im}_{i}\right)(i=1,2)$. Let $\mathfrak{S}$ be the Cartan subalgebra of $\mathfrak{L}$ fixed pointwise by $\tilde{\eta}$. Now $\eta$ stabilizes $\mathfrak{\Im}_{1}$ and $\mathfrak{\Im}_{2}$. To see that this is true, one observes that since $\mathfrak{\Im}_{i}$ is an ideal, $\left[\mathfrak{\Im}_{i}, \mathfrak{L}\right]=\mathfrak{\Im}_{i} \mathfrak{L} \subseteq \mathfrak{\Im}_{i}$; in particular, $\left[\mathfrak{\Im}_{i}, \mathfrak{S}\right] \subseteq \mathfrak{\Im}_{i}$, so that $\mathfrak{\Im}_{i}$ is a sum of root spaces of $\mathfrak{\Re}$. Since $\tilde{\eta}$ stabilizes the root spaces of $\mathfrak{\Re}, \eta=\tilde{\eta} \mid \mathfrak{\Im}$ stabilizes $\mathfrak{\Im}_{i}$. Moreover, the hypotheses of the Lemma apply to $\mathfrak{\Im}_{i}$ and $\eta \mid \mathfrak{\Im}_{i}$. Indeed, Der $\mathfrak{I}$ $=\operatorname{Der} \mathfrak{\Im}_{1} \oplus \operatorname{Der} \mathfrak{\Im}_{2}$ so that $\eta \mid \mathfrak{\Im}_{i}$ commutes with every derivation of $\mathfrak{\Im}_{i}$. Also $\mathfrak{S}=\mathfrak{S}_{1} \oplus \mathfrak{S}_{2}$, where $\mathfrak{S}_{i}=\mathfrak{S}_{2} \cap \mathfrak{Q}_{i}$ is a Cartan subalgebra of $\mathfrak{L}_{i}$; and $\left(\eta \mid \mathfrak{S}_{i}\right)^{\sim}=\tilde{\eta} \mid \mathfrak{\Re}_{i}$ fixes $\mathfrak{S}_{i}$ pointwise. By induction we conclude that $\eta \mid \mathfrak{\Im}_{i}$ is in Aut $_{0} \mathfrak{\Im}_{i}$. Since the direct product of irreducible groups is irreducible, $\eta \in \mathrm{Aut}_{0} \Im_{1} \times \mathrm{Aut}_{0} \Im_{2} \subseteq G_{0}$.

We assume henceforth that $\Im$ is simple. Since $\Im \subseteq \mathfrak{I}$ is a sum of root spaces, $\eta$ is a diagonal linear transformation of $\mathfrak{\Im}$ (with respect to a basis of $\mathfrak{\Im}$ consisting of root vectors). Let $\lambda$ be an eigenvalue of $\eta$. Then the $\lambda$-eigenspace $\Im_{\lambda}(\eta)$ is invariant under all derivations of $\Im$. Indeed, if $x \eta=\lambda x$ and $D \in \mathfrak{D}$ then $(x D) \eta=(x \eta) D=\lambda(x D)$. Now if $\eta=1$ we are clearly done. So assume $\eta \neq 1$. Then $\eta$ has an eigenvalue $\lambda \neq 1$. $\Im_{\lambda}(\eta)$ is thus a subspace of $\mathfrak{\Im}$ invariant under $\mathscr{D}$ and disjoint from $\Phi 1$. By a result of Helwig [4, Theorem 4, p. 389], either the dimension of $\mathfrak{\Im}$ is 3 , or $\mathfrak{\Im}_{\lambda}(\eta)=\mathfrak{\Im}^{\prime}$, the subspace of $\mathfrak{\Im}$ spanned by all associators. We note that Helwig's proof does not use the classification of simple Jordan algebras.

Suppose then that $\operatorname{dim} \mathfrak{\Im}=3$. Then as we have seen $\mathfrak{\Im}$ is the Jordan algebra of a two-dimensional vector space $\mathfrak{B}$ with a symmetric nondegenerate bilinear form $\left(\right.$, ). Let $v_{1}, v_{2}$ be the basis of $\mathfrak{B}$ given in the Lemma preceding Proposition 16. Now $\eta$ (indeed any automorphism) stabilizes $\mathfrak{B}=\mathfrak{S}^{\prime}$ and fixes 1 . If the matrix of $\eta$ on $\mathfrak{B}$ is $\left(\begin{array}{ll}a & b \\ c & d\end{array}\right)$, then from the fact that $\eta$ commutes with derivations we see that

i.e.

$$
\left(\begin{array}{rr}
1 & 0 \\
0 & -1
\end{array}\right)\left(\begin{array}{ll}
a & b \\
c & d
\end{array}\right)=\left(\begin{array}{ll}
a & b \\
c & d
\end{array}\right)\left(\begin{array}{rr}
1 & 0 \\
0 & -1
\end{array}\right),
$$

$$
\left(\begin{array}{rr}
a & b \\
-c & -d
\end{array}\right)=\left(\begin{array}{ll}
a & -b \\
c & -d
\end{array}\right) \text {. }
$$


This means that $b=c=0$. Also $v_{1} \eta \cdot v_{2} \eta=1$ implies that $a d=1$. So $\eta \mid \mathfrak{B}$ has the matrix $\left(\begin{array}{cc}a & 0 \\ 0 & a\end{array}-1\right)$. Now if $\lambda \neq 0$ is any scalar, the linear transformation $\eta_{\lambda}$ of $\mathfrak{J}$ which maps $1 \rightarrow 1, v_{1} \rightarrow \lambda v_{1}, v_{2} \rightarrow \lambda^{-1} v_{2}$ is an automorphism of $\Im$. The set of all $\eta_{\lambda}$ is an irreducible set since it is the image of $\Phi-\{0\}$ under the rational map $\lambda \mapsto \eta_{\lambda}$. Hence all $\eta_{\lambda}$ are in Aut $_{0} \mathfrak{\Im}$. This applies in particular to the given $\eta$.

We can now assume that $\lambda \neq 1$ is an eigenvalue of $\eta$ and that $\Im_{\lambda}(\eta)=\Im^{\prime}$, where $\mathfrak{\Im}=\Phi 1 \oplus \mathfrak{\Im}^{\prime}$ (since $\mathfrak{\Im}$ is central simple [4]). Thus $\mathfrak{\Im}=\Phi 1 \oplus \mathfrak{\Im}_{\lambda}(\eta)$, and $\eta$ has only 1 and $\lambda$ as eigenvalues. If $x$ and $y$ are eigenvectors for $\lambda$ then $x . y$ is zero or is an eigenvector for $\lambda^{2}$. This means that one of three things happens: $\lambda^{2}=1, \lambda^{2}=\lambda$, or $\Im^{\prime} \cdot \Im^{\prime}$

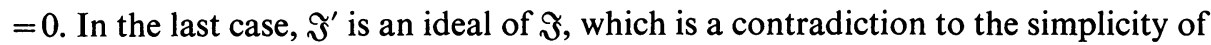
$\Im$ Since $\lambda \neq 1$ the middle case is impossible and the first implies that $\lambda=-1$.

This means that if $x, y \in \mathfrak{\Im}^{\prime}$ then $x . y$ is an eigenvector for 1 , i.e. is in $\Phi 1$. For each $x, y \in \Im^{\prime}$ let $(x, y) \in \Phi$ be such that $x . y=(x, y) 1$. It then follows that $\mathfrak{I}$ is the Jordan algebra of the vector space $\mathfrak{I}^{\prime} \equiv \mathfrak{B}$ equipped with the bilinear form ( , ), and that $\eta$ is the automorphism $\mu 1+v \mapsto \mu 1-v, \mu \in \Phi, v \in \mathfrak{B}$.

The action of $\tilde{\eta}$ on $\mathfrak{Q}$ is as follows: $\tilde{\eta}: R_{1} \rightarrow R_{1}, R_{x} \rightarrow-R_{x}$ if $x \in \mathfrak{B}, D \rightarrow D$ if $D \in \mathcal{D}$. Since $\tilde{\eta}$ fixes a Cartan subalgebra $\mathfrak{S}$ of $\mathfrak{R}$, it follows that $\mathfrak{S} \subseteq \Phi R_{1} \oplus \mathfrak{D}$. This implies (since $R_{1}$ is in the center of $\Phi R_{1} \oplus \mathfrak{D}$ ) that $\mathfrak{S}_{\mathfrak{Z}}=\Phi R_{1} \oplus \mathfrak{B}$ where $\mathfrak{B}$ is a Cartan subalgebra of $\mathfrak{D}$. We show that this situation is impossible if $\operatorname{dim} \mathfrak{B}$ is odd. In any event the derivations of $\mathfrak{\Im}$ are the linear transformations of $\mathfrak{\Im}$ which send 1 to 0 and which on $\mathfrak{B}$ are skew with respect to the bilinear form. If $\operatorname{dim} \mathfrak{B}$ is odd we can choose a basis $v_{0}, v_{1}, \ldots, v_{2 l}$ of $\mathfrak{B}$ with respect to which the matrix of the form is

$$
\left(\begin{array}{lll}
1 & 0 & 0 \\
0 & 0 & I_{l} \\
0 & I_{l} & 0
\end{array}\right)
$$

Then a Cartan subalgebra $\mathfrak{M}$ of $\mathfrak{D}$ consists of transformations which annihilate 1 and on $\mathfrak{B}$ have matrices of the form

$$
\left(\begin{array}{rrr}
0 & 0 & 0 \\
0 & A & 0 \\
0 & 0 & -A
\end{array}\right),
$$

where $A$ is an $l \times l$ diagonal matrix [8, pp. 138-139]. Now $\mathfrak{W}$ annihilates the subspace $\Phi 1 \oplus \Phi v_{0}$ of $\Im$ so that the Cartan subalgebra of $\mathfrak{L}$ containing $\mathfrak{W}$ contains $R_{\Phi 1 \oplus \Phi v_{0}}$ (see Proposition 1). Thus rank $\mathfrak{L} \geqq$ rank $\mathfrak{D}+2$, whereas the hypotheses on $\eta$ would imply that rank $\mathfrak{Q}=\operatorname{rank} \mathfrak{D}+1$.

Finally we show that if $\operatorname{dim} \mathfrak{B}$ is even, $\eta$ is indeed in Aut $_{0} \Im$. We choose a basis $v_{1}, \ldots, v_{n}$ of $\mathfrak{B}$ such that $\left(v_{i}, v_{j}\right)=\delta_{i j}$. For each $i, e_{i}=\frac{1}{2}\left(1+v_{i}\right)$ is a primitive idempotent of $\Im$ : since $v_{i}^{2}=1, e_{i}$ is an idempotent, and since $\Im$ has rank 2 , every idempotent of $\Im$ not equal to 0 or to 1 is primitive. Now it is known (see Propositions 
1 and 3 of the Appendix) that if $e, f$ are primitive idempotents of $\Im$, then $U_{2 e-1} U_{2 f-1}$ $\in \mathrm{Aut}_{0} \Im$. Applying this here, and noting that $U_{2 e_{i}-1}=U_{v_{i}}$, we see from the fact that $n$ is even that $U_{v_{1}} U_{v_{2}} \cdots U_{v_{n}} \in \operatorname{Aut}_{0} \Im$. Now $1 U_{v_{i}}=1, v_{i} U_{v_{i}}=v_{i}^{3}=\left(v_{i}, v_{i}\right) v_{i}=v_{i}$ and if $i \neq j$ then $v_{j} U_{v_{i}}=2\left(v, \cdot v_{i}\right) \cdot v_{i}-v_{j} \cdot v_{i}^{2}=0-v_{j}=-v_{j}$. This implies that $1 U_{v_{1}} \cdots U_{v_{n}}$ $=1$ and that for each $i, v_{i} U_{v_{1}} \cdots U_{v_{n}}=(-1)^{n-1} v_{i}=-v_{i}$. Thus $\eta=U_{v_{1}} \cdots U_{v_{n}}$, and $\eta \in$ Aut $_{0} \Im$, as desired.

This theorem, together with the preceding considerations, allows us to identify the components of $G$. We denote by $W\left(\mathfrak{D}^{\prime}\right)$ the Weyl group of $\mathfrak{D}^{\prime}$ and by $W\left(\Sigma_{\varepsilon}\right)$ the Weyl group of $\Sigma_{\varepsilon}$. We define a map $i$ from $W\left(\Sigma_{\varepsilon}\right)$ to $G / G_{0}$ as follows: let $u \in W\left(\Sigma_{\varepsilon}\right)$. Then by Steinberg's theorem there is a unique $v$ in the Weyl group of $\mathfrak{L}^{\prime}$ such that $v$ commutes with $\varepsilon^{*}$ and $v \mid \mathfrak{\mathfrak { B } ^ { * }}=u$. By Proposition 11 there is a unique $w$ in the Weyl group of $\Re$ such that $w$ permutes the roots $\rho$ of $\Re$ satisfying $\rho\left(R_{1}\right)=1$ and $w \mid\left(\mathfrak{S}_{1}^{*}\right)^{\wedge}=\hat{v}$. By Proposition $12 w$ commutes with $\varepsilon^{*}$. By Theorem 2(b) there is a $\zeta \in G$ such that $\tilde{\zeta}$ stabilizes $\mathfrak{H}$ and $\tilde{\zeta}^{*}=w$. We let $i(u)=\zeta G_{0}$.

This map is well defined. Suppose $\zeta_{1}, \zeta_{2} \in G$ both stabilize $\mathfrak{S}$ and satisfy $\tilde{\zeta}_{i}^{*}=w$ $(i=1,2)$. Then $\left(\tilde{\zeta}_{1} \tilde{\zeta}_{2}^{-1}\right)^{*}=1$. We show that if $\tilde{\zeta} \in \tilde{G}$ stabilizes $\mathfrak{H}$ and satisfies $\tilde{\zeta}^{*}=1$, then $\zeta \in G_{0}$; this will obviously be sufficient. It is clear that $\tilde{\zeta} \in \mathrm{Aut}_{0} \Re$. If $w, v, u$ are as in the paragraphs preceding Proposition 24 and following Proposition 25 (that is, $\left.\tilde{\zeta}^{*}=w, w\left|\left(\mathfrak{S}_{1}^{*}\right)^{\wedge}=\hat{v}, \bar{u}\right|\left(\mathfrak{W}_{1}^{*}\right)^{-}=v \mid\left(\mathfrak{W}_{1}^{*}\right)^{-}\right)$then clearly $w=1$ (acting on $\mathfrak{S}^{*}$ ), hence $v=1$ (acting on $\mathfrak{S}_{1}^{*}$ ), hence $u=1$ (acting on $\mathfrak{S}_{1}^{*}$ ). The remarks following Proposition 25 show that $\zeta$ stabilizes $\mathfrak{W}_{1}$ and $\check{\zeta}^{*}=u$. Hence $\check{\zeta}^{*}=1$ and $\check{\zeta} \in \mathrm{Aut}_{0} \mathfrak{D}^{\prime}$. A similar argument shows that the dual of $\tilde{\zeta} \mid \Subset$ can be identified with $v \mid \mathfrak{F}^{*}=1$, so that $\tilde{\zeta}$ fixes $\&$ pointwise. Theorem 3 now implies that $\zeta \in G_{0}$.

The map $i$ defined above is an anti-homomorphism. For suppose $u_{1}, u_{2} \in W\left(\Sigma_{\varepsilon}\right)$ and let $v_{i}, w_{i}, \zeta_{i}(i=1,2)$ be as above. Then $v_{1} v_{2}$ is in the Weyl group of $\mathfrak{R}^{\prime}$, commutes with $\varepsilon^{*}$ and satisfies $v_{1} v_{2} \mid \mathfrak{I S}^{*}=u_{1} u_{2}$. Also $w_{1} w_{2}$ satisfies $w_{1} w_{2} \mid\left(\mathfrak{S}_{1}^{*}\right)^{\wedge}=\hat{v}_{1} \hat{v}_{2}$ $=\left(v_{1} v_{2}\right)^{\wedge}$. Finally $\left(\tilde{\zeta}_{2} \tilde{\zeta}_{1}\right)^{*}=\tilde{\zeta}_{1}^{*} \tilde{\zeta}_{2}^{*}=w_{1} w_{2}$ so $i\left(u_{1} u_{2}\right)=\zeta_{2} \zeta_{1} G_{0}=\zeta_{2} G_{0} \zeta_{1} G_{0}=i\left(u_{2}\right) i\left(u_{1}\right)$.

We show that the kernel of $i$ is $\left(W\left(\mathfrak{D}^{\prime}\right)\right)^{-}$. Suppose $u \in W\left(\mathfrak{D}^{\prime}\right)$. Then choose $v, w, \zeta$ so that $v\left|\mathfrak{R}^{*}=\bar{u}, w\right|\left(\mathfrak{S}_{1}^{*}\right)^{\wedge}=\hat{v}, \tilde{\zeta}^{*}=w$, as above. Of course $\tilde{\zeta} \in \mathrm{Aut}_{0} \mathfrak{\AA}$ since $w$ is in the Weyl group of $\AA$. We have seen that $\check{\zeta}^{*}=u$, so that $\breve{\zeta} \in \mathrm{Aut}_{0} \mathfrak{D}^{\prime}$. As was remarked above, the dual of $\tilde{\zeta} \mid \mathfrak{F}$ can be identified with $\bar{u} \mid \mathfrak{E}^{*}$, which is 1 by the definition of $\bar{u}$. Hence Theorem 3 implies that $\zeta \in G_{0}$; we have shown that the kernel of $i$ contains $\left(W\left(D^{\prime}\right)\right)^{-}$. Conversely, suppose $u \in W\left(\Sigma_{\varepsilon}\right), i(u)=1$. Choose

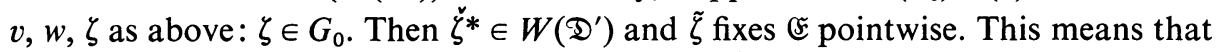
$u$ fixes $\mathfrak{F}^{*}$ pointwise and on $\left(\mathfrak{W}_{1}^{*}\right)^{-}$agrees with $\left(\check{\zeta}^{*}\right)^{-}$; i.e. $u \in\left(W\left(\mathfrak{D}^{\prime}\right)\right)^{-}$.

We can therefore define an injective homomorphism $j$ of $W\left(\Sigma_{\varepsilon}\right) /\left(W\left(\mathfrak{D}^{\prime}\right)\right)^{-}$into $G / G_{0}$ by the formula $j\left(u\left(W\left(\mathfrak{D}^{\prime}\right)\right)^{-}\right)=i\left(u^{-1}\right)$. We define a homomorphism $p: G / G_{0}$ $\rightarrow \Gamma / \Gamma_{0}$ as follows: $p\left(G_{i}\right)$ is that component of $\Gamma$ which contains $G_{i}$. Our principal result is

THEOREM 4. The sequence

$$
0 \longrightarrow W\left(\Sigma_{\varepsilon}\right) /\left(W\left(\mathfrak{D}^{\prime}\right)\right)^{-} \stackrel{j}{\longrightarrow} G / G_{0} \stackrel{p}{\longrightarrow} \Gamma / \Gamma_{0} \longrightarrow 0
$$


is a split exact sequence. Consequently the number of components of $G$ is the number of components of $\Gamma$ times the index of $\left(W\left(\mathfrak{D}^{\prime}\right)\right)^{-}$in $W\left(\Sigma_{\varepsilon}\right)$.

Proof. To prove exactness, we need only show that the image of $j$ (i.e., the image of $i$ ) is the kernel of $p$, and that $p$ is surjective. If $u \in W\left(\Sigma_{\varepsilon}\right)$ and $v, w, \zeta$ are as above, then $\tilde{\zeta}^{*}=w$ is in the Weyl group of $\Re$ and hence, by Theorem 1(c), $\zeta \in \Gamma_{0}$. This means that $p(i(u))=p\left(\zeta G_{0}\right)=\zeta \Gamma_{0}=\Gamma_{0}$. Conversely, suppose $G_{i}$ is a component of $G$ with $G_{i} \subseteq \Gamma_{0}$. Choose $\zeta \in G_{i}$ so that $\tilde{\zeta}$ stabilizes $\mathfrak{H}$ (Theorem 2(a)). Let $w=\tilde{\zeta}^{*}$; $w$ is in the Weyl group of $\Re$ (since $\zeta \in \Gamma_{0}$ ) and commutes with $\varepsilon^{*}$ (since $\zeta \in G$ ). By Proposition $11 w \mid\left(\mathfrak{S}_{1}^{*}\right)^{\wedge}=\hat{v}$ where $v$ is in the Weyl group of $\mathfrak{L}^{\prime}$ and commutes with $\varepsilon^{*}$. Steinberg's theorem then implies that $u=v \mid \mathfrak{B S}^{*}$ is in $W\left(\Sigma_{\varepsilon}\right)$. Clearly $i(u)=G_{i}$.

We now define a homomorphism $q$ from $\Gamma / \Gamma_{0}$ to $G / G_{0}$ and prove that $p\left(q\left(\Gamma_{i}\right)\right)=$ $\Gamma_{i}$ for all components $\Gamma_{i}$. This will prove that $p$ is surjective and that the sequence splits. Choose $\eta \in \Gamma_{i}$ so that $\tilde{\eta}$ stabilizes $\mathfrak{S}$ and $\tilde{\eta}^{*}$ stabilizes our fixed simple system of roots $\left\{\rho_{1}, \ldots, \rho_{r}, \hat{\alpha}_{1}, \ldots, \hat{\alpha}_{l}\right\}$; here we use the Corollary to Theorem 1 . Then by Proposition $23 \tilde{\eta}^{*}$ commutes with $\varepsilon^{*}$. We conclude from Theorem 2(b) that there is a $\zeta \in G$ such that $\tilde{\zeta}$ stabilizes $\mathscr{S}$ and $\tilde{\zeta}^{*}=\tilde{\eta}^{*}$, i.e. $\left(\tilde{\zeta} \tilde{\eta}^{-1}\right)^{*}=1$, so that $\zeta_{\eta}{ }^{-1} \in \Gamma_{0}$ by Theorem 1(c). We have shown that for each $\Gamma_{i}$ there is a $\zeta \in G \cap \Gamma_{i}$ such that $\tilde{\zeta}$ stabilizes $\mathfrak{H}$ and $\tilde{\zeta}^{*}$ stabilizes our simple system of roots. We let $q\left(\Gamma_{i}\right)=\zeta G_{0}$. This map is well defined: if $\zeta_{1}, \zeta_{2} \in G \cap \Gamma_{i}$ both have the above property then $\tilde{\zeta}_{1} \tilde{\zeta}_{2}^{-1} \in \tilde{\Gamma}_{0}$ and $\left(\tilde{\zeta}_{1} \tilde{\zeta}_{2}^{-1}\right)^{*}$ stabilizes the simple system. By Theorem $1(\mathrm{c})\left(\tilde{\zeta}_{1} \tilde{\zeta}_{2}^{-1}\right)^{*}$ is in the Weyl group of $\AA$, hence equals 1 . By our remark above, $\tilde{\zeta}_{1} \tilde{\zeta}_{2}^{-1} \in G_{0}$.

The map $q$ is a homomorphism: if $\zeta_{1} \in G \cap \Gamma_{1}, \zeta_{2} \in G \cap \Gamma_{2}$ are such that $\tilde{\zeta}_{i}$ stabilize $\mathfrak{H}$ and $\tilde{\zeta}_{i}^{*}$ stabilize the simple system, then $\zeta_{1} \zeta_{2} \in G \cap \Gamma_{1} \Gamma_{2}$ has the property that $\tilde{\zeta}_{1} \tilde{\zeta}_{2}$ stabilizes $\mathfrak{S}$ and $\left(\tilde{\zeta}_{1} \tilde{\zeta}_{2}\right)^{*}$ stabilizes the simple system. I.e. $q\left(\Gamma_{1} \Gamma_{2}\right)=$ $\zeta_{1} \zeta_{2} G_{0}=\zeta_{1} G_{0} \zeta_{2} G_{0}=q\left(\Gamma_{1}\right) q\left(\Gamma_{2}\right)$. Finally, if $\Gamma_{i}$, $\zeta$ are as above, $p\left(q\left(\Gamma_{i}\right)\right)=p\left(\zeta G_{0}\right)=$ $\zeta \Gamma_{0}=\Gamma_{i}$ since $\zeta \in \Gamma_{i}$.

The second sentence of the theorem clearly follows from exactness of the sequence.

4. Fixed points. If $\eta$ is a linear transformation of a vector space $\mathfrak{B}$ over $\Phi$, by $\mathfrak{B}_{\lambda}(\eta)$ we mean $\left\{v \in \mathfrak{B} \mid v(\eta-\lambda 1)^{k}=0\right.$ for some integer $\left.k\right\} . \mathfrak{B}_{\lambda}(\eta)$ is the $\lambda$-eigenspace of $\eta$, and will be written $\mathfrak{B}_{\lambda}$ if $\eta$ is understood. Suppose $\mathfrak{B}$ is an algebra and $\eta$ an automorphism of $\mathfrak{B}$. Then $\mathfrak{B}_{\lambda} \mathfrak{B}_{\mu} \subseteq \mathfrak{B}_{\lambda \mu}$; in particular, $\mathfrak{B}_{\lambda} \mathfrak{B}_{\mu}=0$ if $\lambda \mu$ is not an eigenvalue of $\eta$. Also, if $\mathfrak{B}$ has an identity, then $\mathfrak{B}_{1}$ is a subalgebra of $\mathfrak{B}$ containing 1 .

If $G_{i}$ is an algebraic component of $G=$ Aut $\mathfrak{~}$, then by the index of $G_{i}$ we mean the minimum dimension of $\Im_{1}(\eta)$ as $\eta$ ranges over $G_{i}$. If $\eta \in G_{i}$ and $\operatorname{dim} \Im_{1}(\eta)$ equals the index of $G_{i}$, then $\eta$ is called regular. In these definitions we follow Jacobson [9], as outlined in the Introduction. As in the case of Lie algebras, the regular elements of $G_{i}$ form a nonempty Zariski open subset of $G_{i}[9$, p. 304].

Proposition 27. If $\eta \in G$ then $\Im_{1}(\eta)$ is a semisimple subalgebra of $\mathfrak{~}$. Also $\mathfrak{R}=\oplus \sum_{\lambda \neq 1} \mathfrak{\Im}_{\lambda}(\eta)$ is the orthogonal complement of $\mathfrak{\Im}_{1}(\eta)$ with respect to the generic

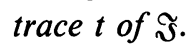


Proof. Let $\eta_{s}$ be the semisimple part of $\eta$-i.e. if $z \in \Im_{\lambda}$ then $z \eta_{s}=\lambda z$. Then $\eta_{s}$ is also an automorphism. If $z \in \Im_{1}, w \in \Im_{\lambda}, \lambda \neq 1$, then

$$
t(z, w)=t(z . w)=t\left((z . w) \eta_{s}\right)=t\left(z \eta_{s}, w \eta_{s}\right)=\lambda t(z, w) .
$$

Thus $\lambda \neq 1$ implies that $t(z, w)=0$. This proves that $t\left(\Im_{1}, \mathfrak{R}\right)=0$. Since $\operatorname{dim} \mathfrak{R}=$ $\operatorname{dim} \mathfrak{\Im}-\operatorname{dim} \mathfrak{\Im}_{1}$ and since the generic trace is nondegenerate (see [10, p. 353] for a proof of this independent of the classification theory), it follows that $\mathfrak{R}=\mathfrak{F}_{1}^{1}$.

From the above proven fact we see that $t$ restricted to $\Im_{1}$ is a nondegenerate associative form. We can use Dieudonné's theorem [8, p. 71] to prove that $\Im_{1}$ is semisimple, provided we show that $\mathfrak{\Im}_{1}$ contains no ideals of square zero. If $\mathfrak{B}$ is such an ideal, let $z \in \Im_{1}, w \in \mathfrak{B}$. Then $t(z, w)=t(z . w)=0$ since $z . w \in \mathfrak{B}$ and hence $(z . w)^{2}=0$ (here we use the fact that $t$ is zero on nilpotent elements of $\Im$ ). But now we see that $\mathfrak{B} \subseteq \Im_{2}^{\frac{1}{2}} \cap \Im_{1}=0$.

In the following theorem, we use results on idempotents which are proven in the Appendix.

THEOREM 5. Let $\eta$ be a regular automorphism of $\Im$. Then $\Im_{1}=\Im_{1}(\eta)$ is an associative semisimple subalgebra of $\Im$, i.e. a direct sum of fields.

Proof. Suppose $\mathfrak{\Im}_{1}$ is not a direct sum of fields. By Proposition 27 it is a direct sum of simple algebras, one of which is not a field. By standard results on reduced simple Jordan algebras $\left[10\right.$, p. 202], $\Im_{1}$ contains primitive orthogonal idempotents $e, f$ such that $e$ and $f$ are connected-indeed, since the field is algebraically closed, strongly connected. Thus we have $e, f, a \in \mathfrak{\Im}_{1}$ with $e . a=f . a=\frac{1}{2} a$ and $a^{2}=e+f$. Let $\mathfrak{B}=\Phi e+\Phi f+\Phi a ; \mathfrak{B}$ is a three-dimensional simple Jordan algebra. Let $I(\mathfrak{B})$ be the set of primitive idempotents of $\mathfrak{B}$. Since $\mathfrak{B}$ is the Jordan algebra of a vector space with a nondegenerate bilinear form, $I(\mathfrak{B})$ is an irreducible algebraic subset of $\mathfrak{B}$ (see Proposition 3 of the Appendix). Moreover, $e, f$, and $\frac{1}{2}(e+f+a)$ are in $I(\mathfrak{B})$, since they are idempotents unequal to 0 or 1 . We define a polynomial map $\zeta: I(\mathfrak{B}) \rightarrow G$ as follows:

$$
\zeta(g)=U_{2 e-1} U_{2 g-1} \eta_{s} \quad \text { for } g \in I(\mathfrak{B}) .
$$

Here $\mathfrak{N}_{s}$ is the semisimple part of $\eta$. By Proposition 1 of the Appendix, $U_{2 e-1} U_{2 g-1} \in$ $G_{0}$. Also since the characteristic of $\Phi$ is zero, any unipotent automorphism of $\mathfrak{\Im}$ is in $G_{0}$, so that in particular $\eta_{s}$ and $\eta$ belong to the same component of $G$. Thus $\zeta(I(\mathfrak{B})) \subseteq G_{0} \eta$.

Let $\mathfrak{N}$ be as in Proposition 27. If $x \in \Im_{1}$ and $g \in I(\mathfrak{B})$ then $x U_{2 g-1}=8(x . g) . g-$ $8 x . g+x \in \Im_{1}$ (as in the Appendix). This shows that $\Im_{1} \zeta(g) \subseteq \Im_{1}$; since automorphisms of $\Im$ are orthogonal with respect to the generic trace, $\mathfrak{R} \zeta(g)=\Im_{1}^{1} \zeta(g) \subseteq \mathfrak{R}$. I.e. $\zeta(g)$ stabilizes $\Im_{1}$ and $\mathfrak{R}$ for any $g \in I(\mathfrak{B})$.

We now define two polynomial functions on $I(\mathfrak{B})$ : if $g \in I(\mathfrak{B})$, then $E(g)=$ $\operatorname{det}[(\zeta(g)-I) \mid \Re]$ and $F(g)=(\zeta(g)-I)^{k} \mid \Im_{1}$, where $k=\operatorname{dim} \Im_{1}$ and $I$ is the identity linear transformation of $\mathfrak{\Im}$. Thus $E: I(\mathfrak{B}) \rightarrow \Phi$ and $F: I(\mathfrak{B}) \rightarrow$ 
$\operatorname{Hom}_{\Phi}\left(\Im_{1}, \Im_{1}\right)$. We claim that neither $E$ nor $F$ is the zero function. Indeed, $E(e)=\operatorname{det}[(\zeta(e)-I) \mid \mathfrak{N}]=\operatorname{det}\left[\left(\eta_{s}-I\right) \mid \mathfrak{N}\right] \neq 0$, since 1 is not an eigenvalue of $\eta \mid \mathfrak{R}$. Let $g=\frac{1}{2}(e+f+a)$; then $F(g) \neq 0$. To see this, we note that $a . g=\frac{1}{2}(a / 2+a / 2+e+f)$ $=\frac{1}{2}(a+e+f)=g$, which means that $a U_{2 g-1}=8(a . g) \cdot g-8 a \cdot g+a=8 g \cdot g-8 g+a=a$. Now $a U_{2 e-1}=8(a . e) . e-8 a . e+a=2 a-4 a+a=-a$. Hence $a \zeta(g)=a U_{2 e-1} U_{2 g-1} \eta_{s}$ $=-a \eta_{s}=-a$. So $a(\zeta(g)-I)=-2 a$ and finally $a(\zeta(G)-I)^{k}=(-2)^{k} a \neq 0$. This shows that $F \neq 0$.

Since $I(\mathfrak{B})$ is irreducible, we can choose $h \in I(\mathfrak{B})$ so that $E(h) \neq 0$ and $F(h) \neq 0$. Since $E(h) \neq 0, \zeta(h)-I$ is nonsingular on $\mathfrak{N}$ and thus $\Im_{1}(\zeta(h)) \subseteq \Im_{1}(\eta)$. Since $F(h) \neq 0$, $\zeta(h)-I$ is not nilpotent on $\Im_{1}$, which means that $\Im_{1}(\zeta(h))$ is properly contained in $\Im_{1}$. But $\zeta(h) \in G_{0} \eta$, a contradiction to the fact that $\eta$ is regular.

We now work toward showing the converse of Theorem 5. In Propositions 28 through $31 \eta$ is an automorphism of $\Im$ such that $\mathfrak{\Im}_{1}(\eta)$ is associative, i.e. $\mathfrak{\Im}_{1}(\eta)=$ $\Phi e_{1} \oplus \cdots \oplus \Phi e_{k}$, where $e_{1}, \ldots, e_{k}$ are orthogonal idempotents and $e_{1}+\cdots+e_{k}=1$. As before $\mathfrak{R}=\oplus \sum_{\lambda \neq 1} \mathfrak{\Im}_{\lambda}(\eta)$. Let $\mathfrak{D}_{0}=\left\{D \in \mathfrak{D} \mid \mathfrak{\Im}_{1} D=0\right\}$.

Proposition 28. $\eta$ fixes $\Im_{1}$ pointwise.

Proof. $\eta \mid \Im_{1}$ is unipotent. It permutes the idempotents $e_{1}, \ldots, e_{k}$ and thus has finite order. The group algebra of the cyclic group generated by $\eta \mid \Im_{1}$ is semisimple (by Maschke's theorem and since $\Phi$ has characteristic zero) and commutative, hence contains no nilpotent elements. Thus the nilpotent part of $\eta \mid \Im_{1}$ is 0 , and $\eta \mid \Im_{1}=1$.

Proposition 29. Every element $D \in \mathfrak{D}$ can be written in the form $D=D_{1}+D_{2}-$ $\eta^{-1} D_{2} \eta$, where $D_{1} \in \mathfrak{D}_{0}$ and $D_{2} \in \mathfrak{D}$.

Proof. Let $\mathfrak{\Im}=\oplus \sum_{i \leqq j} \mathfrak{\Im}_{i j}$ be the Peirce decomposition of $\mathfrak{J}$ with respect to the idempotents $e_{1}, \ldots, e_{k}$. Now $e_{i} D=e_{i}^{2} D=2 e_{i} . e_{i} D$, so that $e_{i} D \in \Im_{1 / 2}\left(e_{i}\right)=$ $\bigoplus \sum_{n \neq i} \Im_{i n}$. Choose $a_{i n} \in \Im_{i n}(n \neq i)$ so that $e_{i} D=\sum_{n \neq i} a_{i n}$. Then if $i \neq j$,

$$
0=\left(e_{1} \cdot e_{j}\right) D=e_{i} D \cdot e_{j}+e_{i} \cdot e_{j} D=\sum_{n \neq i} a_{i n} \cdot e_{j}+e_{i} \cdot \sum_{n \neq j} a_{j n}=\frac{1}{2} a_{i j}+\frac{1}{2} a_{j i} \text {. }
$$

Thus $a_{i j}=-a_{j i}$. Now let

Then for each $i$,

$$
E=\sum_{n<m} 4\left[R_{e_{n}}, R_{a_{n m}}\right] \in \mathfrak{D}
$$

$$
\begin{aligned}
e_{i} E & =\sum_{n<m} 4\left(e_{i} \cdot e_{n}\right) \cdot a_{n m}-\sum_{n<m} 4\left(e_{i} \cdot a_{n m}\right) \cdot e_{n} \\
& =\sum_{i<m} 2 a_{i m}-\sum_{i<m} a_{i m}-\sum_{n<i} a_{n i} \\
& =\sum_{i<m} a_{i m}-\sum_{m<i} a_{m i}=\sum_{m \neq i} a_{i m}=e_{i} D .
\end{aligned}
$$

So $D_{1}=D-E \in \mathfrak{D}_{0}$. To conclude, we need only show that $E$ is of the form 
$D_{2}-\eta^{-1} D_{2} \eta$. For this we need only show that each $\left[R_{e_{n}}, R_{a_{n m}}\right]$ is of that form. Now if $i \neq m, i \neq n$ then $t\left(e_{i}, a_{n m}\right)=t\left(e_{i}, a_{n m}\right)=t(0)=0$; also $t\left(e_{n}, a_{n m}\right)=t\left(e_{n}^{2}, a_{n m}\right)=$ $t\left(e_{n}, e_{n} . a_{n m}\right)=\frac{1}{2} t\left(e_{n}, a_{n m}\right)=0$ and similarly $t\left(e_{m}, a_{n m}\right)=0$. From this we see, since $\mathfrak{N}=\Im_{1}^{\frac{1}{1}}$, that $a_{n m} \in \mathfrak{N}$. Now $1-\eta$ stabilizes $\mathfrak{N}$ and is nonsingular on $\mathfrak{N}$, hence surjective on $\mathfrak{R}$. Choose $b \in \mathfrak{N}$ so that $b-b \eta=a_{n m}$. Then

$$
\begin{aligned}
{\left[R_{e_{n}}, R_{a_{n m}}\right] } & =\left[R_{e_{n}}, R_{b}\right]-\left[R_{e_{n}}, R_{b \eta}\right] \\
& =\left[R_{e_{n}}, R_{b}\right]-\left[R_{e_{n} \eta}, R_{b \eta}\right] \\
& =\left[R_{e_{n}}, R_{b}\right]-\left[R_{e_{n}} \tilde{\eta}, R_{b} \tilde{\eta}\right] \\
& =\left[R_{e_{n}}, R_{b}\right]-\left[R_{e_{n}}, R_{b}\right] \tilde{\eta} \\
& =\left[R_{e_{n}}, R_{b}\right]-\eta^{-1}\left[R_{e_{n}}, R_{b}\right] \eta
\end{aligned}
$$

Let $H=\left\{\tau \in G \mid \Im_{1} \tau \subseteq \Im_{1}\right\}$. $H$ is clearly an algebraic group. Since $H \subseteq G$, it follows that $H_{0} \subseteq G_{0}$.

Proposition 30. (a) The elements of $H_{0}$ fix $\Im_{1}$ pointwise.

(b) The Lie algebra of $H_{0}$ is $\mathfrak{D}_{0}$.

Proof. (a) Let $H_{1}=\left\{\tau \in H_{0} \mid x \tau=x\right.$ for all $\left.x \in \mathfrak{\Im}_{1}\right\}$. Then $H_{1}$ is a normal algebraic subgroup of $H_{0}$. Moreover, it has finite index in $H_{0}$. Indeed, since $\Im_{1}$ is a direct sum of fields, Aut $\Im_{1}$ is finite. Two elements of $H_{0}$ are in the same coset of $H_{1}$ if and only if they agree on $\Im_{1}$; since there are only a finite number of possible actions on $\Im_{1},\left[H_{0}: H_{1}\right]$ is finite. This means that the component of the identity of $H_{1}$ has finite index in $H_{0}$ and thus is the component of the identity of $H_{0}$. Since $H_{0}$ is irreducible, we see that $H_{1}=H_{0}$.

(b) We apply [2, Vol. II, Exemple IV, pp. 144-145; Théorème 11, p. 171]. $H$ is the intersection of the algebraic group $G$-whose Lie algebra is $\mathfrak{D}$, since the characteristic is zero-and the algebraic group $\left\{\eta \in \operatorname{Hom}_{\Phi}(\Im, \Im) \mid \eta\right.$ is nonsingular and $\left.\mathfrak{\Im}_{1} \eta=\Im_{1}\right\}$-whose Lie algebra is $\left\{E \in \operatorname{Hom}_{\Phi}(\mathfrak{\Im}, \mathfrak{\Im}) \mid \mathfrak{\Im}_{1} E \subseteq \mathfrak{\Im}_{1}\right\}$. Thus the Lie algebra of $H$, which is the same as that of $H_{0}$, is the intersection of these two algebras, i.e. is $\left\{E \in \mathfrak{D} \mid \Im_{1} E \subseteq \mathfrak{\Im}_{1}\right\}$. But this is equal to $\mathfrak{D}_{0}$. For since $\mathfrak{\Im}_{1}$ is a sum of fields, every derivation which stabilizes $\mathfrak{\Im}_{1}$ annihilates it. Indeed, if $E \in \mathfrak{D}$, $\Im_{1} E \subseteq \Im_{1}$, then $e_{i} D=e_{i}^{2} D=2 e_{i} . e_{i} D(i=1, \ldots, k)$; hence $e_{i} D \in e_{i} . \Im_{1}=\Phi e_{i}$ and thus $e_{i} D=2 e_{i} D=0$.

Let $K=\left\{\tau^{-1} \eta \zeta \tau \mid \tau \in G_{0}, \zeta \in H_{0}\right\}$. Clearly $K \subseteq \eta G_{0}$.

Proposition 31. $K$ contains a nonempty open subset of $\eta G_{0}$.

Proof. First we note that $\eta H_{0}$ is épais (i.e. is irreducible and contains a relatively open subset of its closure). For it is the image of $H_{0}$, an épais set, under a polynomial map; here we are using [2, Vol. III, Proposition 20, p. 186; Lemme 3, p. 193]. We apply also the fact that every irreducible algebraic group is épais, and that $\Phi$ is algebraically closed. 
We consider the adjoint representation $\tau \mapsto$ ad $\tau$ of $G_{0}$ acting on $\operatorname{Hom}_{\Phi}(\Im, \Im):$ $x$ ad $\tau=\tau^{-1} x \tau$ for $x \in \operatorname{Hom}_{\Phi}(\Im, \Im)$. The image ad $G_{0}$ of $G_{0}$ under this rational representation is an algebraic group, hence an épais group of transformations of $\operatorname{Hom}_{\Phi}(\Im, \Im)$. Hence $K=\left(\eta H_{0}\right)$ ad $G_{0}$ is an épais set (it is the orbit of an épais set under an épais group). To prove the proposition we therefore need only show that $K$ is dense in $\eta G_{0}$.

If $x \in \eta H_{0}$, then by [2, Vol. III, Proposition 2, p. 192], $\mathfrak{I}(K, x) \supseteq x \mathfrak{Q}\left(\operatorname{ad} G_{0}\right)+$ $\mathfrak{I}\left(\eta H_{0}, x\right)$. Here $\mathfrak{T}(K, x)$ is the tangent space to $K$ at $x$ and $\mathfrak{L}\left(\operatorname{ad} G_{0}\right)$ is the Lie algebra of ad $G_{0}$. By [2, Vol. II, Proposition 6, p. 141], $\mathfrak{L}\left(\mathrm{ad} G_{0}\right)$ is ad $\mathfrak{D}$, the set of all mappings $A \rightarrow[A, D]$ of $\operatorname{Hom}_{\Phi}(\mathfrak{\Im}, \Im)$ into itself, for all $D \in \mathfrak{D}$. Thus we see that

$$
\begin{aligned}
\mathfrak{T}(K, x) & \supseteq[x, \mathfrak{D}]+\mathfrak{T}\left(\eta H_{0}, x\right)=[x, \mathfrak{D}]+\mathfrak{T}\left(x H_{0}, x\right) \\
& =[x, \mathfrak{D}]+x \mathfrak{T}\left(H_{0}, 1\right) \quad \text { by [2, Vol. III, p. 191] } \\
& =[x, \mathfrak{D}]+x \mathfrak{L}\left(H_{0}\right)=[x, \mathfrak{D}]+x \mathfrak{D}_{0} .
\end{aligned}
$$

This holds for all $x \in \eta H_{0}$.

The proof of the Corollaire on p. 192 of [2, Vol. III] implies that there is a nonempty Zariski open subset $E^{\prime}$ of $\eta H_{0}$ such that every point of $E^{\prime}$ is simple in $K$ (recall that this means that the dimension of the tangent space to $K$ at a point of $E^{\prime}$ is minimal and is the dimension of $K$ as an algebraic set). If $\zeta \in H_{0}$ then $\zeta$ fixes $\Im_{1}$ pointwise so $\eta \zeta$ fixes $\Im_{1}$ pointwise (here we use Propositions 28 and $30(\mathrm{a})$ ). Hence $\mathfrak{R} \zeta \subseteq \Re$, as before. Let $E^{\prime \prime}=\left\{x \in \eta H_{0} \mid \Im_{1}(x)=\Im_{1}(\eta)\right\}$. Now $E^{\prime \prime} \neq \varnothing$ since $\eta \in E^{\prime \prime}$; and $E^{\prime \prime}$ is open in $\eta H_{0}$, for $E^{\prime \prime}=\left\{x \in \eta H_{0} \mid \operatorname{det}[(1-x) \mid \mathfrak{N}] \neq 0\right\}$. So $E^{\prime} \cap E^{\prime \prime} \neq \varnothing$. Let $\eta^{\prime}$ belong to $E^{\prime} \cap E^{\prime \prime}$. Then

$$
\begin{aligned}
\mathfrak{T}\left(K, \eta^{\prime}\right) \supseteq\left[\eta^{\prime}, \mathfrak{D}\right]+\eta^{\prime} \mathfrak{D}_{0} & =\left\{\eta^{\prime} D-D \eta^{\prime} \mid D \in \mathfrak{D}\right\}+\eta^{\prime} \mathfrak{D}_{0} \\
& =\eta^{\prime}\left[\left\{D-\eta^{\prime-1} D \eta^{\prime} \mid D \in \mathfrak{D}\right\}+\mathscr{D}_{0}\right] .
\end{aligned}
$$

Now $\eta^{\prime}$ has $\Im_{1}$ as its 1-eigenspace. So applying Proposition 29 to $\eta^{\prime}$ rather than $\eta$, we find that $\left\{D-\eta^{\prime-1} D \eta^{\prime} \mid D \in \mathfrak{D}\right\}+\mathfrak{D}_{0}=\mathfrak{D}$. I.e. $\mathfrak{T}\left(K, \eta^{\prime}\right) \supseteq \eta^{\prime} \mathfrak{D}$. Thus $\operatorname{dim} K=$ $\operatorname{dim} \eta^{\prime} \mathfrak{D}=\operatorname{dim} \mathfrak{D}=\operatorname{dim} \eta G_{0}$, since $\eta^{\prime}$ is simple in $K$. Since $K \subseteq \eta G_{0}$, this means that $K$ is dense in $\eta G_{0}$.

THEOREM 6. If $\eta \in G$ is such that $\mathfrak{\Im}_{1}(\eta)$ is associative, then $\eta$ is regular.

Proof. Since the set of regular elements of $\eta G_{0}$ is open, its intersection with $K$ (defined above) is nonempty. Let $\tau^{-1} \eta \zeta \tau \in K$ be regular. Then $\eta \zeta$ is regular, since $\Im_{1}(\eta \zeta)=\Im_{1}\left(\tau^{-1} \eta \zeta \tau\right) \tau^{-1}$. But since $\eta \zeta$ fixes $\Im_{1}$, the fixed-point space of $\eta, \eta$ too must be regular.

We note that $\lambda I$, scalar multiplication in $\mathfrak{\Im}$ by $0 \neq \lambda$, is an element of $\Gamma$ and that $(\lambda I)^{\#}=\lambda I$. For $(\lambda I) U_{a}(\lambda I)=\lambda^{2} U_{a}=U_{\lambda a}=U_{a(\lambda I)}$. Also $\lambda \mapsto \lambda I$ is a polynomial map from $\Phi-\{0\}$, an irreducible set, to $\Gamma$. The image of this map contains $I$, which implies that $\lambda I \in \Gamma_{0}$. For brevity we write $\tilde{\lambda}$ for $(\lambda I)^{\sim}$. Thus $\tilde{\lambda} \in$ Aut $_{0} \Omega$ and if $a, b \in \mathfrak{s}, L \in \mathfrak{L}$ then $\tilde{\lambda}: a+\bar{b}+L \rightarrow \lambda a+\lambda^{-1} \bar{b}+L$. 
We can now prove the following principal result:

Theorem 7. Let $G_{i}$ be a component of $G$. Let $N$ be the index of $G_{i}, M$ the index of the component of Aut $\AA$ to which $\tilde{G}_{i}$ belongs, and $P$ the index of the component of Aut $\mathfrak{D}^{\prime}$ to which $\breve{G}_{i}$ belongs. Since for $\zeta \in G_{i}, \tilde{\zeta} \mid \Subset$ is independent of $\zeta$ (Proposition $5)$, we can let $Q$ be the dimension of $\mathfrak{E}_{1}\left(\tilde{\zeta} \mid \mathfrak{S}_{)}\right.$, the 1-eigenspace of this well-defined action on $\mathbb{E}$.

Then $N=M-P-Q$.

Proof. We first choose $\eta \in G_{i}$ so that $\eta$ is regular and $\check{\eta}$ is regular (as an element of Aut $\left.\mathfrak{D}^{\prime}\right)$. That this is possible we see as follows. We have seen that the set of regular elements of $G_{i}$ is a nonempty open subset of $G_{i}$. We show that $\left\{\eta \in G_{i} \mid \check{\eta}\right.$ is regular $\}$ is also nonempty and open. Since $G_{i}$ is irreducible, these two open sets

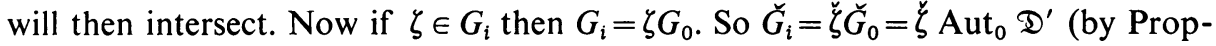
osition 5). Thus $\breve{G}_{i}$ is an entire component of Aut $\mathfrak{D}^{\prime}$. The set of regular elements of $\breve{G}_{i}$ is thus nonempty and open in $\breve{G}_{i}$. Since $\eta \mapsto \check{\eta}$ is a rational map and hence open [2, Vol. III, Proposition 6a, p. 176], $\left\{\eta \in G_{i} \mid \check{\eta}\right.$ is regular $\}$ is open in $G_{i}$.

Choose nonzero $\lambda \in \Phi$ so that neither $\lambda$ nor $\lambda^{-1}$ is an eigenvalue of $\eta$. Then $\tilde{\eta} \tilde{\lambda}$ is in the same component of Aut $\mathfrak{\Omega}$ as $\tilde{\eta}$. We find the 1-eigenspace $\mathfrak{R}_{1}=\Re_{1}(\tilde{\eta} \tilde{\lambda})$.

Since $\tilde{\eta} \tilde{\lambda} \in \tilde{\Gamma}, \tilde{\eta} \tilde{\lambda}$ stabilizes $\mathfrak{\Im}, \bar{\Im}$, and $\mathfrak{L}$. So $\mathfrak{R}_{1}=\Im_{1}\left(\tilde{\eta} \tilde{\lambda} \mid \Im_{1}\right) \oplus \mathfrak{\Im}_{1}(\tilde{\eta} \tilde{\lambda} \mid \bar{\Im}) \oplus \mathfrak{Q}_{1}(\tilde{\eta} \tilde{\lambda} \mid \mathfrak{L})$. Now $\tilde{\eta} \tilde{\lambda} \mid \mathfrak{\Im}$ and $\tilde{\eta} \tilde{\lambda} \mid \bar{\Im}$ do not have 1 as an eigenvalue: for if $a \in \mathfrak{\Im}$ is such that $a \tilde{\eta} \tilde{\lambda}=a$, then $\lambda a \eta=a$, i.e. $a \eta=\lambda^{-1} a$, which is impossible by the choice of $\lambda$. Similarly, if $\bar{a} \tilde{\eta} \tilde{\lambda}=\bar{a}$, then $\lambda^{-1} a \eta=a$, which means that $a \eta=\lambda a$, again an impossibility. We conclude that $\mathfrak{\Omega}_{1} \subseteq \mathfrak{L}$. Now if $R_{a}+E+D$ is an arbitrary element of $\mathfrak{L}$ $\left(a \in \mathfrak{\Im}, E \in \mathfrak{E}, D \in \mathfrak{D}^{\prime}\right)$ then $\left(R_{a}+E+D\right) \tilde{\eta} \tilde{\lambda}=R_{a \eta}+E \tilde{\eta}+D \tilde{\eta}$, where $E \tilde{\eta} \in \mathfrak{E}$ and $D \tilde{\eta} \in \mathfrak{D}^{\prime}$. Hence $\mathfrak{R}_{1}=R_{\mathfrak{I}_{1}(\eta)}+\mathfrak{E}_{1}(\tilde{\eta} \mid \mathfrak{E})+\mathfrak{D}_{1}^{\prime}(\check{\eta})$.

We claim that $\mathfrak{R}_{1}$ is abelian. $\mathfrak{D}_{1}^{\prime}(\check{\eta})$ is abelian by the results on Lie algebras cited in the Introduction and the fact that $\check{\eta}$ is regular. Since $\mathbb{E}_{1}(\tilde{\eta} \mid \mathfrak{E})$ is contained in the center of $\mathfrak{D}, \mathfrak{S}_{1}(\tilde{\eta} \mid \mathfrak{E})+\mathfrak{D}_{1}^{\prime}(\check{\eta})$ is abelian. $\mathfrak{\Im}_{1}(\eta)$ is a direct sum of fields, say $\Im_{1}(\eta)=$ $\Phi e_{1} \oplus \cdots \oplus \Phi e_{k}$, where $e_{1}, \ldots, e_{k}$ are orthogonal idempotents (here we use Theorem 5 and the fact that $\eta$ is regular). Thus $\left[R_{e_{i}}, R_{e_{j}}\right]=0$ for all $i, j$; this is always true of orthogonal idempotents and follows from properties of the Peirce decomposition. Hence $\left[R_{\mathfrak{I}_{1}(\eta)}, R_{\mathfrak{I}_{1}(\eta)}\right]=0$. Finally, if $D \in \mathfrak{E}_{1}(\tilde{\eta} \mid \mathfrak{E})+\mathfrak{D}_{1}^{\prime}(\check{\eta})$, then the fact that $\mathfrak{\Re}_{1}$ is a subalgebra implies that $R_{\mathfrak{I}_{1}(\eta) D}=\left[R_{\mathfrak{I}_{1}(\eta)}, D\right] \subseteq R \mathfrak{I}_{1}(\eta)$, i.e. $\mathfrak{I}_{1}(\eta) D$ $\subseteq \Im_{1}(\eta)$. Thus, as in the proof of Proposition 30(b), $\mathfrak{\Im}_{1}(\eta) D=0$. This means that $\left[R_{\mathfrak{I}_{1}(\eta)}, \mathfrak{E}_{1}(\tilde{\eta} \mid \mathfrak{E})+\mathfrak{D}_{1}^{\prime}(\tilde{\eta})\right]=0$.

Since $\Omega_{1}$ is abelian, $\tilde{\eta} \tilde{\lambda}$ is regular, again by the results on Lie algebras cited in the Introduction. Hence $\operatorname{dim} \Re_{1}=M$. Certainly $\operatorname{dim} \Im_{1}(\eta)=N, \operatorname{dim} \mathfrak{\aleph}_{1}(\tilde{\eta} \mid \Subset)=Q$, and $\operatorname{dim} \mathfrak{D}_{1}^{\prime}(\grave{\eta})=P$. This means that $N=M-P-Q$.

Corollary. The index of $G_{0}$ is rank $\mathfrak{\AA}$ - rank $\mathfrak{D}$.

Proof. The results on Lie algebras cited in the Introduction imply that if $\mathfrak{B}$ is a semisimple Lie algebra over $\Phi$, then the index of $\operatorname{Aut}_{0} \mathfrak{B}$ is rank $\mathfrak{B}$, i.e. the dimension of a Cartan subalgebra of $\mathfrak{B}$. If $\zeta \in G_{0}$ then $\tilde{\zeta}$ fixes $\&$ pointwise and hence 
$\mathfrak{E}_{1}(\tilde{\zeta} \mid \mathfrak{F})=\mathfrak{F}$. Also $\check{G}_{0}=$ Aut $_{0} \mathfrak{D}^{\prime}$ and $\tilde{G}_{0}$ belongs to Aut $\mathfrak{R}$. Thus in the notation of Theorem $7, M=\operatorname{rank} \Re, P=\operatorname{rank} \mathfrak{D}^{\prime}$, and $Q=\operatorname{dim} \mathfrak{E}$. Since $P+Q=\operatorname{rank} \mathfrak{D}$, by the Lemma preceding Proposition 1, we are done.

We note that if $G_{i}$ is a component of $G$, then every element of $G_{i}$ fixes a subspace of dimension at least index $\left(G_{i}\right)$. The proof of this uses the fact that a regular automorphism $\eta$ fixes $\Im_{1}(\eta)$ pointwise (Proposition 28 ), and is otherwise the same as in the case of Lie algebras [9, Theorem 10, p. 314]. The above Corollary now has the following infinitesimal analogue.

THEOREM 8. Let $A$ be the minimum dimension of the kernel of a derivation of $\Im$ and $B$ the minimum (for all $D \in \mathfrak{D}$ ) of $\operatorname{dim} \Im_{0}(D)$, the 0-eigenspace of $D$. Then $A=B=$ rank $\mathfrak{R}-$ rank $\mathfrak{D}$.

Proof. We first show that $\mathfrak{D}$ contains elements which are regular in $\mathfrak{L}$, i.e. there exists $D \in \mathfrak{D}$ such that $\mathfrak{L}_{0}\left(\operatorname{ad}_{\mathfrak{L}} D\right)$ is a Cartan subalgebra of $\mathfrak{L}$. As before $\mathfrak{L}=R_{\mathfrak{U}}+$ $\mathfrak{W}+\sum_{\alpha} \mathfrak{L}_{\alpha}$, where the summation is over all roots of $\mathfrak{L}^{\prime}$. Since for any root $\alpha$ of $\mathfrak{L}^{\prime}, \alpha(\mathfrak{B}) \neq 0$ (by Proposition 15 ), we can choose $D \in \mathfrak{W}$ to be a nonzero of all the roots of $\mathfrak{R}^{\prime}$. If $a \in \mathfrak{A}, E \in \mathfrak{W}, L_{\alpha} \in \mathfrak{L}_{\alpha}$, then $L=R_{a}+E+\sum_{\alpha} L_{\alpha}$ is in $\mathfrak{Q}_{0}\left(\operatorname{ad}_{\mathfrak{L}} D\right)$ if and only if for some $n, L(\operatorname{ad} D)^{n}=0$. But if $n \geqq 1$ then $L(\operatorname{ad} D)^{n}=\sum_{\alpha} \alpha(D)^{n} L_{\alpha}$ which is zero only if all $L_{\alpha}$ are zero. We conclude that $\mathfrak{S}=\mathfrak{L}_{0}\left(\operatorname{ad}_{\mathfrak{L}} D\right)$, and thus that $D$ is regular in $\mathfrak{R}$.

We now consider the following four sets of derivations:

(i) The set of $D$ such that $D$ is regular-i.e., such that $\mathfrak{D}_{0}\left(\operatorname{ad}_{\mathfrak{D}} D\right)$ is a Cartan subalgebra of $\mathfrak{D}$.

(ii) The set of $D$ such that $D$ is regular in $\mathfrak{L}$.

(iii) The set of $D$ such that dim kerrel $D=A$.

(iv) The set of $D$ such that $\operatorname{dim} \Im_{0}(D)=B$.

Each of these sets contains a nonempty Zariski open subset of $\mathfrak{D}$. This is well known for the first [8, p. 61] and has been shown for the second: the second set is nonempty and is the intersection of an open set of $\mathfrak{L}$ with $\mathfrak{D}$, hence is open in $\mathfrak{D}$. To see that the third set contains an open set, note that the dimension of the kernel of $D$ is $\operatorname{dim} \Im-$ rank $D$, so that the third set is the set of derivations of maximal rank. If we choose a basis for $\mathfrak{\Im}$ and regard $\operatorname{Hom}_{\Phi}(\mathfrak{T}, \Im)$ as a complete matrix algebra, we see that the largest $k$ such that there is a $k \times k$ minor which is nonzero on $\mathfrak{D}$ is this maximal rank. The third set contains the nonzeroes of such a minor, an open set. To see that the fourth set is open, let det $(\lambda I-x)=\lambda^{n}-\sigma_{1}(x) \lambda^{n-1}+$ $\cdots+(-1)^{n} \sigma_{n}(x)$ for $x$ a generic element of $\operatorname{Hom}_{\Phi}(\Im, \Im)$ (see [10, p. 222] for the definition of generic elements). Here $\sigma_{1}, \ldots, \sigma_{n}$ are polynomial functions on $\operatorname{Hom}_{\Phi}(\Im, \Im)$, hence on $\mathfrak{D}$. If $\sigma_{n}, \sigma_{n-1}, \ldots, \sigma_{n-k+1}$ are all identically zero on $\mathfrak{D}$ but $\sigma_{n-k}$ is not, then $\operatorname{dim} \Im_{0}(D)=B$ if and only if $\sigma_{n-k}(D) \neq 0$.

Thus we can choose a derivation $D$ which lies in all four of the above sets. Since for $a \in \Im, E \in \mathfrak{D},\left(R_{a}+E\right)(\operatorname{ad} D)^{n}=R_{a D^{n}}+E(\operatorname{ad} D)^{n}$, it follows that $\mathfrak{Q}_{0}\left(\operatorname{ad}_{\mathfrak{L}} D\right)$ $=R_{\mathfrak{I}_{0}(D)}+\mathfrak{D}_{0}\left(\operatorname{ad}_{\mathfrak{D}} D\right)$. That $D$ is in the first and second sets shows that $\mathfrak{D}_{0}\left(\operatorname{ad}_{\mathfrak{D}} D\right)$ 
is a Cartan subalgebra of $\mathfrak{D}$ and $\mathfrak{L}_{0}\left(\operatorname{ad}_{\mathfrak{L}} D\right)$ is a Cartan subalgebra of $\mathfrak{L}$. Since Cartan subalgebras of $\mathfrak{L}$ are abelian (recall that $\mathfrak{L}$ is completely reducible), $\left[R_{\mathfrak{I}_{0}(D)}, \mathfrak{D}_{0}\left(\operatorname{ad}_{\mathfrak{D}} D\right)\right]=0$. In particular, $D \in \mathfrak{D}_{0}\left(\operatorname{ad}_{\mathfrak{D}} D\right)$, so $\mathfrak{\Im}_{0}(D) D=0$. Thus $\mathfrak{\Im}_{0}(D)$ is the kernel of $D$ and $A=B$. Finally, we note that $\operatorname{dim} \Im_{0}(D)=\operatorname{dim} \mathfrak{\Omega}_{0}(\operatorname{ad} D)$ $-\operatorname{dim} \mathfrak{D}_{0}(\operatorname{ad} D)=\operatorname{rank} \mathfrak{L}-\operatorname{rank} \mathfrak{D}=\operatorname{rank} \mathfrak{R}-\operatorname{rank} \mathfrak{D}$.

5. Examples. Our purpose in this section is to apply the preceding results to the simple Jordan algebras over $\Phi$. We first make some general remarks. We recall that if $\mathfrak{F}$ is simple then $\mathfrak{C}$ is one-dimensional and that $\mathfrak{E}=0$ unless $\mathfrak{\Im}$ is threedimensional. Also, if each of the idempotents $e_{\rho}, \rho \in P$, is primitive then every root of $\Sigma_{\varepsilon}$ is of the form $\bar{\omega}, \omega$ a root of $\mathfrak{D}^{\prime}$. To see this, suppose that $\alpha$ is a root of $\mathfrak{R}^{\prime}$ such that $\alpha_{+}$is not $\bar{\omega}$ for any root $\omega$ of $\mathfrak{D}^{\prime}$. Proposition 17 implies that a root vector for $\alpha$ has the form $R_{x}, x \in \mathfrak{\Im}$. Since $\left[R_{x}, R_{\mathfrak{U}}\right] \subseteq \mathfrak{D} \cap \Phi R_{x}$, clearly $\left[R_{x}, R_{\mathfrak{U}}\right]=0$. If $\rho \in P$, let $x=x_{1}+x_{1 / 2}+x_{0}$, where $x_{i} \in \Im_{i}\left(e_{\rho}\right)$, the $i$-Peirce space of the idempotent $e_{\rho}$. Then $e_{\rho}\left[R_{x}, R_{e_{\rho}}\right]=0$, so that $\left(x \cdot e_{\rho}\right) \cdot e_{\rho}=x \cdot e_{\rho}$; this implies that $x_{1}+\frac{1}{4} x_{1 / 2}=x_{1}+$ $\frac{1}{2} x_{1 / 2}$, which means that $x_{1 / 2}=0$. We conclude that if $\mathfrak{\Im}=\oplus \sum \mathfrak{\Im}_{\rho \sigma}$ (where the sum is over unordered pairs of roots $\rho, \sigma \in P$ ) is the Peirce decomposition of $\Im$ with respect to the idempotents $e_{\rho}, \rho \in P$, then for each $\rho \in P, x \in \Im_{0}\left(e_{\rho}\right)+\Im_{1}\left(e_{\rho}\right)$, i.e. that $x \in \oplus \sum_{\rho \in P} \mathfrak{\Im}_{\rho \rho}$. Since the $e_{\rho}$ 's are primitive, $\mathfrak{\Im}_{\rho \rho}=\Phi e_{\rho}$. Hence $x \in \sum_{\rho \in P} \Phi e_{\rho}=$ A. I.e. $R_{x} \in R_{\mathfrak{A}} \subseteq \mathfrak{S}$, a contradiction to the hypothesis that $R_{x}$ is a root vector of $\mathfrak{R}^{\prime}$. Thus if all $e_{\rho}$ are primitive the proof of Proposition $25(\mathrm{~b})$ shows that $\left(W\left(\mathfrak{D}^{\prime}\right)\right)^{-}=$ $W\left(\Sigma_{\varepsilon}\right)$; in this case $\Gamma_{0} \cap G=G_{0}$, by Theorem 4 .

We now work our way through the classification of simple algebras (see [10, Chapters 3-5] for details of the classification theory).

(I) $\mathfrak{I}$ is three-dimensional, i.e. $\mathfrak{J}=\Phi 1 \oplus \mathfrak{B}$, where $\mathfrak{B}$ is a two-dimensional vector space with a nondegenerate symmetric bilinear form (, ). We choose a basis $\left\{v_{1}, v_{2}\right\}$ of $\mathfrak{B}$ such that $\left(v_{1}, v_{2}\right)=1$ and $\left(v_{1}, v_{1}\right)=\left(v_{2}, v_{2}\right)=0$. Then with respect to the basis $\left\{1, v_{1}, v_{2}\right\}$ of $\mathfrak{I}, \mathfrak{D}=\mathfrak{W}$ consists of all scalar multiples of the matrix

$$
E=\left(\begin{array}{rrr}
0 & 0 & 0 \\
0 & 1 & 0 \\
0 & 0 & -1
\end{array}\right) .
$$

The kernel of this transformation is $\Phi 1$, so that $\mathfrak{U}=\Phi 1$ and

$$
\mathfrak{S}=\left\{\psi R_{1}+\omega E \mid \psi, \omega \in \Phi\right\} .
$$

By abuse of notation, we let $\{\psi, \omega\}$ be the basis of $\mathfrak{S}^{*}$ dual to the basis $\left\{R_{1}, E\right\}$ of $\mathfrak{S}$. We exhibit the root spaces of $\mathfrak{R}$ : if $H=\psi R_{1}+\omega E$, then

$$
\begin{aligned}
{[1, H] } & =\psi 1, & {[\overline{1}, H] } & =-\psi \overline{1}, \\
{\left[v_{1}, H\right] } & =(\psi+\omega) v_{1}, & {\left[\bar{v}_{1}, H\right] } & =(-\psi+\omega) \bar{v}_{1}, \\
{\left[v_{2}, H\right] } & =(\psi-\omega) v_{2}, & {\left[\bar{v}_{2}, H\right] } & =(-\psi-\omega) \bar{v}_{2}, \\
{\left[R_{v_{1}}, H\right] } & =\omega R_{v_{1} E}=\omega R_{v_{1}}, & {\left[R_{v_{2}}, H\right] } & =-\omega R_{v_{2}} .
\end{aligned}
$$


Thus the roots of $\Re$ are $\pm \psi, \pm \psi \pm \omega, \pm \omega$. Comparison with [8, p. 139] shows that the root space of $\mathfrak{\Re}$ is of type $B_{2}$. Now $\varepsilon^{*}$ maps $\psi \rightarrow-\psi$ and $\omega \rightarrow \omega$. $\mathfrak{B}=\mathfrak{S}_{1}$, so that $\mathfrak{S}_{1}^{*}=\mathfrak{W B}^{*}=\mathfrak{E}^{*}=\Phi \omega$ (identifying $\mathfrak{S}_{1}^{*}$ with $\left(\mathfrak{S}_{1}^{*}\right)^{\wedge}$, as we shall in all these examples). Thus $\{\omega\}$ is a simple system of roots of $\mathfrak{Q}^{\prime}$ stabilized by $\varepsilon^{*}$. Since $(\psi-\omega)\left(R_{1}\right)$ $=1,\{\psi-\omega, \omega\}$ is the corresponding simple system of roots of $\mathfrak{\AA}$. I.e. in the notation we have consistently used, $\rho_{1}=\psi-\omega$ and $\hat{\alpha}_{1}=\omega$. The Dynkin diagram of $\AA$ (with $\rho_{1}$ circled) is

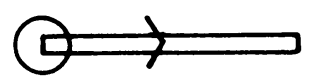

The number of automorphisms of this diagram is 1 -i.e. $\Gamma=\Gamma_{0} \cdot \mathscr{D}^{\prime}=0$, so $\left(W\left(\mathfrak{D}^{\prime}\right)\right)^{-}=\langle 1\rangle$. Since $\Sigma_{\varepsilon}$ is of type $A_{1}, W\left(\Sigma_{\varepsilon}\right)$ has order 2 . Thus $G$ has two components. The component $G_{0}$ of the identity fixes $E$. The other component, $G_{1}$, corresponding in the root space $\Sigma_{\varepsilon}$ to the symmetry in the direction of $\omega$, maps $E$ to $-E$. Thus the index of $G_{0}$ is $2-1=1$, whereas the index of $G_{1}$ is $2-0=2$.

In the remaining examples, $\mathfrak{E}=0$. So $\mathfrak{W}=\mathfrak{B}_{1}$ and we can identify $\mathfrak{B}_{1}^{*}$ and $\mathfrak{B S}^{*}$. We also identify $\mathfrak{H}_{1}^{*}$ and $\left(\mathfrak{H}_{1}^{*}\right)^{\wedge}$, as above.

(II) $\mathfrak{\Im}=\Phi 1 \oplus \mathfrak{B}$, where $\operatorname{dim} \mathfrak{B}=n=2 l, l \geqq 2$. Here $\mathfrak{D}$ consists of linear transformations of $\mathfrak{\Im}$ which map $1 \rightarrow 0$ and are skew transformations on $\mathfrak{B}$. We choose a basis $u_{1}, \ldots, u_{i}, v_{1}, \ldots, v_{l}$ of $\mathfrak{B}$ with respect to which the form on $\mathfrak{B}$ has matrix $\left(\begin{array}{ll}0 & I_{l} \\ l_{l} & 0\end{array}\right)$; here $I_{l}$ is an $l \times l$ identity matrix. We thus have the basis $1, u_{1}, \ldots, u_{l}$, $v_{1}, \ldots, v_{l}$ of $\Im$. If $a, b$ are elements of this basis, by $E_{a, b}$ we mean the corresponding matrix unit: the linear transformation of $\Im$ which maps $a$ to $b$ and all basis elements other than $a$ to 0 . Then if $H_{i}=E_{u_{i}, u_{i}}-E_{v_{i}, v_{i}}$, a Cartan subalgebra $\mathfrak{W}$ of $\mathfrak{D}$ is $\left\{\sum_{i=1}^{l} \omega_{i} H_{i} \mid \omega_{i} \in \Phi\right\} . \mathscr{A}$ again is $\Phi 1$, so that $\mathfrak{S}=\left\{\psi R_{1}+\sum_{i} \omega_{i} H_{i} \mid \psi, \omega_{i} \in \Phi\right.$ for all $\left.i\right\}$; as before we denote by $\psi, \omega_{1}, \ldots, \omega_{l}$ the corresponding dual basis of $\mathfrak{S}^{*}$. We exhibit the root spaces of $\Re$ : if $H=\psi R_{1}+\sum_{i} \omega_{i} H_{i}$, then

$$
\begin{aligned}
& {[1, H]=\psi 1, \quad[\overline{1}, H]=-\psi \overline{1},} \\
& {\left[u_{i}, H\right]=\left(\psi+\omega_{i}\right) u_{i}, \quad\left[\bar{u}_{i}, H\right]=\left(-\psi+\omega_{i}\right) \bar{u}_{i},} \\
& {\left[v_{i}, H\right]=\left(\psi-\omega_{i}\right) v_{i}, \quad\left[\bar{v}_{i}, H\right]=\left(-\psi-\omega_{i}\right) \bar{v}_{i} \text {. }}
\end{aligned}
$$

A basis for $\mathfrak{D}$ consists of the transformations $H_{i}, E_{u_{j}, u_{i}}-E_{v_{i}, v_{j}}(i \neq j), E_{v_{i}, u_{j}}-E_{v_{j}, u_{i}}$ $(i<j), E_{u j, v_{i}}-E_{u_{i}, v_{j}}(i<j)$. By [7, p. 141], we see that

$$
\begin{array}{ll}
{\left[E_{u_{j}, u_{i}}-E_{v_{i}, v_{j}}, H\right]=\left(\omega_{i}-\omega_{j}\right)\left(E_{u_{j}, u_{i}}-E_{v_{i}, v_{j}}\right)} & (i \neq j), \\
{\left[E_{v_{i}, u_{j}}-E_{v_{j}, u_{i}}, H\right]=\left(\omega_{i}+\omega_{j}\right)\left(E_{v_{i}, u_{j}}-E_{v_{j}, u_{i}}\right)} & (i<j), \\
{\left[E_{u_{j}, v_{i}}-E_{u_{i}, v_{j}}, H\right]=\left(-\omega_{i}-\omega_{j}\right)\left(E_{u_{j}, v_{i}}-E_{u_{i}, v_{j}}\right)} & (i<j) .
\end{array}
$$

In addition

$$
\left[R_{u_{i}}, H\right]=\omega_{i} R_{u_{i}}, \quad\left[R_{v_{i}}, H\right]=-\omega_{i} R_{v_{i}} .
$$

Since $\mathfrak{A}^{\prime}=0, \mathfrak{W}^{*}=\mathfrak{H}_{1}^{*}$. Thus all roots of $\mathfrak{Q}^{\prime}$ are fixed by $\varepsilon^{*}$. The roots of $\mathfrak{L}^{\prime}$ which are roots of $\mathfrak{D}^{\prime}$ are $\omega_{i}-\omega_{j}(i \neq j)$ and $\pm\left(\omega_{i}+\omega_{j}\right)(i<j)$. The additional roots of $\mathfrak{L}^{\prime}$ are 
$\pm \omega_{i}$. A simple system of roots of $\mathfrak{R}^{\prime}$ is $\omega_{1}-\omega_{2}, \omega_{2}-\omega_{3}, \ldots, \omega_{l-1}-\omega_{l}, \omega_{l}$ and thus $\mathfrak{L}^{\prime}$ is of type $B_{l}$. The simple system of roots of $\mathfrak{D}$ consisting of positive roots (see the discussion preceding Proposition 26) is $\omega_{1}-\omega_{2}, \omega_{2}-\omega_{3}, \ldots, \omega_{l-1}-\omega_{l}, \omega_{l-1}+$ $\omega_{l}$ (this last root is positive since $\left.\omega_{l-1}+\omega_{l}=\left(\omega_{l-1}-\omega_{l}\right)+2 \omega_{l}\right)$. So $\mathfrak{D}^{\prime}$ is of type $D_{l}$. $W\left(\Sigma_{\varepsilon}\right)$ is generated by the symmetries in the directions of the simple roots. The first $l-1$ simple roots of $\Sigma_{\varepsilon}$ are roots of $\mathfrak{D}^{\prime}$. The symmetry $w_{\omega_{l}}$ fixes the roots $\omega_{1}-\omega_{2}, \ldots, \omega_{l-2}-\omega_{l-1}$ and interchanges the roots $\omega_{l-1}-\omega_{l}$ and $\omega_{l-1}+\omega_{l}$. Thus the index of $W\left(\mathfrak{D}^{\prime}\right)$ in $W\left(\Sigma_{\varepsilon}\right)=W\left(\mathfrak{R}^{\prime}\right)$ is 2 ; the two cosets of $W\left(\mathfrak{D}^{\prime}\right)$ are $W\left(\mathfrak{D}^{\prime}\right)$ and $w_{\omega_{l}} W\left(\mathfrak{D}^{\prime}\right)$.

The roots of $\AA$ are $\pm \psi, \pm \omega_{i}, \omega_{i}-\omega_{j}(i \neq j), \pm\left(\omega_{i}+\omega_{j}\right)(i<j)$, and $\pm\left(\psi \pm \omega_{i}\right)$. Thus $\mathfrak{R}$ is of type $B_{l+1}$. The simple system of roots of $\mathfrak{\Re}$ corresponding to the above simple system of roots of $\mathfrak{R}^{\prime}$ is $\psi-\omega_{1}, \omega_{1}-\omega_{2}, \ldots, \omega_{l-1}-\omega_{l}, \omega_{l}$ : in our standard notation, $\rho_{1}=\psi-\omega_{1}$ and $\left\{\hat{\alpha}_{1}, \ldots, \hat{\alpha}_{l}\right\}=\left\{\omega_{1}-\omega_{2}, \ldots, \omega_{l}\right\}$. The Dynkin diagram of $\Re$ (with $\rho_{1}$ circled) is

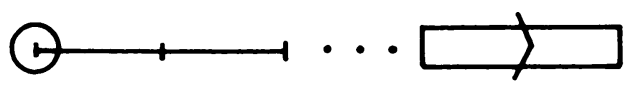

So $\Gamma=\Gamma_{0}$ and $G$ has two components. The index of $G_{0}$ is $(l+1)-l=1$. Since the reflection $w_{\omega_{l}}$ of the root space of $\mathfrak{L}^{\prime}$ has $l-1$ orbits in its action on the simple system of roots of $\mathfrak{R}^{\prime}$, the other component of $G$ has index $(l+1)-(l-1)=2$.

(III) $\mathfrak{\Im}=\Phi 1 \oplus \mathfrak{B}$, where $\operatorname{dim} \mathfrak{B}=n=2 l+1$. Again, $\mathfrak{D}$ consists of linear transformations of $\Im$ which map $1 \rightarrow 0$ and are skew transformations on $\mathfrak{B}$. We choose a basis $t, u_{1}, \ldots, u_{l}, v_{1}, \ldots, v_{l}$ of $\mathfrak{B}$ with respect to which the form on $\mathfrak{B}$ has matrix

$$
\left(\begin{array}{lll}
1 & 0 & 0 \\
0 & 0 & I_{l} \\
0 & I_{l} & 0
\end{array}\right)
$$

$\Im$ has the basis $\left\{1, t, u_{1}, \ldots, u_{l}, v_{1}, \ldots, v_{l}\right\} ; E_{a, b}$ is as in the preceding example. A Cartan subalgebra of $\mathfrak{W}$ is spanned by $H_{i}=E_{u_{i}, u_{i}}-E_{v_{i}, v_{i}}$. In this case $\mathfrak{A}=\Phi 1 \oplus$ $\Phi t=\Phi \frac{1}{2}(1+t) \oplus \Phi \frac{1}{2}(1-t)$; here $\frac{1}{2}(1+t)$ and $\frac{1}{2}(1-t)$ are primitive idempotents. We have seen that this means $(W(\mathfrak{D}))^{-}=W\left(\Sigma_{\varepsilon}\right)$.

We let $H_{0}=R_{t}$. Then a typical element of $\mathfrak{S}$ is $\psi R_{1}+\sum_{i=0}^{l} \omega_{i} H_{i}$. A basis for $\mathfrak{D}$ is $H_{1}, \ldots, H_{l}, E_{u_{j}, u_{i}}-E_{v_{i}, v_{j}}(i \neq j), E_{v_{i}, u_{j}}-E_{v_{j}, u_{i}}(i<j), E_{u_{j}, v_{i}}-E_{u_{i}, v},(i<j), E_{t, u_{i}}-$ $E_{v_{i}, t}$ (all $\left.i\right), E_{u_{i}, t}-E_{t, v_{i}}$ (all $i$ ). We exhibit the root spaces of $\mathfrak{R}:$ if $H=\psi R_{1}+$ $\sum_{i=0}^{l} \omega_{i} H_{i}$, then

$$
\begin{array}{rlrl}
{[1+t, H]} & =[1, H]+[t, H]=\left(\psi 1+\omega_{0} t\right)+\left(\psi t+\omega_{0} 1\right)=\left(\psi+\omega_{0}\right)(1+t), \\
{[\overline{1+t}, H]} & =\left(-\psi-\omega_{0}\right)(\overline{1+t}), & & \\
{[1-t, H]} & =\left(\psi-\omega_{0}\right)(1-t), & {[\overline{1-t}, H]} & =\left(-\psi+\omega_{0}\right)(\overline{1-t}), \\
{\left[u_{i}, H\right]} & =\left(\psi+\omega_{i}\right) u_{i}, & {\left[\bar{u}_{i}, H\right]} & =\left(-\psi+\omega_{i}\right) \bar{u}_{i}, \\
{\left[v_{i}, H\right]} & =\left(\psi-\omega_{i}\right) v_{i}, & {\left[\bar{v}_{i}, H\right]} & =\left(-\psi-\omega_{i}\right) \bar{v}_{i} .
\end{array}
$$


Using [8, p. 139], we see that

$$
\begin{array}{ll}
{\left[E_{u_{j}, u_{i}}-E_{v_{i}, v_{j}}, H\right]=\left(\omega_{i}-\omega_{j}\right)\left(E_{u_{j}, u_{i}}-E_{v_{i}, v_{j}}\right)} & (i \neq j, i, j \geqq 1), \\
{\left[E_{v_{i}, u_{j}}-E_{v_{j}, u_{i}}, H\right]=\left(\omega_{i}+\omega_{j}\right)\left(E_{v_{i}, u_{j}}-E_{v_{j}, u_{i}}\right)} & (1 \leqq i<j), \\
{\left[E_{u_{j}, v_{i}}-E_{u_{i}, v_{j}}, H\right]=\left(-\omega_{i}-\omega_{j}\right)\left(E_{u_{j}, v_{i}}-E_{u_{i}, v_{j}}\right)} & (1 \leqq i<j) .
\end{array}
$$

The same reference shows that $\left[E_{t, u_{i}}-E_{v_{i}, t}, \sum_{i=1}^{l} \omega_{i} H_{i}\right]=\omega_{i}\left(E_{t, u_{i}}-E_{v_{i}, t}\right)$ and $\left[E_{u_{i}, t}-E_{t, v_{i}}, \sum_{i=1}^{l} \omega_{i} H_{i}\right]=-\omega_{i}\left(E_{u_{i}, t}-E_{t, v_{i}}\right)$. Since $\quad\left[R_{u_{i}}, R_{t}\right]=E_{v_{i}, t}-E_{t, u_{i}} \quad$ and $\left[R_{v_{i}}, R_{t}\right]=E_{u_{i}, t}-E_{t, v_{i}}$, we have

$$
\begin{aligned}
{\left[R_{u_{i}}+E_{t, u_{i}}-E_{v_{i}, t}, H\right] } & =-\omega_{0}\left(E_{t, u_{i}}-E_{v_{i}, t}\right)+\omega_{i} R_{u_{i}}-\omega_{0} R_{u_{i}}+\omega_{i}\left(E_{t, u_{i}}-E_{v_{i}, t}\right) \\
& =\left(-\omega_{0}+\omega_{i}\right)\left(R_{u_{i}}+E_{t, u_{i}}-E_{v_{i}, t}\right), \\
{\left[-R_{u_{i}}+E_{t, u_{i}}-E_{v_{i}, t}, H\right] } & =\left(\omega_{0}+\omega_{i}\right)\left(-R_{u_{i}}+E_{t, u_{i}}-E_{v_{i}, t}\right), \\
{\left[R_{v_{i}}+E_{u_{i}, t}-E_{t, v_{i}}, H\right] } & =\left(\omega_{0}-\omega_{i}\right)\left(R_{v_{i}}+E_{u_{i}, t}-E_{t, v_{i}}\right), \\
{\left[-R_{v_{i}}+E_{u_{i}, t}-E_{t, v_{i}}, H\right] } & =\left(-\omega_{0}-\omega_{i}\right)\left(-R_{v_{i}}+E_{u_{i}, t}-E_{t, v_{i}}\right) .
\end{aligned}
$$

Thus the roots of $\mathfrak{R}$ are $\pm \psi \pm \omega_{i}(i \geqq 0), \pm \omega_{i} \pm \omega_{j}(i \neq j)$; this means that $\mathfrak{R}$ is of type $D_{l+2}$. Since $\varepsilon^{*}$ maps $\psi \rightarrow-\psi, \omega_{0} \rightarrow-\omega_{0}$, and $\omega_{i} \rightarrow \omega_{i}(i \geqq 1)$, we see that a simple system of roots of $\mathfrak{L}^{\prime}$ (which is of type $D_{l+1}$, since its roots are $\pm \omega_{i} \pm \omega_{j}$ $(i \neq j))$ which is stabilized by $\varepsilon^{*}$ is $\omega_{l}-\omega_{l-1}, \ldots, \omega_{2}-\omega_{1}, \omega_{1}-\omega_{0}, \omega_{1}+\omega_{0}$. The projection of this simple system onto $\mathfrak{B}^{*}$ is $\omega_{l}-\omega_{l-1}, \ldots, \omega_{2}-\omega_{1}, \omega_{1}$, which is a simple system of roots of $\Sigma_{\varepsilon}$, i.e. (in this case), of roots of $\mathfrak{D}$. The corresponding simple system of roots of $\AA$ is $\psi-\omega_{l}, \omega_{l}-\omega_{l-1}, \ldots, \omega_{1}-\omega_{0}, \omega_{1}+\omega_{0}$. Thus $\Re$ has the Dynkin diagram

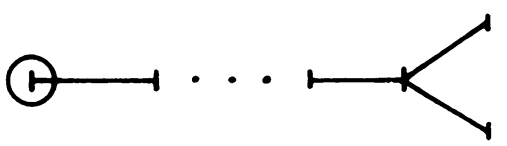

This diagram has two automorphisms, so $\left[\Gamma: \Gamma_{0}\right]=2$. Also $G$ has two components. The index of $G_{0}$ is $(l+2)-l=2$ since $\mathfrak{D}$ is of type $B_{l}$. The other graph automorphism of the diagram of $\mathfrak{R}$ corresponds to the identity automorphism of $\mathfrak{D}^{\prime}$, since the roots $\omega_{1}-\omega_{0}$ and $\omega_{1}+\omega_{0}$, which are interchanged by this automorphism, are both mapped to $\omega_{1}$ when the roots of $\mathfrak{L}^{\prime}$ are projected onto $\mathfrak{B S}^{*}$. Hence the other component $G_{1}$ of $G$ has index $(l+1)-l=1$.

(IV) $\mathfrak{J}=\mathfrak{S}\left(\Phi_{n}\right)$, the algebra of symmetric $n \times n$ matrices, where $n=2 l, l \geqq 2$. $\Im$ can be represented as the algebra of symmetric linear transformations of a vector space $\mathfrak{B}$ of dimension $2 l$ equipped with a nondegenerate symmetric bilinear form. We choose a basis $v_{1}, \ldots, v_{2 l}$ for $\mathfrak{B}$ with respect to which the form has matrix $\left(\begin{array}{cc}0 & I_{l} \\ I_{l} & 0\end{array}\right)$. By $E_{i j}$ we mean $E_{v_{i}, v_{j}}$ (in the above notation). Then $\Im$ consists of matrices of the form

$$
\left(\begin{array}{ll}
a_{11} & a_{12} \\
a_{21} & a_{22}
\end{array}\right)
$$


where the $a_{i j}$ 's are $l \times l$ matrices satisfying $a_{22}=a_{11}^{t}, a_{12}^{t}=a_{12}, a_{21}^{t}=a_{21}$. By [10, p. 254], $D$ consists of all mappings $X \mapsto[X, Y]$, where $Y$ is a matrix skew with respect to the form on $\mathfrak{B}$, i.e. $Y$ has matrix

$$
\left(\begin{array}{ll}
a_{11} & a_{12} \\
a_{21} & a_{22}
\end{array}\right),
$$

with $a_{11}=-a_{22}^{t}, a_{12}=-a_{12}^{t}, a_{21}=-a_{21}^{t}$. We denote the derivation $X \mapsto[X, Y]$ by ad $Y$. The mapping $Y \mapsto$ ad $Y$ is injective.

A basis for $\mathfrak{\Im}$ consists of the matrices $E_{i i}+E_{l+i, l+i}=K_{i}, E_{j i}+E_{i+l, j+l}(i \neq j)$, $E_{i+l, j}+E_{j+l, i}(i<j), E_{i+l, i}, E_{j, i+l}+E_{i, j+l}(i<j), E_{i, i+l}$. A basis for the set of skew matrices is $E_{i i}-E_{l+i, l+i}=H_{i}, E_{j i}-E_{i+l, j+l}(i \neq j), E_{i+l, j}-E_{j+l, i}(i<j), E_{j, i+l}-$ $E_{i, j+l}(i<j)$. A Cartan subalgebra for $\mathfrak{D}$ is $\mathfrak{W}=\left\{\sum_{i=1}^{l} \psi_{i}\right.$ ad $H_{\imath} \mid \psi_{\imath} \in \Phi$, all $\left.i\right\}$. With this choice of $\mathfrak{i}, \mathfrak{U}=\bigoplus \sum_{i=1}^{l} \Phi K_{i}$, so that $\mathfrak{S}=\left\{\sum_{i=1}^{l} \omega_{i} \cdot 2 R_{K_{i}}+\sum_{i=1}^{l} \psi_{i}\right.$ ad $H_{i} \mid \omega_{i}$, $\psi_{i} \in \Phi$, all $\left.i\right\}$. As before, we let $\omega_{1}, \psi_{1}, \ldots, \omega_{l}, \psi_{l}$ be the corresponding dual basis of $\mathfrak{S}^{*}$. We exhibit the roots of $\mathfrak{R}$ : if $H=\sum_{i=1}^{l} \omega_{i} \cdot 2 R_{K_{i}}+\sum_{i=1}^{l} \psi_{i}$ ad $H_{i}$, then

$$
\begin{array}{rlrl}
{\left[K_{i}, H\right]} & =2 \omega_{i} K_{i}, \\
{\left[E_{j i}+E_{i+l, j+l}, H\right]} & =\left(\omega_{i}+\omega_{j}+\psi_{i}-\psi_{j}\right)\left(E_{j i}+E_{i+l, j+l}\right) & (i \neq j), \\
{\left[E_{i+l, j}+E_{j+l, i}, H\right]} & =\cdot\left(\omega_{i}+\omega_{j}+\psi_{i}+\psi_{j}\right)\left(E_{i+l, j}+E_{j+l, i}\right) & (i \leqq j), \\
{\left[E_{j, i+l}+E_{i, j+l}, H\right]} & =\left(\omega_{i}+\omega_{j}-\psi_{i}-\psi_{j}\right)\left(E_{j, i+l}+E_{i, j+l}\right) & & (i \leqq j), \\
{\left[\bar{K}_{i}, H\right]} & =-2 \omega_{i} \bar{K}_{i}, \\
\left.\overline{\left[E_{j i}+E_{\imath+l, j+l}\right.}, H\right] & =\left(-\omega_{i}-\omega_{j}+\psi_{i}-\psi_{j}\right) \overline{\left(E_{j i}+E_{i+l, j+l}\right)} & & (i \neq j), \\
\left.\overline{\left[E_{i+l, j}+E_{j+l, i}\right.}, H\right] & =\left(-\omega_{i}-\omega_{j}+\psi_{i}+\psi_{j}\right)\left(\overline{E_{i+l, j}+E_{j+l, i}}\right) & (i \leqq j), \\
{\left[\overline{E_{j, i+l}+E_{i, j+l}}, H\right]} & =\left(-\omega_{i}-\omega_{j}-\psi_{i}-\psi_{j}\right)\left(\overline{\left.E_{j, i+l}+E_{i, j+l}\right)}\right. & (i \leqq j) .
\end{array}
$$

Every element of $\operatorname{Hom}_{\Phi}(\mathfrak{B}, \mathfrak{B})$ is uniquely expressible as the sum of a skew and a symmetric matrix. Thus $\operatorname{Hom}_{\Phi}(\mathfrak{B}, \mathfrak{B}) \cong \mathfrak{L}$, where the isomorphism is $X+Y \leftrightarrow$ $2 R_{X}+$ ad $Y$ for $X$ symmetric (i.e. in $\Im$ ) and $Y$ skew. This is easy to verify and allows us to find the roots of $\mathfrak{\Omega}$ whose root spaces lie in $\mathfrak{Q}$ more easily. For each $i, j$ let $e_{i j}$ denote the element of $\mathfrak{L}$ corresponding to $E_{i j} \in \operatorname{Hom}_{\Phi}(\mathfrak{B}, \mathfrak{B})$ under the above isomorphism. Then $2 R_{K_{i}}=e_{i i}+e_{l+i, l+i}$ and ad $H_{i}=e_{i i}-e_{l+i, l+i}$. Thus $H=\sum_{i=1}^{l}\left(\omega_{i}+\psi_{i}\right) e_{i i}+\left(\omega_{i}-\psi_{i}\right) e_{i+l, i+l}$. A basis for $\mathfrak{Q}$ consists of $2 R_{K_{i}}$, ad $H_{i}$, $e_{j i}(i \neq j), e_{i+l, j+l}(i \neq j), e_{i+l, j}($ all $i, j), e_{i, j+l}$ (all $\left.i, j\right)$. Then

$$
\begin{array}{rlrl}
{\left[e_{j i}, H\right]} & =\left(\omega_{i}+\psi_{i}-\omega_{j}-\psi_{j}\right) e_{j i} & & (i \neq j), \\
{\left[e_{i+l, j+l}, H\right]} & =\left(\omega_{j}-\psi_{j}-\omega_{i}+\psi_{i}\right) e_{i+l, j+l} & (i \neq j), \\
{\left[e_{i+l, j}, H\right]} & =\left(\omega_{j}+\psi_{j}-\omega_{i}+\psi_{i}\right) e_{i+l, j} & & (i \neq j), \\
{\left[e_{i+l, i}, H\right]} & =2 \psi_{i} e_{i+l, i}, & \\
{\left[e_{i, j+l}, H\right]} & =\left(\omega_{j}-\psi_{j}-\omega_{i}-\psi_{i}\right) e_{i, j+l} & & (i \neq j), \\
{\left[e_{i, i+l}, H\right]} & =-2 \psi_{i} e_{i, i+l} . &
\end{array}
$$


We thus see that the roots of $\Re$, listed in the same order as above, are

A. Roots $\rho$ such that $\rho\left(R_{1}\right)=1.2 \omega_{i}=\left(\omega_{i}+\psi_{i}\right)+\left(\omega_{i}-\psi_{i}\right), \quad\left(\omega_{i}+\psi_{i}\right)+\left(\omega_{j}-\psi_{j}\right)$ $(i \neq j),\left(\omega_{i}+\psi_{i}\right)+\left(\omega_{j}+\psi_{j}\right)(i \leqq j),\left(\omega_{i}-\psi_{i}\right)+\left(\omega_{j}-\psi_{j}\right)(i \leqq j)$.

B. Roots $\rho$ such that $\rho\left(R_{1}\right)=-1 .-2 \omega_{i}=-\left(\omega_{i}-\psi_{i}\right)-\left(\omega_{i}+\psi_{i}\right),-\left(\omega_{i}-\psi_{i}\right)-$ $\left(\omega_{j}+\psi_{j}\right)(i \neq j),-\left(\omega_{i}-\psi_{i}\right)-\left(\omega_{j}-\psi_{j}\right)(i \leqq j),-\left(\omega_{i}+\psi_{i}\right)-\left(\omega_{j}+\psi_{j}\right)(i \leqq j)$.

C. Roots $\rho$ such that $\rho\left(R_{1}\right)=0 .\left(\omega_{i}+\psi_{i}\right)-\left(\omega_{j}+\psi_{j}\right)(i \neq j),-\left(\omega_{i}-\psi_{i}\right)+\left(\omega_{j}-\psi_{j}\right)$ $(i \neq j),-\left(\omega_{i}-\psi_{i}\right)+\left(\omega_{j}+\psi_{j}\right)(i \neq j), 2 \psi_{i}=-\left(\omega_{i}-\psi_{i}\right)+\left(\omega_{i}+\psi_{i}\right),-\left(\omega_{i}+\psi_{i}\right)+\left(\omega_{j}-\psi_{j}\right)$ $(i \neq j),-2 \psi_{i}=-\left(\omega_{i}+\psi_{i}\right)+\left(\omega_{i}-\psi_{i}\right)$.

Thus the roots of $\mathscr{R}$ are all sums and differences of $\omega_{i}+\psi_{i}$ and $\omega_{i}-\psi_{i}$. This means that $\mathfrak{R}$ is of type $C_{2 l}$. The roots of $\mathfrak{L}^{\prime}$ are all differences of $\omega_{i}+\psi_{i}$ and $\omega_{i}-\psi_{i}$, so that $\mathfrak{L}^{\prime}$ is of type $A_{2 l-1}$. A simple system of roots of $\mathfrak{L}^{\prime}$ stabilizes by $\varepsilon^{*}$ (which sends $\omega_{i} \rightarrow-\omega_{i}$ and $\left.\psi_{i} \rightarrow \psi_{i}\right)$ is $\left(\omega_{1}+\psi_{1}\right)-\left(\omega_{2}+\psi_{2}\right),\left(\omega_{2}+\psi_{2}\right)-\left(\omega_{3}+\psi_{3}\right), \ldots,\left(\omega_{l-1}+\right.$ $\left.\psi_{l-1}\right)-\left(\omega_{l}+\psi_{l}\right), \quad 2 \psi_{l}=\left(\omega_{l}+\psi_{l}\right)-\left(\omega_{l}-\psi_{l}\right), \quad\left(\omega_{l}-\psi_{l}\right)-\left(\omega_{l-1}-\psi_{l-1}\right), \ldots,\left(\omega_{2}-\psi_{2}\right)-$ $\left(\omega_{1}-\psi_{1}\right)$. Here $\varepsilon^{*}$ interchanges the first and last of these roots, the second and second from last, etc. The corresponding simple system of roots of $\AA$ adds to the end of this simple system the root $2\left(\omega_{1}-\psi_{1}\right)$. Thus the Dynkin diagram of $\mathfrak{R}$ is

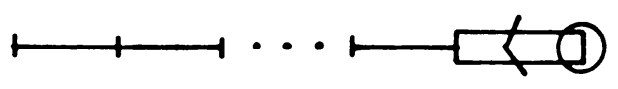

This has only one automorphism, so $\Gamma=\Gamma_{0}$.

The projections of the roots of $\mathfrak{R}^{\prime}$ onto $\mathfrak{B}^{*}$ are the roots $\pm \psi_{i} \pm \psi_{j}$ (all $i, j$ ). Thus $\Sigma_{\varepsilon}$ is of type $C_{l}$. The roots of $\Sigma_{\varepsilon}$ which are not roots of $\mathfrak{D}$ are $\pm 2 \psi_{i}$; for these are the projections of the only roots of $\mathfrak{L}^{\prime}$ whose root vectors have the form $R_{x}$, $x \in \mathfrak{\Im}$. Thus $\mathfrak{D}$ has roots $\pm \psi_{i} \pm \psi_{j}(i \neq j)$ and is of type $D_{l}$. The simple system of roots of $\Sigma_{\varepsilon}$ which we get from the above simple system of roots of $\mathfrak{L}^{\prime}$ is $\psi_{1}-\psi_{2}, \ldots$, $\psi_{l-1}-\psi_{l}, 2 \psi_{l}$. The corresponding simple system of roots of $\mathfrak{D}$ is $\psi_{1}-\psi_{2}, \ldots, \psi_{l-1}-$ $\psi_{l}, \psi_{l-1}+\psi_{l}$. As in example (II), we see that there are exactly two cosets of $W(\mathfrak{D})$ in $W\left(\Sigma_{\varepsilon}\right): W(\mathfrak{D})$ and $w_{2 \psi_{l}} W(\mathfrak{D})$. The second coset corresponds to the automorphism of the diagram of $\mathfrak{D}$ which interchanges $\psi_{l-1}-\psi_{l}$ and $\psi_{l-1}+\psi_{l}$ and fixes all the other simple roots. Thus $G$ has two components. The index of $G_{0}$ is $2 l-l=l$, while the index of the other component $G_{1}$ is $2 l-(l-1)=l+1$.

(V) $\mathfrak{\Im}=\mathfrak{S}\left(\Phi_{n}\right)$, the algebra of symmetric $n \times n$ matrices, and $n=2 l+1, l \geqq 1$. can be represented as the algebra of symmetric linear transformations of a vector space $\mathfrak{B}$ of dimension $2 l+1$ equipped with a nondegenerate symmetric bilinear form. We choose a basis $v_{0}, v_{1}, \ldots, v_{2 l}$ for $\mathfrak{B}$ with respect to which the form has matrix

$$
\left(\begin{array}{ccc}
1 & 0 & 0 \\
0 & 0 & I_{l} \\
0 & I_{l} & 0
\end{array}\right)
$$


As before, $E_{i j}$ means $E_{v_{i}, v_{j}}$. Then $\mathfrak{\Im}$ consists of linear transformations whose matrices have the form

$$
\left(\begin{array}{lll}
\lambda & u_{1} & u_{2} \\
u_{2}^{t} & a_{11} & a_{12} \\
u_{1}^{t} & a_{21} & a_{11}^{t}
\end{array}\right),
$$

where $\lambda \in \Phi, u_{1}, u_{2}$, are $1 \times l$ matrices, and $a_{i j}$ are $l \times l$ matrices satisfying $a_{12}=a_{12}^{t}$, $a_{21}=a_{21}^{t}$. Also the skew transformations of $\mathfrak{B}$ have matrices of the form

$$
\left(\begin{array}{ccc}
0 & u_{1} & u_{2} \\
-u_{2}^{t} & a_{11} & a_{12} \\
-u_{1}^{t} & a_{21} & -a_{11}^{t}
\end{array}\right),
$$

where $a_{12}=-a_{12}^{t}$ and $a_{21}=-a_{21}^{t}$. $\mathfrak{D}$ again consists of the mappings ad $Y$ for $Y$ skew. A basis for $\mathfrak{\Im}$ is $K_{0}=E_{00}, K_{i}=E_{i i}+E_{i+l, i+l}(i=1, \ldots, l), E_{j i}+E_{i+l, j+l}$ $(i \neq j ; i, j \geqq 1), E_{i+l, j}+E_{j+l, i}(1 \leqq i \leqq j), E_{j, i+l}+E_{i, j+l}(1 \leqq i \leqq j), E_{0, i}+E_{i+l, 0}(1 \leqq i)$, $E_{i, 0}+E_{0, i+l}(1 \leqq i)$. A Cartan subalgebra $\mathfrak{W}$ of $\mathfrak{D}$ is spanned by ad $H_{i}$, where $H_{i}=\left(E_{i i}-E_{i+l, i+l}\right), i \geqq 1$. With this choice of $\mathfrak{W}, \mathfrak{U}=\bigoplus \sum_{i=0} \Phi K_{i}$. Thus a typical element of $\mathfrak{S}_{\mathfrak{S}}$ is $H=\sum_{i=0}^{l} \omega_{i} \cdot 2 R_{K_{i}}+\sum_{i=1}^{l} \psi_{i}$ ad $H_{i}$. As before, we exhibit the roots of $\AA$ :

A. Roots $\rho$ such that $\rho\left(R_{1}\right)=1$.

$$
\begin{aligned}
{\left[K_{0}, H\right] } & =2 \omega_{0} K_{0}, & & \\
{\left[K_{i}, H\right] } & =2 \omega_{i} K_{i}=\left[\left(\omega_{i}+\psi_{i}\right)+\left(\omega_{i}-\psi_{i}\right)\right] K_{i} & & (1 \leqq i), \\
{\left[E_{j i}+E_{i+l, j+l}, H\right] } & =\left[\left(\omega_{i}+\psi_{i}\right)+\left(\omega_{j}-\psi_{j}\right)\right]\left(E_{j i}+E_{i+l, j+l}\right) & & (i \neq j ; i, j \geqq 1), \\
{\left[E_{i+l, j}+E_{j+l, i}, H\right] } & =\left[\left(\omega_{i}+\psi_{i}\right)+\left(\omega_{j}+\psi_{j}\right)\right]\left(E_{i+l, j}+E_{j+l, i}\right) & & (1 \leqq i \leqq j), \\
{\left[E_{j, i+l}+E_{i, j+l}, H\right] } & =\left[\left(\omega_{i}-\psi_{i}\right)+\left(\omega_{j}-\psi_{j}\right)\right]\left(E_{j, i+l}+E_{i, j+l}\right) & & (1 \leqq i \leqq j), \\
{\left[E_{0, i}+E_{i+l, 0}, H\right] } & =\left[\omega_{0}+\left(\omega_{i}+\psi_{i}\right)\right]\left(E_{0, i}+E_{i+l, 0}\right) & & (1 \leqq i), \\
{\left[E_{i, 0}+E_{0, i+l}, H\right] } & =\left[\omega_{0}+\left(\omega_{i}-\psi_{i}\right)\right]\left(E_{i, 0}+E_{0, i+l}\right) & & (1 \leqq i) .
\end{aligned}
$$

B. Roots $\rho$ such that $\rho\left(R_{1}\right)=-1$. (We obtain these by applying $\varepsilon^{*}$ to the roots listed above. $)-2 \omega_{0},-\left(\omega_{i}-\psi_{i}\right)-\left(\omega_{i}+\psi_{i}\right)(1 \leqq i),-\left(\omega_{i}-\psi_{i}\right)-\left(\omega_{1}+\psi_{j}\right)(i \neq j ; i, j \geqq 1)$, $-\left(\omega_{i}-\psi_{i}\right)-\left(\omega_{j}-\psi_{j}\right) \quad(1 \leqq i \leqq j), \quad-\left(\omega_{i}+\psi_{i}\right)-\left(\omega_{j}+\psi_{j}\right) \quad(1 \leqq i \leqq j), \quad-\omega_{0}-\left(\omega_{i}-\psi_{i}\right)$ $(1 \leqq i),-\omega_{0}-\left(\omega_{i}+\psi_{i}\right)(1 \leqq i)$.

C. As before we have a natural isomorphism between $\operatorname{Hom}_{\Phi}(\mathfrak{B}, \mathfrak{B})$ and $\mathfrak{L}$ given by $X+Y \leftrightarrow 2 R_{X}+$ ad $Y$ for $X$ in $\mathfrak{J}$ and $Y$ skew. If $e_{i j}$ is the element of $\mathfrak{Q}$ corresponding to $E_{i j} \in \operatorname{Hom}_{\Phi}(\mathfrak{B}, \mathfrak{B})$, then

$$
H=\omega_{0} e_{00}+\sum_{i=1}^{l}\left(\omega_{i}+\psi_{i}\right) e_{i i}+\left(\omega_{i}-\psi_{i}\right) e_{i+l, i+l}
$$


and we can list the roots $\rho$ of $\Re$ such that $\rho\left(R_{1}\right)=0$.

$$
\begin{aligned}
{\left[e_{j i}, H\right] } & =\left[\left(\omega_{i}+\psi_{i}\right)-\left(\omega_{j}+\psi_{j}\right)\right] e_{j i} & & (1 \leqq i<j), \\
{\left[e_{i+l, j+l}, H\right] } & =\left[-\left(\omega_{i}-\psi_{i}\right)+\left(\omega_{j}-\psi_{j}\right)\right] e_{i+l, j+l} & & (1 \leqq i<j), \\
{\left[e_{i+l, j}, H\right] } & =\left[-\left(\omega_{i}-\psi_{i}\right)+\left(\omega_{j}+\psi_{j}\right)\right] e_{i+l, j} & & (i \neq j ; i, j \geqq 1), \\
{\left[e_{i+l, i}, H\right] } & =\left[-\left(\omega_{i}-\psi_{i}\right)+\left(\omega_{i}+\psi_{i}\right)\right] e_{i+l, i}=2 \psi_{i} e_{i+l, i} & & (1 \leqq i), \\
{\left[e_{i, j+l}, H\right] } & =\left[-\left(\omega_{i}+\psi_{i}\right)+\left(\omega_{j}-\psi_{j}\right)\right] e_{i, j+l} & & (i \neq j ; i, j \geqq 1), \\
{\left[e_{i, i+l}, H\right] } & =\left[-\left(\omega_{i}+\psi_{i}\right)+\left(\omega_{i}-\psi_{i}\right)\right] e_{i, i+l}=-2 \psi_{i} e_{i, i+l} & & (1 \leqq i), \\
{\left[e_{0, i}, H\right] } & =\left[-\omega_{0}+\left(\omega_{i}+\psi_{i}\right)\right] e_{0, i} & & (1 \leqq i), \\
{\left[e_{i, 0}, H\right] } & =\left[\omega_{0}-\left(\omega_{i}+\psi_{i}\right)\right] e_{i, 0} & & (1 \leqq i), \\
{\left[e_{i+l, 0}, H\right] } & =\left[\omega_{0}-\left(\omega_{i}-\psi_{i}\right)\right] e_{i+l, 0} & & (1 \leqq i), \\
{\left[e_{0, i+l}, H\right] } & =\left[-\omega_{0}+\left(\omega_{i}-\psi_{i}\right)\right] e_{0, i+l} & & (1 \leqq i) .
\end{aligned}
$$

The roots of $\mathfrak{R}$ are all sums and differences of $\omega_{0}, \omega_{i}+\psi_{i}, \omega_{i}-\psi_{i}$. Thus $\mathfrak{R}$ is of type $C_{2 l+1}$. The roots of $\mathfrak{L}^{\prime}$ are all differences of these terms, so that $\mathfrak{L}^{\prime}$ is of type $A_{2 l}$. A simple system of roots of $\mathfrak{L}^{\prime}$ stabilized by $\varepsilon^{*}$ is $\left(\omega_{1}+\psi_{1}\right)-\left(\omega_{2}+\psi_{2}\right), \ldots$, $\left(\omega_{l-1}+\psi_{l-1}\right)-\left(\omega_{l}+\psi_{l}\right), \quad\left(\omega_{l}+\psi_{l}\right)-\omega_{0}, \omega_{0}-\left(\omega_{l}-\psi_{l}\right), \quad\left(\omega_{l}-\psi_{l}\right)-\left(\omega_{l-1}-\psi_{l-1}\right), \ldots$ $\left(\omega_{2}-\psi_{2}\right)-\left(\omega_{1}-\psi_{1}\right)$. Here $\varepsilon^{*}$ interchanges the first and last of these roots, the second and second from last, etc. The corresponding simple system of roots of $\Re$ is obtained by adding $2\left(\omega_{1}-\psi_{1}\right)$ to the end of this list. Thus the diagram of $\mathfrak{R}$ is

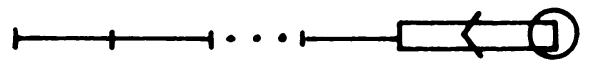

Again $\Gamma=\Gamma_{0}$.

The roots of $\Sigma_{\varepsilon}$ are $\pm \psi_{i} \pm \psi_{j}(i \neq j), \pm \psi_{i}, \pm 2 \psi_{i}$. So $\Sigma_{\varepsilon}$ is of type $B C_{l}$. The only roots of $\Sigma_{\varepsilon}$ which are not roots of $\mathfrak{D}$ are $\pm 2 \psi_{i}$, since these are the projections of roots of $\mathfrak{Q}^{\prime}$ whose root vectors are of the form $R_{x}$. Thus $\mathfrak{D}$ is of type $B_{l}$. The simple system of roots of $\Sigma_{\varepsilon}$ corresponding to the above simple system of roots of $\mathfrak{R}^{\prime}$ is $\psi_{1}-\psi_{2}, \ldots, \psi_{l-1}-\psi_{l}, \psi_{l}$. These roots are also roots of $\mathfrak{D}$ and since symmetries in the simple roots generate the Weyl group, $W(\mathfrak{D})=W\left(\Sigma_{\varepsilon}\right)$. Thus $\Gamma_{0} \cap G=G_{0}$, and $G$ has only one component. The index of $G_{0}$ is $(2 l+1)-l=l+1$.

(VI) $\mathfrak{S}=\Phi_{n}^{+}$, the Jordan algebra of all $n \times n$ matrices over $\Phi$. As before, $\mathfrak{D}$ consists of all mappings $X \rightarrow[X, Y], Y \in \Phi_{n}$. We call this derivation ad $Y$. The mapping of $\Phi_{n}^{-}$(the Lie algebra of all $n \times n$ matrices) onto $\mathfrak{D}$ defined by $Y \mapsto$ ad $Y$ is a Lie homomorphism whose kernel is the center of $\Phi_{n}^{-}$, the set of scalar matrices. If $\mathfrak{B}$ is the set of matrices of trace zero, then $Y \mapsto$ ad $Y$ is an isomorphism of $\mathfrak{B}$ onto $\mathfrak{D}$. Thus a Cartan subalgebra $\mathfrak{B}$ of $\mathfrak{D}$ is spanned by ad $h_{i}$, where $h_{i}=$ ad $\left(e_{i i}-e_{n n}\right), i=1, \ldots, n-1$; here by $e_{i j}$ we mean the matrix with 1 in the $i j$-position and 0 elsewhere. It is easy to see that with this choice of $\mathfrak{W}, \mathfrak{A}=\Phi e_{11} \oplus \cdots \oplus \Phi e_{n n}$;

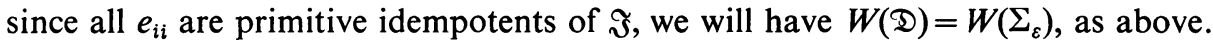


Let $H=\sum_{i=1}^{n} \omega_{i} \cdot 2 R_{e_{i}}+\sum_{i=1}^{n-1} \psi_{i}$ ad $h_{i}$ be a typical element of $\mathfrak{E}$, and let $\gamma=\sum_{i=1}^{n-1} \psi_{i}$. We exhibit the roots of $\mathfrak{R}$ :

A. Roots $\rho$ such that $\rho\left(R_{1}\right)=1$.

$$
\begin{array}{rlrl}
{\left[e_{i i}, H\right]} & =2 \omega_{i} e_{i i}=\left[\left(\omega_{i}+\psi_{i}\right)-\left(-\omega_{i}+\psi_{i}\right)\right] e_{i i} & & (i<n), \\
{\left[e_{n n}, H\right]} & =2 \omega_{n} e_{n n}=\left[\left(\omega_{n}-\gamma\right)-\left(-\omega_{n}-\gamma\right)\right] e_{n n}, & \\
{\left[e_{i n}, H\right]} & =\left[\left(\omega_{n}-\gamma\right)-\left(-\omega_{i}+\psi_{i}\right)\right] e_{i n} & & (i<n), \\
{\left[e_{n j}, H\right]} & =\left[\left(\omega_{j}+\psi_{j}\right)-\left(-\omega_{n}-\gamma\right)\right] e_{n j} & & (j<n), \\
{\left[e_{i j}, H\right]} & =\left[\left(\omega_{j}+\psi_{j}\right)-\left(-\omega_{i}-\psi_{i}\right)\right] e_{i j} & & (i, j, n \text { distinct }) .
\end{array}
$$

B. Roots $\rho$ such that $\rho\left(R_{1}\right)=-1 .-2 \omega_{i}=\left(-\omega_{i}+\psi_{i}\right)-\left(\omega_{i}+\psi_{i}\right)(i<n),-2 \omega_{n}=$ $\left(-\omega_{n}-\gamma\right)-\left(\omega_{n}-\gamma\right), \quad\left(-\omega_{n}-\gamma\right)-\left(\omega_{i}+\psi_{i}\right) \quad(i<n), \quad\left(-\omega_{j}+\psi_{j}\right)-\left(\omega_{n}-\gamma\right) \quad(j<n)$, $\left(-\omega_{j}+\psi_{j}\right)-\left(\omega_{i}+\psi_{i}\right)(i, j, n$ distinct $)$.

C. Roots $\rho$ such that $\rho\left(R_{1}\right)=0$. It is not hard to check that

$$
\begin{array}{rlrl}
{\left[2 R_{e_{i j}}, 2 R_{e_{k k}}\right]} & =\operatorname{ad}\left[e_{i j}, e_{k k}\right]=\left(\delta_{j k}-\delta_{i k}\right) \operatorname{ad} e_{i j}, \\
{\left[\operatorname{ad} e_{i j}, 2 R_{e_{k k}}\right]} & =2 R_{\left[e_{i j}, e_{k k}\right]}=\left(\delta_{j k}-\delta_{i k}\right) 2 R_{e_{i j}}, \\
{\left[2 R_{e_{i n}}, \operatorname{ad}\left(e_{k k}-e_{n n}\right)\right]} & =\left(-1-\delta_{i k}\right) 2 R_{e_{i n}} & (i, k<n), \\
{\left[2 R_{e_{n j}}, \operatorname{ad}\left(e_{k k}-e_{n n}\right)\right]} & =\left(1+\delta_{j k}\right) 2 R_{e_{n j}} & (j, k<n), \\
{\left[2 R_{e_{i j}}, \operatorname{ad}\left(e_{k k}-e_{n n}\right)\right]} & =\left(\delta_{k j}-\delta_{k i}\right) 2 R_{e_{i j}} & (i \neq j ; i, j, k<n), \\
{\left[\operatorname{ad} e_{i n}, \operatorname{ad}\left(e_{k k}-e_{n n}\right)\right]} & =\left(-1-\delta_{i k}\right) \operatorname{ad} e_{i n} & (i, k<n), \\
{\left[\operatorname{ad} e_{n j}, \operatorname{ad}\left(e_{k k}-e_{n n}\right)\right]} & =\left(1+\delta_{j k}\right) \operatorname{ad} e_{n j} & & (j, k<n), \\
{\left[\operatorname{ad} e_{i j}, \operatorname{ad}\left(e_{k k}-e_{n n}\right)\right]} & =\left(\delta_{k j}-\delta_{k i}\right) \operatorname{ad} e_{i j} & & (i \neq j ; i, j, k<n) .
\end{array}
$$

Applying these, we see that

$$
\begin{aligned}
{\left[2 R_{e_{i n}}+\operatorname{ad} e_{i n}, H\right] } & =\left[\left(\omega_{n}-\gamma\right)-\left(\omega_{i}+\psi_{i}\right)\right]\left(2 R_{e_{i n}}+\operatorname{ad} e_{i n}\right) & & (i<n), \\
{\left[-2 R_{e_{i n}}+\operatorname{ad} e_{i n}, H\right] } & =\left[\left(-\omega_{n}-\gamma\right)-\left(-\omega_{i}+\psi_{\imath}\right)\right]\left(-2 R_{e_{i n}}+\operatorname{ad} e_{i n}\right) & & (i<n), \\
{\left[2 R_{e_{n j}}+\operatorname{ad} e_{n j}, H\right] } & =\left[\left(\omega_{j}+\psi_{j}\right)-\left(\omega_{n}-\gamma\right)\right]\left(2 R_{e_{n j}}+\operatorname{ad} e_{n j}\right) & & (j<n), \\
{\left[-2 R_{e_{n j}}+\operatorname{ad} e_{n j}, H\right] } & =\left[\left(-\omega_{j}+\psi_{j}\right)-\left(-\omega_{n}-\gamma\right)\right]\left(-2 R_{e_{n j}}+\operatorname{ad} e_{n j}\right) & & (j<n), \\
{\left[2 R_{e_{i j}}+\operatorname{ad} e_{i j}, H\right] } & =\left[\left(\omega_{j}+\psi_{j}\right)-\left(\omega_{i}+\psi_{i}\right)\right]\left(2 R_{e_{i j}}+\operatorname{ad} e_{i j}\right) & & (i, j, n \text { distinct }), \\
{\left[-2 R_{e_{i j}}+\operatorname{ad} e_{i j}, H\right] } & =\left[\left(-\omega_{j}+\psi_{j}\right)-\left(-\omega_{i}+\psi_{i}\right)\right]\left(-2 R_{e_{i j}}+\operatorname{ad} e_{i j}\right) & & (i, j, n \text { distinct }) .
\end{aligned}
$$

Thus the roots of $\mathfrak{R}$ are all differences of $\omega_{i}+\psi_{i}(i<n),-\omega_{i}+\psi_{i}(i<n), \omega_{n}-\gamma$ and $-\omega_{n}-\gamma$. Thus $\mathfrak{R}$ is of type $A_{2 n-1}$. The roots of $\mathfrak{R}^{\prime}$ are all differences of $\omega_{i}+\psi_{i}$ $(i<n)$ and $\omega_{n}-\gamma$, together with all differences of $-\omega_{i}+\psi_{i}(i<n)$ and $-\omega_{n}-\gamma$. Thus $\mathfrak{L}^{\prime}$ is of type $A_{n-1} \oplus A_{n-1}$. A simple system of roots of $\mathfrak{R}^{\prime}$ stabilized by $\varepsilon^{*}$, augmented in the usual manner to give a simple system of roots of $\AA$, is $\left(\omega_{1}+\psi_{1}\right)-\left(\omega_{2}+\psi_{2}\right), \ldots,\left(\omega_{n-2}+\psi_{n-2}\right)-\left(\omega_{n-1}+\psi_{n-1}\right), \quad\left(\omega_{n-1}+\psi_{n-1}\right)-\left(\omega_{n}-\gamma\right)$, $\left(\omega_{n}-\gamma\right)-\left(-\omega_{1}+\psi_{1}\right), \quad\left(-\omega_{1}+\psi_{1}\right)-\left(-\omega_{2}+\psi_{2}\right), \ldots,\left(-\omega_{n-2}+\psi_{n-2}\right)-\left(-\omega_{n-1}+\right.$ $\left.\psi_{n-1}\right),\left(-\omega_{n-1}+\psi_{n-1}\right)-\left(-\omega_{n}-\gamma\right)$. Here the root $\rho_{1}$ which has been added to 
the simple system of roots of $\mathfrak{L}^{\prime}$ is the middle root, $\left(\omega_{n}-\gamma\right)-\left(-\omega_{1}+\psi_{1}\right)$. Thus the Dynkin diagram of $\Re$ is

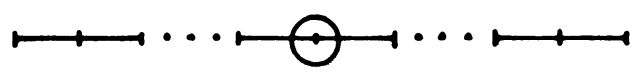

which has two automorphisms. This means that $\left[\Gamma: \Gamma_{0}\right]=\left[G: G_{0}\right]=2$. In the above list of simple roots, $\varepsilon^{*}$ interchanges the first and $(n+1)$ th, the second and $(n+2)$ th, etc. The roots of $\Sigma_{\varepsilon}$ (hence of $\left.\mathfrak{D}\right)$ are all differences of $\psi_{1}, \ldots, \psi_{n-1},-\gamma$; hence $\Sigma_{\varepsilon}$ is of type $A_{n-1}$. The simple system of roots of $\Sigma_{\varepsilon}$ obtained by projecting the above simple system of roots of $\mathfrak{R}^{\prime}$ is $\psi_{1}-\psi_{2}, \psi_{2}-\psi_{3}, \ldots, \psi_{n-2}-\psi_{n-1}, \psi_{n-1}-(-\gamma)$. The effect on this system of the nontrivial automorphism of the Dynkin diagram of $\AA$ (which interchanges $\left(\omega_{1}+\psi_{1}\right)-\left(\omega_{2}+\psi_{2}\right)$ and $\left(-\omega_{n-1}+\psi_{n-1}\right)-\left(-\omega_{n}-\gamma\right)$, etc.) is to interchange $\psi_{1}-\psi_{2}$ and $\psi_{n-1}-(-\gamma), \psi_{2}-\psi_{3}$ and $\psi_{n-2}-\psi_{n-1}$, etc. The number of orbits of this automorphism of the Dynkin diagram of $\mathfrak{D}$ is $[n / 2]$. Thus the index of $G_{0}$ is $(2 n-1)-(n-1)=n$, while the index of the other component $G_{1}$ of $G$ is $[2 n / 2]-[n / 2]=n-[n / 2]=[(n+1) / 2]$.

(VII) $\mathfrak{I}=\mathfrak{S}\left(\Phi_{2 n}, J\right), n \geqq 3$, i.e. $\mathfrak{\Im}$ is the set of $2 n \times 2 n$ matrices symmetric under the involution $J: X \rightarrow S^{-1} X S$, where $S=\operatorname{diag}\{Q, Q, \ldots, Q\}$ (there are $n Q$ 's here) and $Q$ is the $2 \times 2$ matrix $\left(\begin{array}{cc}0 & 1 \\ -1 & 0\end{array}\right)$. For purposes of notation, we divide matrices up into $n^{2} 2 \times 2$ blocks. By $I_{i j}$ we mean the $2 n \times 2 n$ matrix which has $\left(\begin{array}{ll}1 & 0 \\ 0 & 1\end{array}\right)$ in the $i j$-block and $\left(\begin{array}{ll}0 & 0 \\ 0 & 0\end{array}\right)$ elsewhere; thus we regard $\Phi_{2 n}$ as the algebra of all $n \times n$ matrices over $\Phi_{2}$. We note that $Q^{-1}=Q^{t}$. If $X=\sum_{i, j} X_{i j} I_{i j} \quad\left(X_{i j} \in \Phi_{2}\right)$, then $X^{J}=$ $\sum_{i, j} Q^{-1} X_{j i}^{t} Q I_{i j}$. Also $Q^{-1}\left(\begin{array}{cc}a & b \\ c & d\end{array}\right) Q=\left(\begin{array}{cc}d & -b \\ -c & -b\end{array}\right)$. From these facts we can see that a basis for $\Im$ consists of $I_{i i}, P_{i j}=\left(\begin{array}{ll}0 & 0 \\ 0 & 1\end{array}\right) I_{i j}+\left(\begin{array}{ll}1 & 0 \\ 0 & 0\end{array}\right) I_{j i} \quad(i \neq j), \quad R_{i j}=\left(\begin{array}{ll}0 & 1 \\ 0 & 0\end{array}\right) I_{i j}+\left(\begin{array}{cc}0 & -1 \\ 0 & 0\end{array}\right) I_{j i}$ $(i<j), S_{i j}=\left(\begin{array}{ll}0 & 0 \\ 1 & 0\end{array}\right) I_{i j}+\left(\begin{array}{rr}0 & 0 \\ -1 & 0\end{array}\right) I_{j i}(i<j)$. As before, $\mathfrak{D}$ consists entirely of mappings ad $A$, where $A \in \Phi_{2 n}$ and $A^{J}=-A$. The mapping $A \mapsto$ ad $A$ from the set of $J$-skew matrices onto $\mathfrak{D}$ is a Lie isomorphism. A Cartan subalgebra for the algebra of $J$-skew matrices is spanned by $H_{i}=\left(\begin{array}{rr}-1 & 0 \\ 0 & 1\end{array}\right) I_{i i}(i=1, \ldots, n)$. Thus we can take $\mathfrak{B}=\left\{\sum_{i=1}^{n} \psi_{i}\right.$ ad $\left.H_{i} \mid \psi_{i} \in \Phi\right\}$. It is not hard to see that $\mathfrak{A}$ is spanned by all $I_{i i}$, which are primitive idempotents of $\mathfrak{\Im}$. Hence in this case we will have $W(\mathfrak{D})=$ $W\left(\Sigma_{\varepsilon}\right)$. We exhibit the roots of $\mathfrak{R}$ : if $H=\sum_{i=1}^{n} \omega_{i} \cdot 2 R_{I_{i i}}+\sum_{i=1}^{n} \psi_{i}$ ad $H_{i}$, then we have

A. Roots $\rho$ such that $\rho\left(R_{1}\right)=1$.

$$
\begin{array}{ll}
{\left[P_{i j}, H\right]=\left[\left(\omega_{i}-\psi_{i}\right)+\left(\omega_{j}+\psi_{j}\right)\right] P_{i j}} & (i \neq j), \\
{\left[R_{i j}, H\right]=\left[\left(\omega_{i}+\psi_{i}\right)+\left(\omega_{j}+\psi_{j}\right)\right] R_{i j}} & (i<j), \\
{\left[S_{i j}, H\right]=\left[\left(\omega_{i}-\psi_{i}\right)+\left(\omega_{j}-\psi_{j}\right)\right] S_{i j}} & (i<j) .
\end{array}
$$

B. Roots $\rho$ such that $\rho\left(R_{1}\right)=-1 .-\left(\omega_{i}+\psi_{i}\right)-\left(\omega_{j}-\psi_{j}\right) \quad(i \neq j),-\left(\omega_{i}-\psi_{i}\right)-$ $\left(\omega_{j}-\psi_{j}\right)(i<j),-\left(\omega_{i}+\psi_{i}\right)-\left(\omega_{j}+\psi_{j}\right)(i<j)$.

C. As before, we have an isomorphism between $\Phi_{2 n}^{-}$and $\mathfrak{L}$ given as follows: 
if $X^{J}=X$ and $Y^{J}=-Y$, then $X+Y \leftrightarrow 2 R_{X}+$ ad $Y$. Under this isomorphism $H \in \mathfrak{Q}$ corresponds to

$$
\sum_{i=1}^{n}\left(\begin{array}{cc}
\omega_{i}-\psi_{i} & 0 \\
0 & \omega_{i}+\psi_{i}
\end{array}\right) I_{i i} \cdot
$$

If $E_{i}$ is the element of $\mathfrak{Q}$ corresponding to $\left(\begin{array}{ll}0 & 1 \\ 0 & 0\end{array}\right) I_{i i}, F_{i}$ to $\left(\begin{array}{cc}0 & 0 \\ -1 & 0\end{array}\right) I_{i i}, I_{i j}^{11}$ to $\left(\begin{array}{ll}1 & 0 \\ 0 & 0\end{array}\right) I_{i j}$, $I_{i j}^{12}$ to $\left(\begin{array}{ll}0 & 1 \\ 0 & 0\end{array}\right) I_{i j}, I_{i j}^{21}$ to $\left(\begin{array}{cc}0 & 0 \\ 1 & 0\end{array}\right) I_{i j}$, and $I_{i j}^{22}$ to $\left(\begin{array}{ll}0 & 0 \\ 0 & 1\end{array}\right) I_{i j}$, then we have for the roots $\rho$ such that $\rho\left(R_{1}\right)=0$,

$$
\begin{aligned}
& {\left[E_{i}, H\right]=2 \psi_{i} E_{i}=\left[\left(\omega_{i}+\psi_{i}\right)-\left(\omega_{i}-\psi_{i}\right)\right] E_{i},} \\
& {\left[F_{i}, H\right]=-2 \psi_{i} F_{i}=\left[\left(\omega_{i}-\psi_{i}\right)-\left(\omega_{i}+\psi_{i}\right)\right] F_{i},} \\
& {\left[I_{i j}^{11}, H\right]=\left[\left(\omega_{j}-\psi_{j}\right)-\left(\omega_{i}-\psi_{i}\right)\right] I_{i j}^{11} \quad(i \neq j),} \\
& {\left[I_{i j}^{12}, H\right]=\left[\left(\omega_{j}+\psi_{j}\right)-\left(\omega_{i}-\psi_{i}\right)\right] I_{i j}^{12} \quad(i \neq j),} \\
& {\left[I_{i j}^{21}, H\right]=\left[\left(\omega_{j}-\psi_{j}\right)-\left(\omega_{i}+\psi_{i}\right)\right] I_{i j}^{21} \quad(i \neq j),} \\
& {\left[I_{i j}^{22}, H\right]=\left[\left(\omega_{j}+\psi_{j}\right)-\left(\omega_{i}+\psi_{i}\right)\right] I_{i j}^{22} \quad(i \neq j) .}
\end{aligned}
$$

Thus the roots of $\mathcal{R}$ are all sums and differences of distinct terms $\omega_{i}+\psi_{i}$ and $\omega_{i}-\psi_{i}$. Hence $\mathfrak{I}$ is of type $D_{2 n}$. The roots of $\mathfrak{L}^{\prime}$ are all differences of these terms, so $\mathfrak{R}^{\prime}$ is of type $A_{2 n-1}$. A simple system of $\mathfrak{L}^{\prime}$ stabilized by $\varepsilon^{*}$ is $\left(\omega_{1}+\psi_{1}\right)-\left(\omega_{2}+\psi_{2}\right), \ldots,\left(\omega_{n-1}+\psi_{n-1}\right)-\left(\omega_{n}+\psi_{n}\right), \quad\left(\omega_{n}+\psi_{n}\right)-\left(\omega_{n}-\psi_{n}\right), \quad\left(\omega_{n}-\psi_{n}\right)$ $-\left(\omega_{n-1}-\psi_{n-1}\right), \ldots,\left(\omega_{2}-\psi_{2}\right)-\left(\omega_{1}-\psi_{1}\right)$. Here $\varepsilon^{*}$ interchanges the first and last roots, the second and second from last, etc. One adds to the list the root $\left(\omega_{2}-\psi_{2}\right)$ $+\left(\omega_{1}-\psi_{1}\right)$ to get the corresponding simple system of roots of $\AA$. Thus $\AA$ has Dynkin diagram

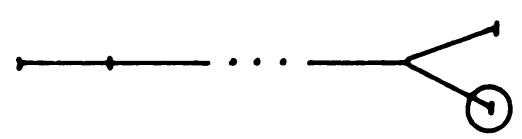

Since $n \geqq 3,2 n \geqq 6$ and this has only one automorphism. As a result, $\Gamma=\Gamma_{0}$ and hence $G=G_{0}$. The roots of $\Sigma_{\varepsilon}$ (i.e. of $\left.\mathfrak{D}\right)$ are $\pm \psi_{i} \pm \psi_{j}(i \neq j), \pm 2 \psi_{j}$, so that $\mathfrak{D}$ is of type $C_{n}$. The simple system of roots of $\mathfrak{D}$ derived from the above simple system of roots of $\mathfrak{L}^{\prime}$ is $\psi_{1}-\psi_{2}, \psi_{2}-\psi_{3}, \ldots, \psi_{n-1}-\psi_{n}, 2 \psi_{n}$. The index of $G_{0}$ is $2 n-n=n$.

(VIII) $\mathfrak{\Im}=\mathfrak{H}\left(\mathfrak{D}_{3}\right)$, the exceptional simple Jordan algebra of all $3 \times 3$ hermitian matrices over the octonian algebra $\mathfrak{D}$. We saw following Proposition 10 that $\mathfrak{A}$ is of type $E_{7}$. Since the Dynkin diagram of $\AA$ has no nontrivial automorphisms, $\Gamma=\Gamma_{0} . \mathfrak{D}$ is of type $F_{4}[7$, p. 35]; since the Dynkin diagram of $\mathfrak{D}$ has no nontrivial automorphisms, $G=G_{0}$. Also the index of $G_{0}$ is $7-4=3$ (this has been shown for all characteristics $\neq 2,3$ by Smith [14]). It is known [7, p. 36] that $\mathfrak{A}$ is spanned by primitive idempotents. Thus $\Sigma_{\varepsilon}$ is also of type $F_{4}$.

A table summarizing examples (II) through (VIII) appears in [13, p. 503].

Appendix. In the proof of Theorem 3, we used the following fact: if $e$ and $f$ are two primitive idempotents of $\mathfrak{\Im}=\Phi 1 \oplus \mathfrak{B}$, the (simple) Jordan algebra of a 
space equipped with a nondegenerate symmetric bilinear form, then $U_{2 e-1} U_{2 f-1} \in$ Aut $_{0} \Im$. In the proof of Theorem 5 we used similar facts about the three-dimensional simple Jordan algebra $\mathfrak{B}$ over $\Phi$ : that the set of primitive idempotents of $\mathfrak{B}$ is an irreducible algebraic set and that if $\mathfrak{B}$ is a subalgebra of a Jordan algebra

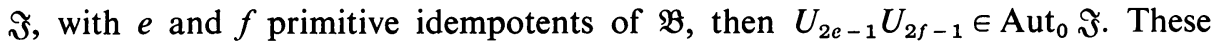
facts are corollaries of the following:

THEOREM. Let $\Im$ be a simple Jordan algebra over $\Phi$, an algebraically closed field of characteristic $p$, where $p=0$ or $p$ is bigger than or equal to the degree of $\Im$. Then the set of primitive idempotents of $\Im$ is an irreducible algebraic set.

This theorem was first proven by Helwig in [5]; his method was simply to examine each of the simple Jordan algebras as given by the classification theory, using special considerations for each. Our proof, except possibly at one point, will not use the classification theory.

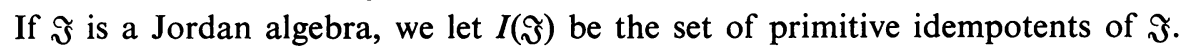
In general, if $e^{.2}=e$, then $U_{2 e-1} \in$ Aut $\Im$. For $U_{2 e-1}$ is in the structure group of $\Im$ and $(2 e-1)^{2}=4 e-4 e+1=1$, so that $1 U_{2 e-1}=1$. If $x \in \Im$, then

$$
\begin{aligned}
x U_{2 e-1} & =2(x .(2 e-1)) \cdot(2 e-1)-x \cdot(2 e-1)^{\cdot 2} \\
& =8(x . e) \cdot e-4 x \cdot e-4 x \cdot e+2 x-x \\
& =8(x . e) . e-8 x . e+x .
\end{aligned}
$$

The next three propositions are sufficient for the applications in the proofs of Theorems 3 and 5 cited above.

Proposition 1. Let $\mathfrak{\Im}$ be a Jordan algebra and $\mathfrak{B}$ a subalgebra. Suppose that $I(\mathfrak{B})$ is an irreducible algebraic set and $e, f \in I(\mathfrak{B})$. Then $U_{2 e-1} U_{2 f-1} \in \mathrm{Aut}_{0} \mathfrak{\Im}$.

Proof. We have seen that for $e \in I(\mathfrak{B}), U_{2 e-1} \in$ Aut $\mathfrak{~}$. Fix $e \in I(\mathfrak{B})$ and consider the polynomial map of $I(\mathfrak{B}) \rightarrow$ Aut $\mathfrak{J}$ defined by $f \mapsto U_{2 e-1} U_{2 f-1}$. The image of $I(\mathfrak{B})$ is an irreducible subset of Aut $\Im$ which contains 1 (since $U_{2 e-1} U_{2 e-1}=1$ ). Hence it is contained in $\mathrm{Aut}_{0} \mathfrak{\Im}$.

Proposition 2. Let $\mathfrak{B}$ be a vector space of dimension at least 2 with a nondegenerate symmetric bilinear form ( , ). Let $I(\mathfrak{B})=\{v \in \mathfrak{B} \mid(v, v)=1\}$. Then $I(\mathfrak{B})$ is an irreducible algebraic set.

Proof. Choose a basis $v_{1}, \ldots, v_{n}$ for $\mathfrak{B}$ and let $\left(\alpha_{i j}\right)$ be the matrix of the form with respect to this basis. If $v=\sum_{i=1}^{n} \lambda_{i} v_{i}$, then $v \in I(\mathfrak{B})$ if and only if $\sum_{i j} \alpha_{i j} \lambda_{i} \lambda_{j}-1$ $=0$. Thus $I(\mathfrak{B})$ is an algebraic set. To show it is irreducible, we only have to show that the polynomial $F\left(\lambda_{1}, \ldots, \lambda_{n}\right)=\sum_{i, j=1}^{n} \alpha_{i j} \lambda_{i} \lambda_{j}-1$ is irreducible. Suppose otherwise. Degree considerations show that proper factors of $F(\lambda)$ have degree one. Thus we have assumed that $F(\lambda)=(l(\lambda)+\alpha)(m(\lambda)+\beta)$ where $l, m$ are nonzero linear polynomials and $\alpha, \beta$ are scalars. Equating terms of degree zero, we find that $\alpha \beta=-1$. If we replace $l(\lambda)+\alpha$ by $\alpha^{-1}(l(\lambda)+\alpha)$ and $m(\lambda)+\beta$ by $\alpha(m(\lambda)+\beta)$, we see 
we may assume $\alpha=1, \beta=-1$. Then equating terms of degree one, we see that $0=l(\lambda)-m(\lambda)$. Equating terms of degree two, we get $\sum \alpha_{i j} \lambda_{i} \lambda_{j}=l(\lambda) m(\lambda)=l(\lambda)^{2}$. I.e. we have a nonzero linear functional $l$ on $\mathfrak{B}$ such that $(v, v)=l(v)^{2}$ for all $v \in \mathfrak{B}$. If we linearize this, we get $(u, v)=l(u) l(v)$ for all $u, v \in \mathfrak{B}$. Now $\operatorname{dim} \mathfrak{B} \geqq 2$, so $l$ has a nonzero kernel. Choose $0 \neq u \in \mathfrak{B}$ so that $l(u)=0$. Then $(u, v)=0$ for all $v \in \mathfrak{B}$, in contradiction to the nondegeneracy of the form.

Proposition 3. If $\mathfrak{\Im}=\Phi 1 \oplus \mathfrak{B}$ is the Jordan algebra of $\mathfrak{B}(\mathfrak{B}$ as in Proposition 2$)$, then $I(\Im)$ is an irreducible algebraic set.

Proof. Since $\mathfrak{I}$ has degree 2 , any idempotent not equal to 0 or 1 is primitive. It is easy to see that $\alpha 1+v(\alpha \in \Phi, v \in \mathfrak{B})$ is a primitive idempotent if and only if $\alpha=\frac{1}{2}$ and $(2 v, 2 v)=1$. Thus the primitive idempotents of $\mathfrak{\Im}$ are exactly the elements of the form $\frac{1}{2}(1+v)$, where $(v, v)=1$. It is thus clear that $I(\Im)=\left\{\alpha 1+v \mid \alpha=\frac{1}{2}\right.$, $\left.(v, v)=\frac{1}{4}\right\}$ is an algebraic set. Also $v \mapsto \frac{1}{2}(1+v)$ is a polynomial map from $I(\mathfrak{B})$ onto $I(\Im)$. So Proposition 2 implies that $I(\Im)$ is irreducible.

As we have seen, this suffices for our applications. We now proceed to the proof of the theorem. $\mathfrak{I}$ is henceforth a simple Jordan algebra over $\Phi$, an algebraically closed field of characteristic at least $n=\operatorname{deg} \mathfrak{\Im}$. Let $t$ be the generic trace of $\Im$. We will use the following facts, whose proofs appear to make use of the classification of simple algebras.

1. Any complete orthogonal set of primitive idempotents has $n$ elements.

2. The trace of any primitive idempotent is 1 .

3. If $e$ is an idempotent of trace 1 , then $e$ is primitive.

To verify these facts, let $e$ be any idempotent. Choose a complete orthogonal set $\left\{e_{1}, \ldots, e_{m}\right\}$ of primitive idempotents such that $e=e_{1}+\cdots+e_{r}$ and $1-e=$ $e_{r+1}+\cdots+e_{m}$. The classification theory [10, pp. 202-205] shows that if $m=1$, then $\mathfrak{F}=\Phi 1$; if $m=2, \mathfrak{I}=\Phi 1 \oplus \mathfrak{B}$, the algebra of a space with a nondegenerate symmetric bilinear form, and $e_{1}=(1+v) / 2, e_{2}=(1-v) / 2$ for some $v \in \mathfrak{B}$ such that $(v, v)=1$; if $m \geqq 3$ then $\Im$ is a Jordan matrix algebra with $e_{i}$ the diagonal matrix units. In each of these cases the generic minimum polynomial is known [10, pp. 229233]; it has degree $m$, and hence $m=n$ (which shows 1). Also $t\left(e_{i}\right)=1, i=1, \ldots, m$. This shows 2 , if we take $e$ to be primitive, i.e. $e=e_{1}$. Finally, if $t(e)=1$, then $r \equiv 1(\bmod p)$, where $p$ is the characteristic of $\Phi$. So $p \mid r-1$. If $r-1 \neq 0$ then $p \leqq r-1$ $\leqq m-1$, a contradiction to $p \geqq n$.

LEMma. Let $\Im$ be as above and $e=e_{1}, e_{2}, \ldots, e_{n}$ a complete orthogonal set of primitive idempotents. For $\gamma \in \Phi, x \in \mathfrak{\Im}_{1 / 2}(e)$, let $f=\gamma^{2} e+\gamma x+x^{2} .(1-e)$. Then $t(f)=\gamma^{2}+\frac{1}{2} t\left(x^{2}\right)$ and $f^{\cdot 2}=t(f) f$. Moreover, if $t(f) \neq 0$ then $t(f)^{-1} f$ is a primitive idempotent of $\mathfrak{~}$.

Conversely, if $f$ is any primitive idempotent of $\mathfrak{\Im}$ such that $f U_{e} \neq 0$, then there exist $\gamma \in \Phi, x \in \Im_{1 / 2}(e)$ so that $f=\gamma^{2} e+\gamma x+x^{.2} .(1-e)$.

Proof. Let $\oplus \sum_{i \leqq j} \mathfrak{\Im}_{i j}$ be the Peirce decomposition of $\mathfrak{\Im}$ with respect to 
$\left\{e_{1}, \ldots, e_{n}\right\}$. Let $x \in \Im_{1 / 2}(e)$, say $x=\sum_{j>1} u_{1 j}$, where $u_{1 j} \in \Im_{1 j}$. Then $x^{2}=$ $\sum_{i, j>1} u_{1 i} \cdot u_{1 j}=\sum_{1<i<j} 2 u_{1 j} \cdot u_{1 i}+\sum_{1<j} u_{1 j}^{2}$. Now $2 u_{1 j} \cdot u_{1 i} \in \Im_{i j}$ and $u_{1 j}^{2}=\frac{1}{2} t\left(u_{1 j}^{2}\right)\left(e_{1}+e_{j}\right)$; for by [10, p. 202], if $x_{i j} \in \Im_{i j}$ then $x_{i j}^{2}=\alpha\left(e_{i}+e_{j}\right)$, and by taking the trace of both sides we find that $\alpha=\frac{1}{2} t\left(x_{i j}^{2}\right)$. Thus

$$
\begin{aligned}
x^{2} & =\left[\sum_{j>1} \frac{1}{2} t\left(u_{i j}^{2}\right)\right] e_{1}+\sum_{j>1} \frac{1}{2} t\left(u_{i j}^{2}\right) e_{j}+\sum_{1<i<j} 2 u_{1 j} \cdot u_{1 i} \\
t\left(x^{\cdot 2}\right) & =\sum_{j>1} \frac{1}{2} t\left(u_{i j}^{2}\right)+\sum_{j>1} t\left(u_{i j}^{2}\right)+0=\sum_{j>1} t\left(u_{i j}^{2}\right) .
\end{aligned}
$$

Since $x^{.2} \in \Im_{1}(e)+\Im_{0}(e)$, the component of $x^{.2}$ in $\Im_{1}(e)$ is $x^{.2} . e$ and the component of $x^{2}$ in $\Im_{0}(e)$ is $x^{2} \cdot(1-e)$. Applying this to the above, we find that

$$
\begin{aligned}
x^{\cdot 2} \cdot e=\frac{1}{2} t\left(x^{2}\right) e, & \\
x^{\cdot 2} \cdot(1-e) & =\sum_{j>1} \frac{1}{2} t\left(u_{i j}^{2}\right) e_{j}+\sum_{1<i<j} 2 u_{1 j} \cdot u_{1 i}, \\
\left(x^{2} \cdot(1-e)\right) \cdot x & =\sum_{j>1} \frac{1}{4} t\left(u_{i j}^{2}\right) u_{1 j}+\sum_{1<i<j}\left[2\left(u_{1 j} \cdot u_{1 i}\right) \cdot u_{1 j}+2\left(u_{1 j} \cdot u_{1 i}\right) \cdot u_{1 i}\right], \\
& =\sum_{j>1} \frac{1}{4} t\left(u_{i j}^{2}\right) u_{1 j}+\sum_{1<i<j}\left[u_{1 j}^{2} \cdot u_{1 i}+u_{i i}^{2} \cdot u_{1 j}\right] .
\end{aligned}
$$

Here we have used a well-known identity [10, identity PD 10, p. 121] which says that $\left(x_{i j} \cdot y_{i j}\right) \cdot z_{j k}=\left(x_{i j} \cdot z_{j k}\right) \cdot y_{i j}+\left(y_{i j}, z_{j k}\right) \cdot x_{i j}$, where the subscripts indicate the Peirce spaces to which the elements belong; in this application we take $j$ to be $1, i$ to be $j, k$ to be $i, x_{i j}=y_{i j}$ to be $u_{1 j}$ in the term $2\left(u_{1 j} \cdot u_{1 i}\right) \cdot u_{1 j}$, and treat the term $2\left(u_{1 j} \cdot u_{1 i}\right) \cdot u_{1 i}$ similarly. Thus

$$
\begin{aligned}
\left(x^{2} \cdot(1-e)\right) \cdot x & =\sum_{j>1} \frac{1}{4} t\left(u_{i j}^{2}\right) u_{1 j}+\sum_{i \neq j} u_{i i}^{2} \cdot u_{1 j} \\
& =\sum_{j>1} \frac{1}{4} t\left(u_{i j}^{2}\right) u_{1 j}+\sum_{i \neq j} \frac{1}{2} t\left(u_{i i}^{2}\right)\left(e_{1}+e_{i}\right) \cdot u_{1 j} \\
& =\sum_{j>1} \frac{1}{4} t\left(u_{i j}^{2}\right) u_{1 j}+\sum_{i \neq j} \frac{1}{4} t\left(u_{i i}^{2}\right) u_{1 j} \\
& =\sum_{j>1} \frac{1}{4} t\left(u_{i j}^{2}\right) u_{1 j}+\sum_{j>1} \sum_{1<i \neq j} \frac{1}{4} t\left(u_{i i}^{2}\right) u_{1 j} \\
& =\sum_{j>1} \sum_{i>1} \frac{1}{4} t\left(u_{1 i}^{2}\right) u_{1 j}=\frac{1}{4} \sum_{i>1} t\left(u_{i i}^{2}\right) \sum_{j>1} u_{1 j}, \\
& \left(x^{2} \cdot(1-e)\right) \cdot x=\frac{1}{4} t\left(x^{2}\right) x .
\end{aligned}
$$

Using equation (1), we find that

$$
\left(x^{2} \cdot e\right) \cdot x=\frac{1}{2} t\left(x^{2}\right) e \cdot x=\frac{1}{4} t\left(x^{2}\right) x .
$$

Adding this equation to (2), we obtain

$$
\begin{gathered}
x^{.3}=\left(x^{.2}\right) x=2 \cdot \frac{1}{4} \cdot\left(t\left(x^{\cdot 2}\right) x,\right. \\
x^{.3}=\frac{1}{2} t\left(x^{.2}\right) x .
\end{gathered}
$$


Using (3), we find that

$$
x^{.4}=x^{.3} \cdot x=\frac{1}{2} t\left(x^{.2}\right) x^{.2}=\frac{1}{2} t\left(x^{.2}\right) x^{2} \cdot e+\frac{1}{2} t\left(x^{.2}\right) \cdot\left(x^{2} \cdot(1-e)\right) .
$$

Also, since $\Im_{0}(e) . \Im_{1}(e)=0$,

$$
x^{.4}=x^{.2} \cdot x^{.2}=\left(x^{2} \cdot e+x^{.2} \cdot(1-e)\right)^{.2}=\left(x^{2} \cdot e\right)^{.2}+\left(x^{.2} \cdot(1-e)\right)^{.2} .
$$

We equate terms in $\Im_{0}(e)$ to obtain

$$
\left(x^{2} \cdot(1-e)\right)^{2}=\frac{1}{2} t\left(x^{\cdot 2}\right) x^{2} \cdot(1-e) .
$$

Now if $f$ is as in the statement of the Lemma, then

$$
\begin{aligned}
f^{.2} & =\gamma^{4} e+\gamma^{2} x^{.2}+\left(x^{2} \cdot(1-e)\right)^{.2}+2 \gamma^{3} e . x+2 \gamma x \cdot\left(x^{2} \cdot(1-e)\right)+2 \cdot 0 \\
& =\gamma^{4} e+\gamma^{2} x^{.2} \cdot e+\gamma^{2} x^{.2} \cdot(1-e)+\frac{1}{2} t\left(x^{.2}\right) x^{\cdot 2} \cdot(1-e)+\gamma^{3} x+2 \gamma \frac{1}{4} t\left(x^{2}\right) x ;
\end{aligned}
$$

here we have used (2) and (4). We apply (1) and collect terms:

$$
f^{.2}=\gamma^{2}\left[\gamma^{2}+\frac{1}{2} t\left(x^{2}\right)\right] e+\left[\gamma^{2}+\frac{1}{2} t\left(x^{.2}\right)\right] x^{.2} \cdot(1-e)+\gamma\left[\gamma^{2}+\frac{1}{2} t\left(x^{.2}\right)\right] x .
$$

Now $t(f)=\gamma^{2}+t\left(x^{.2} .(1-e)\right)=\gamma^{2}+t\left(x^{2}\right)-t\left(x^{2} \cdot e\right)=\gamma^{2}+t\left(x^{2}\right)-\frac{1}{2} t\left(x^{2}\right)=\gamma^{2}+\frac{1}{2} t\left(x^{2}\right)$. I.e. $f^{.2}=t(f) f$. Also, if $t(f) \neq 0$ then we multiply by $t(f)^{-2}$ to get $\left[t(f)^{-1} f\right]^{\cdot 2}=$ $t(f)^{-1} f$. Moreover, $t\left(t(f)^{-1} f\right)=1$, and so $t(f)^{-1} f$ is a primitive idempotent.

We now prove the converse. Let $f$ be a primitive idempotent; suppose $f=f_{0}+$ $f_{1 / 2}+f_{1}$, where $f_{i} \in \Im_{i}(e)$. The hypothesis $f U_{e} \neq 0$ means that $f_{1}=\alpha e, 0 \neq \alpha \in \Phi$. Since $\Phi$ is algebraically closed, we can choose $\gamma \in \Phi$ so that $\gamma^{2}=\alpha$.

Since $f$ is an idempotent, $f_{0}+f_{1 / 2}+f_{1}=\left(f_{0}+f_{1 / 2}+f_{1}\right)^{\cdot 2}=f_{\mathrm{o}}^{2}+f_{\mathrm{i} / 2}^{2}+f_{\mathrm{i}}^{2}+2 f_{0} \cdot f_{1 / 2}+$ $2 f_{1 / 2} \cdot f_{1}+2 \cdot 0$. We equate terms in the various Peirce spaces:

$$
\begin{aligned}
& f_{0}=f_{\dot{0}}^{2}+f_{\mathrm{i} / 2}^{2} \cdot(1-e), \\
& f_{1}=f_{\mathrm{i}}^{2}+f_{\mathrm{i} / 2}^{2} . e .
\end{aligned}
$$

Since $f_{1}=\gamma^{2} e$, we have

$$
\gamma^{2} e=\gamma^{4} e+f_{i / 2}^{2} . e .
$$

Now by (1), $t\left(f_{\mathrm{i} / 2}^{2} \cdot e\right)=\frac{1}{2} t\left(f_{\mathrm{i} / 2}^{2}\right)$. Hence taking traces we find

$$
\frac{1}{2} t\left(f_{i / 2}^{2}\right)=\gamma^{2}-\gamma^{4}
$$

We equate the terms in $\Im_{1 / 2}(e)$ of the original equation and get

$$
\begin{gathered}
2 f_{0} \cdot f_{1 / 2}+2 f_{1} \cdot f_{1 / 2}=f_{1 / 2}, \quad 2 f_{0} \cdot f_{1 / 2}+\gamma^{2} f_{1 / 2}=f_{1 / 2}, \\
2 f_{0} \cdot f_{1 / 2}=\left(1-\gamma^{2}\right) f_{1 / 2} .
\end{gathered}
$$

We apply another well-known identity [10, identity PD 5, p. 121], which says $\left(x_{i j} \cdot y_{i j}\right) \cdot z_{j j}=\left[\left(x_{i j} \cdot z_{j j}\right) \cdot y_{i j}+\left(y_{i j} \cdot z_{j j}\right) \cdot x_{i j}\right] e_{j}$. Here we take $e_{i}$ to be $e, e_{j}$ to be $1-e, x_{i j}$ to be $f_{1 / 2}$ and $z_{j j}$ to be $f_{0}$. We get

$$
f_{\mathrm{i} / 2}^{2} \cdot f_{0}=\left(2\left(f_{0} \cdot f_{1 / 2}\right) \cdot f_{1 / 2}\right) \cdot(1-e)=\left(1-\gamma^{2}\right) f_{\mathrm{i} / 2}^{2} \cdot(1-e)
$$


by (7). So $\left(f_{\mathrm{i} / 2}^{2} \cdot(1-e)\right) \cdot f_{0}=\left(1-\gamma^{2}\right) f_{\mathrm{i} / 2}^{2} \cdot(1-e)$, since $\Im_{1}(e) \cdot \Im_{0}(e)=0$. This together with (5) implies

$$
\begin{aligned}
{\left[f_{0}-\gamma^{-2} f_{\mathrm{i} / 2}^{2} \cdot(1-e)\right] \cdot f_{0} } & =f_{0}^{2}-\gamma^{-2}\left(1-\gamma^{2}\right) f_{i / 2}^{2} \cdot(1-e) \\
& =f_{0}-f_{\mathrm{i} / 2}^{2} \cdot(1-e)-\gamma^{-2} f_{\mathrm{i} / 2}^{2} \cdot(1-e)+f_{\mathrm{i} / 2}^{2} \cdot(1-e) \\
& =f_{0}-\gamma^{-2} f_{\mathrm{i} / 2}^{2} \cdot(1-e) . \\
{\left[f_{0}-\gamma^{-2} f_{\mathrm{i} / 2}^{2} \cdot(1-e)\right] \cdot f_{1 / 2} } & =f_{0} \cdot f_{1 / 2}-\gamma^{-2}\left[f_{\mathrm{i} / 2}^{2} \cdot(1-e)\right] \cdot f_{1 / 2} \\
& =\frac{1}{2}\left(1-\gamma^{2}\right) f_{1 / 2}-\gamma^{-2} \frac{1}{4} t\left(f_{\mathrm{i} / 2}^{2}\right) f_{1 / 2} \quad \text { by }(2) \text { and }(7) \\
& =\frac{1}{2}\left(1-\gamma^{2}\right) f_{1 / 2}-\gamma^{-2} \frac{1}{2}\left(\gamma^{2}-\gamma^{4}\right) f_{1 / 2} \quad \text { by }(6) \\
& =\frac{1}{2}\left(1-\gamma^{2}\right) f_{1 / 2}-\frac{1}{2}\left(1-\gamma^{2}\right) f_{1 / 2}=0 .
\end{aligned}
$$

Furthermore, since $\Im_{1}(e) \cdot \Im_{0}(e)=0$, we have

$$
\left[f_{0}-\gamma^{-2} f_{\mathrm{i} / 2}^{2} \cdot(1-e)\right] . f=f_{0}-\gamma^{-2} f_{\mathrm{i} / 2}^{2} \cdot(1-e),
$$

which means that $f_{0}-\gamma^{-2} f_{i_{/ 2}}^{2} .(1-e) \in \Im_{1}(f)=\Phi f$ (recall $f$ is primitive). Say $f_{0}-\gamma^{-2} f_{\mathrm{i} / 2}^{2} .(1-e)=\beta f=\beta f_{1}+\beta f_{1 / 2}+\beta f_{0}$. Since $f_{0}-\gamma^{-2} f_{i / 2}^{2} \cdot(1-e) \in \Im_{0}(e)$ and $f_{1} \neq 0$, we see by equating terms that $\beta=0$. So $f_{0}=\gamma^{-2} f_{i / 2}^{2}$. $(1-e)$. If $x=\gamma^{-1} f_{1 / 2}$ then clearly $f=\gamma^{2} e+\gamma x+x^{2} \cdot(1-e)$, as desired.

Before giving the proof of the theorem, we make the following observation. Let $\mathfrak{B}$ be a vector space and $U$ a subset of $\mathfrak{B}$. Suppose $U$ is the union of a family $\left\{I_{\alpha}\right\}$ of irreducible relatively (in $U$ ) open sets, any two of which have an element in common. We claim that $U$ is irreducible. Choose a fixed $I_{0}$ in the family. We show that $I_{0}$ is dense in $U$; this will be sufficient, for then $\bar{I}_{0}=\bar{U}$ is irreducible, and so $U$ is irreducible. Now let $W$ be a nonempty open subset of $U$. Then for some $I_{\alpha}, W \cap I_{\alpha} \neq \varnothing$. Also $I_{0} \cap I_{\alpha} \neq \varnothing$. Thus $W \cap I_{\alpha}$ and $I_{0} \cap I_{\alpha}$ are nonempty relatively open subsets of $I_{\alpha}$, which is irreducible. So $I_{0} \cap I_{\alpha} \cap W \neq \varnothing$, which means in particular that $I_{0} \cap W \neq \varnothing$. This shows that $I_{0}$ is dense in $U$.

Proof of Theorem. We choose a complete orthogonal set of primitive idempotents $\left\{e_{1}, \ldots, e_{n}\right\}$ for $\Im$ and apply Proposition 2 to the vector space $\mathfrak{B}_{i}=\Im_{1}\left(e_{i}\right)+$ $\Im_{1 / 2}\left(e_{i}\right)$ for each $i$, where the bilinear form is given by

$$
\left(\gamma e_{i}+x, \gamma e_{i}+x\right)=\gamma^{2}+\frac{1}{2} t\left(x^{2}\right) .
$$

This is clearly a nondegenerate form since $t$ is nondegenerate on $\mathfrak{\Im}_{1 / 2}\left(e_{i}\right)$. The above lemma says that the polynomial function from $I\left(\mathfrak{B}_{i}\right)$ to $\Im$ defined by $\gamma e_{i}+x \rightarrow \gamma^{2} e_{i}+\gamma x+x^{2} .\left(1-e_{i}\right)$ maps $I\left(\mathfrak{B}_{i}\right)$ onto a set of primitive idempotents of $\Im$ containing the set $I_{i}$ of all primitive idempotents $f$ of $\Im$ such that $f U_{e_{i}} \neq 0$ (since $t\left(\gamma^{2} e_{i}+\gamma x+x^{2} \cdot\left(1-e_{i}\right)\right)=1$ in this case, the image set consists of idempotents). This means that $I_{i}$ is an irreducible set. For $I_{i}$ is an open, hence dense, subset of a polynomial image of $I\left(\mathfrak{B}_{i}\right)$, which is irreducible by Proposition 2. If $i \neq j$, then $I_{i} \cap I_{j} \neq \varnothing$. Indeed, $e_{i}$ and $e_{j}$ are connected idempotents. Choose $a \in \mathfrak{\Im}_{i j}$ so that 
$a^{2}=e_{i}+e_{j}$. Then $\frac{1}{2}\left(e_{i}+e_{j}+a\right)$ is a primitive idempotent in $I_{i} \cap I_{j}$ :

$$
\begin{aligned}
{\left[\frac{1}{2}\left(e_{i}+e_{j}+a\right)\right]^{2} } & =\frac{1}{4}\left(e_{i}^{2}+e_{j}^{2}+a^{2}+a+a\right)=\frac{1}{4}\left(2 e_{i}+2 e_{j}+2 a\right) \\
& =\frac{1}{2}\left(e_{i}+e_{j}+a\right), \\
t\left(\frac{1}{2}\left(e_{i}+e_{j}+a\right)\right) & =\frac{1}{2}(1+1+0)=1, \\
\frac{1}{2}\left(e_{i}+e_{j}+a\right) U_{e_{i}} & =\frac{1}{2} e_{i} \neq 0, \\
\frac{1}{2}\left(e_{i}+e_{j}+a\right) U_{e_{j}} & =\frac{1}{2} e_{j} \neq 0 .
\end{aligned}
$$

Also every primitive idempotent $f$ is in some $I_{i}$. For if $f U_{e_{i}}=\alpha_{i} e_{i}$ then $1=t(f)=$ $\sum_{i} \alpha_{i}$, so not all $\alpha_{i}$ are zero. We conclude that $I(\Im)=\bigcup_{i=1}^{n} I_{i}$. This implies that $I(\Im)$ is irreducible, by the above remarks. $I(\Im)$ is certainly closed, since the conditions $x^{2}=x, t(x)=1$ are polynomial conditions.

\section{REFERENCES}

1. Séminaire "Sophus Lie" de l'école normale supérieure 1954/55, Secrétariat mathématique, Paris, 1955. MR 17, 384.

2. C. Chevalley, Théorie des groupes de Lie. Vols. II, III, Actualités Sci. Indust., nos. 1152, 1226, Hermann, Paris, 1951, 1955. MR 14, 448; MR 16, 901.

3. S. R. Gordon, On the automorphism group of a semisimple Jordan algebra of characteristic zero, Bull. Amer. Math. Soc. 75 (1969), 499-504. MR 39 \#4239.

4. K.-H. Helwig, Über Automorphismen und Derivationen von Jordan-Algebren, Nederl. Akad. Wetensch. Proc. Ser. A 70=Indag. Math. 29 (1967), 381-394. MR 37 \#1422.

5. —- Halbeinfache Reele Jordan-Algebren, Habilitationsschrift, München, 1967.

6. N. Jacobson, Derivation algebras and multiplication algebras of semi-simple Jordan algebras, Ann. of Math. (2) 50 (1949), 866-874. MR 11, 76.

7. - Exceptional Lie algebras, Yale University, New Haven, Conn., 1957 (mimeographed notes).

8. - Lie algebras, Interscience Tracts in Pure and Appl. Math., no. 10, Interscience, New York, 1962. MR 26 \#1345.

9.,- A note on automorphisms of Lie algebras, Pacific J. Math. 12 (1962), 303-315. MR 26 \#6222.

10. — Structure and representations of Jordan algebras, Amer. Math. Soc. Colloq. Publ., vol. 39, Amer. Math. Soc., Providence, R. I., 1969.

11. M. Koecher, Imbedding of Jordan algebras into Lie algebras. I, Amer. J. Math. 89 (1967), 787-815. MR 35 \#5480.

12. —_ On Lie algebras defined by Jordan algebras, Århus Universitet, 1967.

13. J.-P. Serre, Algèbres de Lie semi-simples complexes, Benjamin, New York, 1966. MR 35 \#6721.

14. D. A. Smith, Chevalley bases for Lie modules, Trans. Amer. Math. Soc. 115 (1965), 283-299. MR 33 \#4191.

15. R. Steinberg, Lectures on Chevalley groups, Yale University, New Haven, Conn., 1967 (mimeographed notes).

YALE UNIVERSITY,

New Haven, Connecticut 06250 